\title{
Interactions between invertebrate and mycotoxin-producing fungi
}

\author{
Dissertation \\ to obtain the $\mathrm{Ph}$. D. degree \\ in the International Ph. D. Program for Agricultural Sciences in Göttingen (IPAG) \\ at the Faculty of Agricultural Sciences, \\ Georg-August-University Göttingen, Germany
}

Presented by

Yang Xu

Born in China

Göttingen, 05.2018 
D7 (this is the number of our faculty it's important for the university library)

1. Name of supervisor: Prof. Dr. Petr Karlovsky

2. Name of co-supervisor: Prof. Dr. Stefan Vidal

Date of dissertation: 07.09.2018 


\section{Contents}

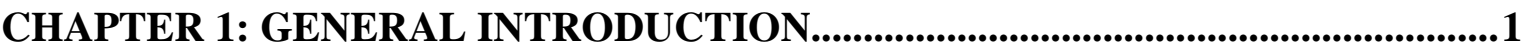

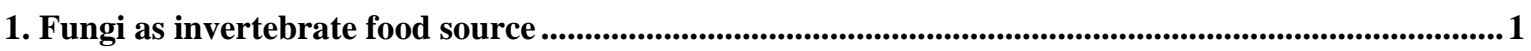

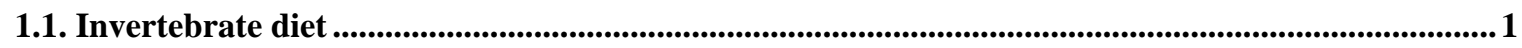

1.2. Effect of predation on fungal biomass ............................................................................................................... 1

2. Mutualism between fungus and invertebrate......................................................................................................2

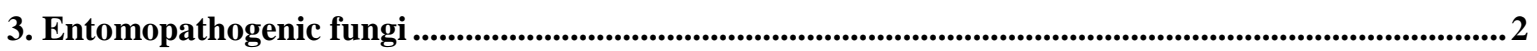

4. Fungi can repel invertebrates .................................................................................................................................

5. Animals as vectors for fungal spores ......................................................................................................................

6. Invertebrate food preference ............................................................................................................................

7. Fungal defense against predation..............................................................................................................

8. Molecules mediates interactions between fungi and invertebrates ...........................................................5

8.1. Secondary metabolites.............................................................................................................................5

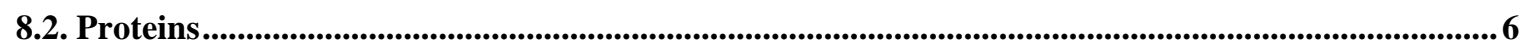

9. Methods used to investigate insect-fungal interactions .....................................................................................

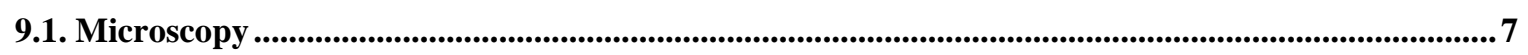

9.2. Food preference and fitness of animals............................................................................................

9.3. Defense analysis of grazed mycelium .....................................................................................................

9.4. Analysis of microorganisms associated with invertebrates ........................................................................

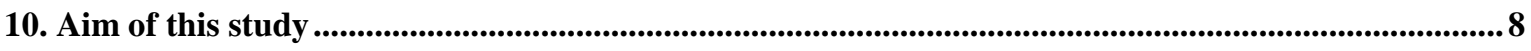

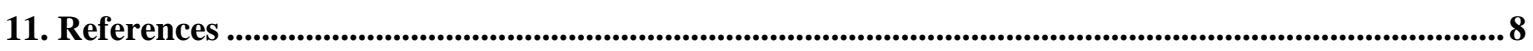

CHAPTER 2: EFFECT OF ANIMAL PREDATION ON THE BIOMASS OF

MYCOTOXIN-PRODUCING FUNGI...................................................................16

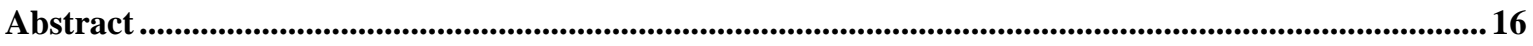




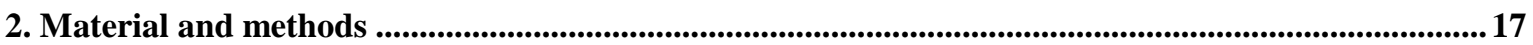

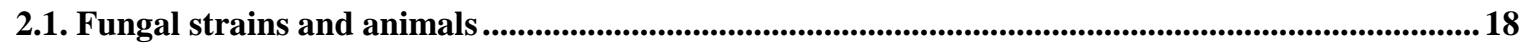

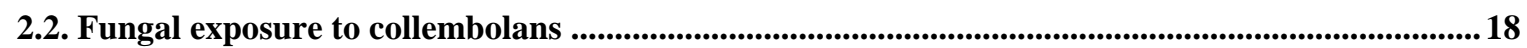

2.3. Preparation of DNA standards for the determination of A. ochraceus, A. nidulans and $P$. viridicatum biomass

2.4. DNA extraction of grazed and non-grazed fungal mycelia ................................................................ 19

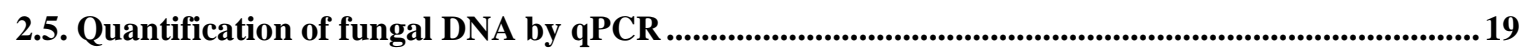

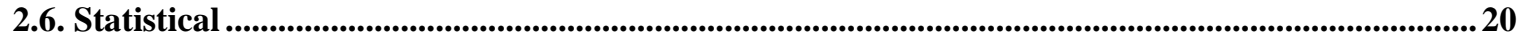

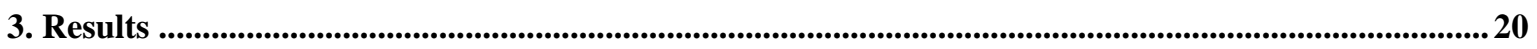

3.1. Standard curve of each fungal strain for real-time PCR ............................................................20

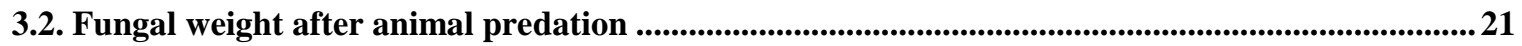

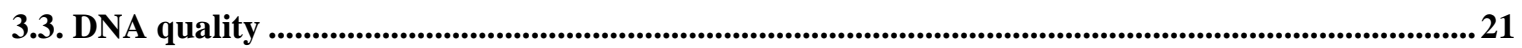

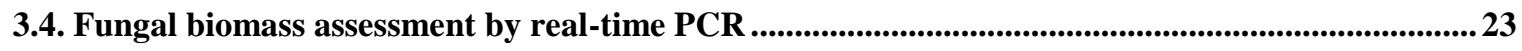

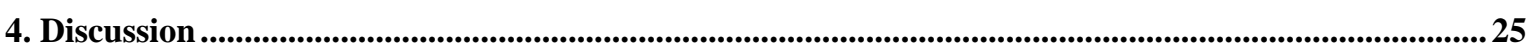

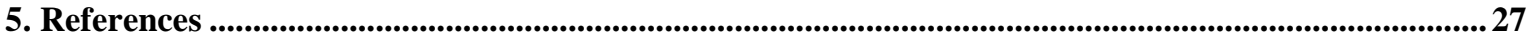

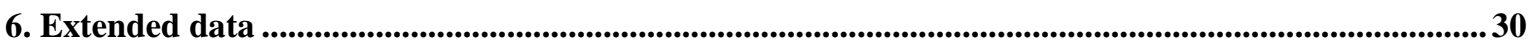

CHAPTER 3: INVESTIGATION OF INTERACTIONS BETWEEN FUSARIUM GRAMINEARUM, FUSARIUM VERTICILLIOIDES AND FOLSOMIA CANDIDA

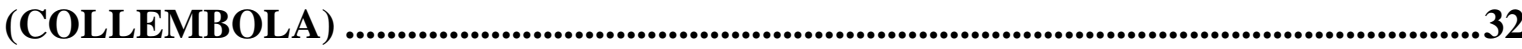

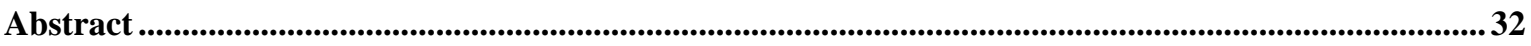

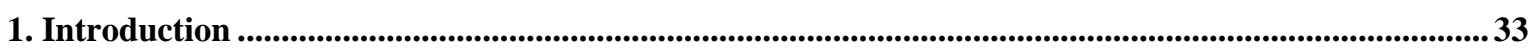

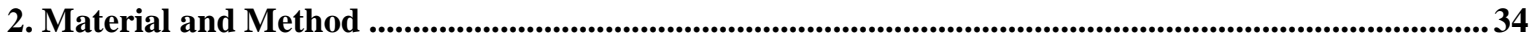

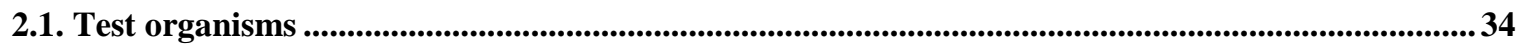

2.2. Food preference of $F$. candida ......................................................................................................3 


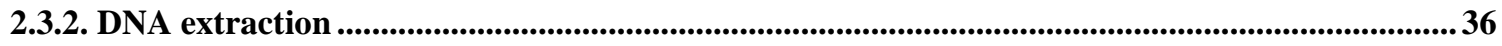

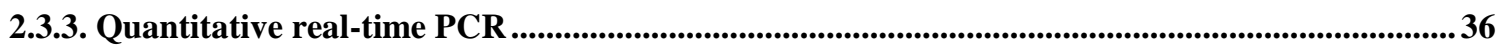

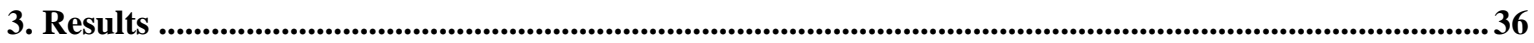

3.1. F. candida food preference between $F$. verticillioides and $F$. graminearum ...................................3.36

3.2. Food choice of $F$. candida offered $F$. verticillioides and $F$. graminearum mutants impared in secondary metabolite synthesis.

3.3. Food preference between $F$. graminearum, $F$. verticillioides, and their mixed culture. 38

3.4. Comparison between food selection and food preference 39

4. Discussion 39

5. References 41

\section{CHAPTER 4: NONTOXIC NAPHTHOPYRONE PIGMENT PROTECTS}

FILAMENTOUS FUNGI FROM A WIDE RANGE OF ANIMAL PREDATORS...44

1. Main text

2. Materials and Methods 53

2.1. Animals .53

2.2. Fungal strains. 53

2.3. Purification of aurofusarin 54

2.4. Exposure of fungi to predation 54

2.5. Transcriptome analysis by RNA sequencing (RNAseq) .54

2.6. Transcription of genes of aurofusarin biosynthetic pathway after predation...............................56

2.7. Food preference of fungivorous arthropods .56

2.8. Effect of aurofusarin on the fitness of fungivorous arthropods 57 


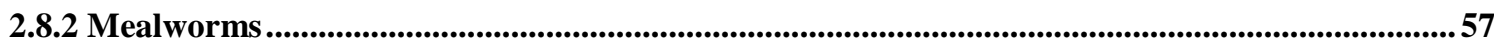

2.9. Toxicity of aurofusarin to insect cells in tissue culture ................................................................58

2.10. Determination of aurofusarin and viomellein by HPLC ...............................................................58

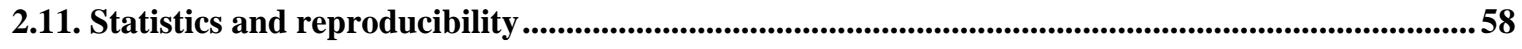

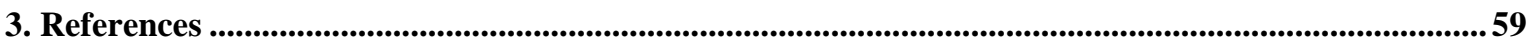

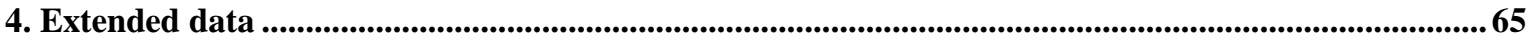

CHAPTER 5: GENERAL DISCUSSION ..................................................................

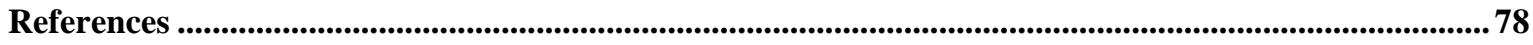

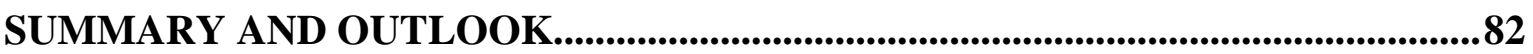

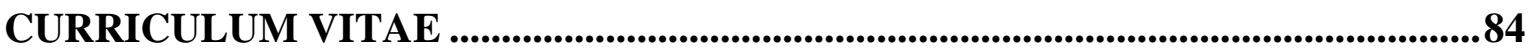

PUBLICATIONS \& WORKSHOPS ........................................................................85

ACKNOWLEDGMENT $\ldots \ldots \ldots \ldots \ldots \ldots \ldots \ldots \ldots \ldots \ldots \ldots \ldots \ldots \ldots \ldots \ldots \ldots \ldots \ldots \ldots \ldots \ldots \ldots \ldots \ldots \ldots \ldots \ldots \ldots \ldots \ldots \ldots \ldots \ldots \ldots . \ldots . \ldots \ldots 7$

STATUTORY DECLARATION ....................................................................................8 


\section{Important abbreviations}

$F$. graminearum $\triangle$ AUR: $F$. graminearum aurofusarin deficient stain

$F$. graminearum $\triangle \mathrm{DON}: F$. graminearum deoxynivalenol deficient stain

$F$. graminearum $\triangle \mathrm{ZEA}: F$. graminearum zearalenone deficient stain

F. graminearum WT: F. graminearum Wild Type

RT-PCR: Reverse transcription polymerase chain reaction

HPLC-DAD: High-performance liquid chromatography with diode-array detection

HPLC-MS: High-performance liquid chromatography-mass spectrometry

RNAseq: Whole transcriptome shotgun sequencing

SSCPs: Small secreted cysteine-rich proteins

GAPDH: Glyceraldehyde 3-phosphate dehydrogenase

EFIA: Elongation factor 1-alpha 


\section{Chapter 1: General Introduction}

Numerous small animals (arthropods, oligochaetes, mollusks and nematodes) and fungal species coexist in nature (Gange \& Brown, 2002). Predatory, mutualistic, parasitic and pathogenic fungi-invertebrate relationships exist that have been extensively described (Renker et al., 2005; Maraun et al., 2003; Lilleskov \& Bruns, 2005).

\section{Fungi as invertebrate food source}

\subsection{Invertebrate diet}

Invertebrates such as collembolans, mites, and nematodes, play an important role in decomposer networks by consuming and transforming organic matter in soil (Pflug \& Wolters, 2000). Fungi represent as a high-quality food source. They contain ergosterol, steroids, proteins, vitamins and lipids. Non-entomopathogenic fungi can provide rich nutrient sources for a wide variety of insects and nematodes. Fungivorous animals, such as arachnids, acari, collembolans and nematodes mainly rely on fungal fruiting bodies and spores as a food source (Schigel, 2012). Invertebrates usually live in nutrient-poor environments. Consuming nutrient-rich fungi provides a more balanced diet (Fano et al., 1982).

\subsection{Effect of predation on fungal biomass}

Generally, the comparatively low number of animals in the field cannot reduce the population of fungi but rather facilitate its proliferation. However, intensive grazing by invertebrates can decrease the fungal population, though the effect species-specific (Crowther et al., 2012a).

Ciid beetles (Octotemus glabriculus and Cis boleti) can reduce fungal reproductive potebtial by $30-58 \%$, while, in the short range, they reduce the amount of fungal mycelium (Guevara et al., 1999). Wolfarth et al. (2013) reported that the nematode Aphelenchoides saprophilus and the collembolan Folsomia candida reduce the biomass of Fusarium culmorum. Meloidogyne spp., by contrast, enhance the severity of Fusarium wilt in cotton root (Hasan, 1993).

Generally, the enchytraeid Enchytraeus crypticus and the oribatid mite Euzetes globulus do not affect fungal growth in natural sites. The collembolans $F$. candida and nematode 
Panagrellus redivivus, however, affect fungal community structure. The woodlice Oniscus asellus significantly affects fungal species composition in wood decomposer communities (Crowther et al., 2011a, b).

\section{Mutualism between fungus and invertebrate}

Invertebrate hosts cannot survive without their microbial symbionts. A wide range of fungi and invertebrates developed long-term, intimate, and mutually beneficial relations (Dowd, 1992; Engel \& Moran, 2013). The invertebrate benefits from the fungus in terms of predigestion of food and detoxification of toxins.

Higher termites (e.g. Atta cepbalotes) are reportedly using garden fungal cultures as food source (Mueller et al., 2002). Dowd (1992) revealed that fungi could produce cellulolytic enzymes that facilitate feeding on wood to beetles. Cochliobolus lunatus, isolated from Palythoa haddoni, was able to produce resorcylic acid lactones that defend the host against pathogenic fungi (Liu et al., 2014). Aspergillus unguis, isolated from Sinularia sp., produce phenolic and triterpenoid compounds that protect the host against the pathogenic fungi Candida albicans and Aspergillus flavus (Putri et al., 2015). In addition, saprophytic fungi can detoxify allelochemicals in wood, decompose lignin, and enhance nitrogen availability (Dowd, 1992).

\section{Entomopathogenic fungi}

The potential of application in biological pest management has initiated many studies of entomopathogenic fungi. Around 1,000 fungal species are reportedly able to infect or kill invertebrates, among them fungi from various divisions such as Ascomycota, Basidiomycota, Entomophthoromycota, Microsporidia and Chytridiomycota (Araujo \& Hughes, 2016; Vega et al., 2012).

Fungi are able to attack invertebrates and cause death through various mechanisms (Mora et al., 2017). Smittium morbosum invade mosquito larvae through the gut and inhibit moulting of the cuticula, which eventually leads to the death of the animal. It invades the ovaries of black flies (Simuliidae). The fungus Ophiocordyceps even infects the brain of the ant Camponotus. As a result, the ant loses control over its behavior by choosing a place 
to die that is also suitable to the fungus, for example the underside of leaves (Araujo et al., 2015; Araujo \& Hughes, 2016).

\section{Fungi can repel invertebrates}

Various fungal-invertebrate interactions have been studied. However, fungi that are not attractive to fungal-feeding insects are yet to be investigated. For example, Jorgensen et al. (2005) found 33 different fungal species in soil. Eight of them were detected on the surface of the collembolan Protaphorura armata, but only one was found in its gut. This suggests that the majority of fungi may repel collembolans successfully. The potentially involved mechanisms have not been explored yet.

\section{Animals as vectors for fungal spores}

Usually, invertebrates are considered as important vectors for fungal dispersal (Lilleskov et al., 2005; Jacobsen et al., 2017). Invertebrates have been found related to a variety of fungal species (Renker et al., 2005; Hu et al., 2015), which raises the possibility that these fungi could potentially be dispersed in the natural ecosystem by their invertebrate host-For example, various fungal spores can be isolated from bodies of bark beetles, which eventually reach their plant host with transportation of beetles (Paine et al., 1997).

Fungal spores occur in the gut, on the surface and in fecal pellets of invertebrates (Klepzig et al., 2001). Some fungal spores are resistant to digestion by invertebrates and are still viable after passing through the gut of invertebrates (Vannier, 1979). Reportedly, fungi can manipulate the animal brain to disperse them to suitable locations (Araujo et al., 2015). Hypogeous fungi only reproduce underground in a closed system and thus soil animals contribute to their spore dispersal.

The presence of Meleodogyne incognita, M. hapla and Heterodera glycines increased the severity of Fusarium wilt infection in tomato, cotton, peas and tobacco (Mai, 1987). Kluth et al. (2002) showed that Aphis fabae, Uroleucon cirsii and Cassida rubiginosa enhance the infection of Puccinia punctuformis on the weed Cirsium arvense. The increased severity might largely be due to the spread of fungal spores by invertebrates.

\section{Invertebrate food preference}


Small animals are considered feed selectively and are attracted to various fungi by different signals that are probably caused by differences in morphology and mycelial chemistry (odor, color, nutrient content, undigested compounds, amongst others). Kaneda \& Kaneko (2004) reported that Folsomia candida prefers mycelium of the fungus Pisolithus tinctorius cultured on MMN agar medium (modified Melin-Norkrans medium) more than if it occurs as mycorrhiza of pine trees. Maraun et al., (2003) reported that invertebrates usually prefer dark pigmented microfungi. Penicillium spp. and Aspergillus spp., however, are exceptions. Occasionally, other pathogenic fungi are a preferred by collembolans (Scheu \& Folger, 2004; Maraun et al. 2003). Interestingly, fungi (such as Aspergillus spp.) are repellent to collembolans when separately cultured, but this phenomenon disappears when they grow together (Scheu et al., 2004a, b; McGonigle, 2007).

Invertebrate grazing causes specific effects on fungal communities (A'Bear et al., 2013; Crowther et al., 2011a, b). Invertebrate selective grazing is not only able to alter fungal community composition but also can enhance fungal diversity (Rotheray et al., 2011). Different food preference likewise facilitate the coexistence of different invertebrates in the ecological niche (Fano et al., 1982).

\section{Fungal defense against predation}

High nutritive value and lack of motility cause fungi to be attractive to predators. However, when threatened by predation, fungi try to defend themselves either by mechanical means, such as crystals (Böllmann et al., 2010), or chemical means, secondary metabolites, peptides and proteins, amongst others (Rohlfs et al., 2007; 2011; Stötefeld et al., 2012; Tayyrov et al., 2018). As a result, they become less palatable and more toxic.

Biosynthesis of additional metabolites costs energy. Therefore, fungi can produce certain potential chemical weapons in specific ecological conditions. Some metabolites that are constitutively produced during fungal growth, can also serve as chemical defense. Others are only expressed or induced in response to antagonism, such as attack by predators or in stress conditions (Rohlfs, 2015).

Fungivory damages fungal tissue during chewing, which probably triggers signal cascades leading to induction of toxic chemicals. For instance, higher fungi synthetize woundactivated precursor compounds which convert to highly toxic products following tissue 
damage (Spiteller, 2008). Until now, many studies have convincingly demonstrated that fungi are capable of displaying an induced chemical response to invertebrate predator attack (Döll et al., 2013; Spiteller, 2008; Rohlfs et al., 2007; 2011).

\section{Molecules mediates interactions between fungi and invertebrates}

The investigation of interaction between fungi and invertebrate has accelerated in recent years. Molecules, including fungal secondary metabolites and proteins, are essential components that mediate these interactions. On one hand they facilitate the location and invasion of the insect body, on the other hand some of them attract and others deter predators.

\subsection{Secondary metabolites}

Fungi emit volatile chemicals emitted that can attract invertebrates. Morath et al. (2012) reported that 1-octen-3-ol, $\alpha$-cadinene, $\beta$-guaiene, isoledene and $\gamma$-patchoulene produced by Trametes can attract fungus-eating beetles. Chokol K and a methyl ester, methyl (Z)-3methyldodec-2-enoate released by Epichloe attracts Botanophila flies. Protaphorura armata and other species are attracted to volatile compounds that have been extracted from their preferred fungal food sources (Bengtsson et al., 1991; Hedlund et al., 1995).

Fungal secondary metabolites can deter small animals. Furocoumarin (neurosporin A) is able to deter the collembolan Sinella curviseta (Zhao et al. 2017). Fungi synthetize pungent or bitter compounds in their fruiting body in attempts to deter fungivores (Spiteller, 2008). Accumulation of calcium oxalate crystals and increased skin thickness reduce the palatability of fungal fruiting bodies (Böllmann et al. 2010). Naphthalene produced by Muscodor vitigenus repels insects effectively (Morath et al., 2012). Grazing by invertebrates induced several secondary metabolites (sterigmatocystin, emericellamide, austinol and dehydroaustinol) in Aspergillus nidulans (Döll et al., 2013).

In addition, secondary metabolites can cause reduce animal fitness if they are present in their diet. Feeding experiments on mutant lines that lack the respective biosynthetic capabilities corroborated this effect. For instance, the crude extract of five Penicillium strains caused weight loss and mortality of the cotton leafworm Spodoptora littoralis (Paterson et al., 1987). Rohlfs et al. (2007) discovered that Dlae Aspergillus nidulans, which is lack of gene in regulation of production of secondary metabolites, increased the 
fitness and reproductive rate of feeding invertebrates compared to the wild type strain. $\Delta P k s P$ Aspergillus fumigatus, whose melanin biosynthesis pathway had been disrupted, appeared to represent a diet of higher quality to collembolans (Scheu and Folger, 2004).

Many secondary metabolites, such as destruxins, cytochalasin D, beauvericin, oosporein, bassianolide, cordycepin, serinocylin A and beauverolides, metabolites possess insecticidal effects (Molnar et al., 2010; Wei et al., 2017).

Some compounds cause no measurable effect on insect fitness, but contribute in a more subtle way. NG-391, a mycotoxin that is similar to fusarins produced by Fusarium spp, accumulates during Metarhizium robertsii infection of Spodoptera exigua. However, it causes no measurable negative effect to the insect (Donzelli et al., 2010). 1, 4benzoquinone oxidoreduetase produced by Beauveria bassiana assist fungal infection by detoxifying benzoquinone containing secretions on the epicuticle of red flour beetle (Tribolium castaneum) (Pedrini, 2015).

The role of secondary metabolites in fungus-invertebrate interactions has been studies in some cases. Our knowledge, however, is still rudimentary and many further studies are needed to increase the insights.

\subsection{Proteins}

The ability of a fungus to distinguish itself from others is an important part of defense. Fungi are able to recognize compatible cells (reproductive process) from non-compatible cells by proteins (Bidard et al., 2013). Certain fungivory-induced proteins can be recognized by the host fungus and thus initiate a defense process.

Fungal proteins are capable of targeting epithelial surfaces in lumen of host digestive tract, which results in inhibiting the host's development or killing it. Ascomycota and Basidiomycota produce hololectin that is reported to help fungi to resist insect attacks. Several fungal proteins are reportedly highly toxic to insect and nematodes (Künzler, 2015; Butschi et al., 2010; Sabotic et al., 2016). Melanizing proteins from several fungal species have been proven to cause insecticidal effect as well (Mora et al., 2017).

Forest soil fungi upregulate the expression of several enzymes after being grazed by soil animals. Among them, enzymes, such as glucosidases, cellulase, $\mathrm{N}$-acetylglucosaminidase, phosphatases and phosphodiesterases, were found (Crowther et al., 2011). Their main function is accelerating the decomposition of organic compounds in soil. The mechanisms that confers to defense of soil invertebrates has not been elucidated yet. 


\section{Methods used to investigate insect-fungal interactions}

\subsection{Microscopy}

Light and scanning electron microscopy can be used to observe the growth of fungi, such as fungal colonisation location as well as adhesion and penetration structure. They are widely used to study fungal infection process (Asensio et al., 2005; Jolly et al., 1993; Gunnarsson, 1988). Böllmann et al. (2010) explored crystal formation of fungal metabolites by using light microscopy.

\subsection{Food preference and fitness of animals}

Food preference is investigated by offering different fungal diet at opposite ends of an arena and recording the number of animals visiting each fungal diet. Fitness is determined by measuring invertebrate body size, reproductive rate, and fecal pellet production, amongst others (Crowther et al., 2011, 2012; Böllmann et al., 2010; Rohlfs et al., 2007; Scheu \& Simmerling, 2004).

\subsection{Defense analysis of grazed mycelium}

Mycelium injury is considered an important procedure to induce the biosynthesis of defense compounds. Specific injury-derived compounds can be identified by comparing mycotoxin patterns in intact to damaged mycelium using liquid chromatography (HPLC) with UV (DAD) or mass spectroscopy (MS) as detectors, or even combinations of them (Rohlfs et al., 2011).

To explore the potential benefits of synthetizing specific mycotoxins or secondary metabolites, genetically modified fungal strains lacking specific biosynthetic can be compared the their respective wild types (Rohlfs et al., 2007; Döll et al., 2013; Zhao et al. 2017). This approach provides us with a more realistic scenario than using pure chemical compounds in bioassays.

\subsection{Analysis of microorganisms associated with invertebrates}

Animal diet composition and food preference can be revealed by analyzing animal gut content (Hopkin et al., 1997). Culture-dependent and molecular methods were generally used. A variety of conditions combined with different culture media (potato dextrose, malt extract), temperature, oxygen levels (aerobic or anaerobic), and light conditions were used to isolate fungal strains from invertebrate guts. This work yielded fungal isolates from agricultural and forestry invertebrates. However, due to the growth bias of each fungal 
species, it is possible that only very small portion of fungi related with each invertebrate species can be isolated using a culture-based method. In the last decades, many molecular methods such as DGGE, RFLP and TFLP have been developed that allow exploring fungal communities in animal guts (Grigorescu et al., 2018).

\section{Aim of this study}

Mycotoxin-producing fungi are common in the environment, which cause crop quality decrease, cereal yield loss, plant and animal wellness damage, economic loss and so on. However, the interaction between these fungi and invertebrates in the field are rarely studied. The goal of this study was to elucidate interactions between selected fungivorous invertebrates and fungi, which would provide us a better understanding of effect of collembolan grazing on mycotoxin-producing fungi, and the fungal secondary metabolites biological function during various invertebrate interact with fungi. A combination of molecular, chemical and ecological methods and biological assays were used in this study.

\section{References}

Araujo, J.P.M., Evans, H.C., Geiser, D.M., Mackay, W.P. and Hughes, D.P. (2015). Unravelling the diversity behind the Ophiocordyceps unilateralis (Ophiocordycipitaceae) complex: Three new species of zombie-ant fungi from the Brazilian Amazon. Phytotaxa. 220: 224-238.

Araujo, J.P.M. and Hughes, D.P. (2016). Diversity of Entomopathogenic Fungi: Which Groups Conquered the Insect Body? Advances in Genetics. 94: 1-39.

Asensio, L., Lopez-Llorca, L.V. and Lopez-Jimenez, J.A. (2015). Use of light, cscanning electron microscopy and bioassays to evaluate parasitism by entomopathogenic fungi of the red scale insect of palms (Phoenicococcus marlatti CkII., 1899). Micron 36: 169-175.

A'Bear, A.D., Murray, W., Webb, R., Boddy, L. and Jones, T.H. (2013). Constrasting effects of elevated temperature and invertebrate grazing regulate multispecies interaction between decomposer fungi. Plos One 8 (10) .

Bengtsson, G., Hedlund, K. and Rundgren, S. (1991). Selective odor perception in the soil collembola Onychiurus armatus. Journal of Chemical Ecology 17: 2113-2125.

Bidard, F., Clave, C. and Saupe, S.J. (2013). The transcriptional response to nonself in the gungus Podospora anserine. G3: Genes, Genomes, Genetics 3: 1015-1030. 
Butschi, A., Titz, A., Wälti M.A., Olieric, V., Paschinger, K., Nöbauer, K., Guo, X.Q., Seeberger, P.H., Wilson, L.B.H., Aebi, M., Hengartner, M.O. and Künzler, M. (2010). Caenorhabditis elegans N-glycan core $\beta$-galactoside confers sensitivity towards nematotoxic fungal galectin CGL2. Plos Pathogens. 6: doi: 10.1371/journal.ppat.1000717.

Böllmann, J., Elmer, M., Wöllecke, J., Raidl, S. and Hüttl, R.F. (2010). Defensive strategies of soil fungi to prevent grazing by Folsomia candida (Collembola). Pedobiologia 53: 107-114.

Crowther, T.W., Boddy, L. and Jones, T.H. (2011a) Outcomes of fungal interactions are determined by soil invertebrate grazers. Ecology Letters 14: 1134-1142.

Crowther, T.W., Jones, T.H. and Boddy, L. (2011b). Species-specific effects of grazing invertebrates on mycelial emergence and growth from woody resources into soil. Fungal Ecology 4: 333-341.

Crowther, T.W., Boddy, L. and Jones, T.H. (2012). Functional and ecological consequences of saprotrophic fungus-grazer interactions. The ISME Journal 6: 1992-2001.

Crowther, T.W. and Donald, A'Bear. (2012). Impacts of grazing soil fauna on decomposer fungi are species-specific and density-dependent. Fungal Ecology 5: 277-281.

De Faria, M.R., Wraight, S.P. (2007). Mycoinsecticides and mycoacaricides: A comprehensive list with wordwide coverage and international classification of formulation types. Biological Control 43: 237-256.

Donzelli, B.G., Krasnoff, S.B., Churchill, A.C., Vandenberg, J.D. and Gibson, D.M. (2010). Identification of a hybrid PKS-NRPS required for the biosynthesis of NG391 in Metarhizium robertsii. Current Genetics 56: 151-162.

Dowd, P.F. (1992). Insect fungal symbionts: a promising source of detoxifying enzymes. Journal of Industrial Microbiology 9: 149-161.

Döll, K., Chatterjee, S., Scheu, S., Karlovsky, P. and Rohlf, M. (2013). Fungal metabolic plasticity and sexual development mediate induced resistance to arthropod fungivory. Proceedings of the Royal Society B: Biological Sciences 280: 20131219. 
Engel, P. and Moran N.A. (2013). The gut microbiota of insects - diversity in structure and function. FEMS Microbiology Reviews 37: 699-735.

Fano, E.A., Rossi, L. and Basset, A. (1982). Fungi in the diet of three benthic invertebrate species. Bolletino di zoologia 49: 99-105.

Gange, A.C. and Brown, V.K. (2002). "Actions and interactions of soil invertebrates and arbuscular mycorrhizal fungi in affecting the structure of plant communities" In: Mycorrhizal Ecology (M. G. A. van der Heijden, G. A. Sanders eds.) Springer, Berlin, Herdelberg, pp 321-344.

Grigorescu, A.S., Renoz, F., Sabri, A., Foray, V., Hance, T. and Thonart, P. (2018). Accessing the hidden microbial diversity of aphids: an illustration of how culturedependent methods can be used to decipher the insect microbiota. Microbial Ecology 75: 1035-1048

Guevara, R., Rayner, A.M. and Reynolds, S. (1999). Effects of fungivory by two specialist ciid beetles (Octotemnus glabriculus and Cis boleti) on the reproductive fitness of their host fungus, Coriolus versicolor. New Phytologist 145: 137-144.

Gunnarsson, S.G.S. (1988). Infection of Schistocerca gregaria by the fungus Metarhizium anispliae: cellular reactions in the integument studied by scanning electron and light microscropy. Journal of Invertebrate Pathology 52: 9-17.

Hasan, A. (1993). "The role of fungi in fungus-nematode interactions" In: Nematode Interactions (M. W. Khan, ed.) Springer, Dordrecht, pp 273-287.

Hedlund, L., Bengtsson, G. and Rundgren, S. (1995). Fungal odour discrimination in two sympatric species of fungivorous collembolans. Functional Ecology 9: 869-875.

Hopkin, S.P. (1997). Biology of the springtails (Insecta, Collembola). Oxford: Oxford University Press.

Hu, X., Li, M. and Chen, H. (2015). Community structure of gut fungi during different developmental stages of Chinese white pine beetle (Dendrotonus armandi). Scientific Reports 5.

Jacobsen, R.M., Kauserud, H., Sverdrup-Thygeson, A., Bjorbaekmo, M.M. and Birkemoe, T. (2017). Wood-inhabiting insects can function as targeted vectors for decomposer fungi. Fungal Ecology 29: 76-84. 
Jolly, J.M., Lappin-Scott, H.M., Anderson, J.M. and Clegg, C.D. (1993). Scanning electron microscopy of the gut microflora of two earthworms: Lumbricus terrestris and Octolasion cyaneum. Microbial Ecology 26: 235-245.

Jorgensen, H.B., Johansson, T., Canbäck, B., Hedlund, K. and Tunlid, A. (2005). Selective foraging of fungi by collembolans oin soil. Biology Letters 1: 243-246.

Kaneda, S. and Kaneko, N. (2004). The feeding preference of a collembolan (Folsomia candida Willem) on ectomycorrhiza (Pisolithus tinctorius (Pers.)) varies with mycelial growth condition and vitality. Applied Soil Ecology 27: 1-5.

Klepzig, K.D., Moser, J.C., Lombardero, F.J., Hofstetter, R.W. 2001. Symbiosis and competition: complex interactions among beetles, fungi and mites. Symbiosis 30: 83-96.

Kluth, S., Kruess, A. and Tscharntke, T. (2002). Insects as vectors of plant pathogens: mutualistic and antagonistic interactions. Oecologia 133: 193-199.

Künzler, M. 2015. Hitting the sweet spot-glycans as targets of fungal defense effector proteins. Molecules 20: 8144-8167.

Leger, R.J.S., Cooper, R.M. and Charnley, A.K. (1985). Cuticle-degrading enzymes of entomopathogenic fungi: cuticle degradation in vitro by enzymes from entomopathogens. Journal of Invertebrate Pathology 47: 167-177.

Lilleskov, E.A. and Bruns, T. (2005). Spore dispersal of a resupinate ectomycorrhizal fungus, Tomentella sublilacina, via soil food webs. Mycologia 97: 762-769.

Liu, Q.A., Shao, C.L., Gu, Y.C., Blum, M., Gan, L.S., Wang, K.L., Chen, M. and Wang C.Y. (2014). Antifouling and fungicidal resorcylic acid lactones from the sea anemone-derived fungus Cochliobolus lunatus. Journal of Agricultural and Food Chemistry 62: 3183-3191.

Mai, W.F. and Abawi G.S. (1987). Interactions among root-knot nematodes and Fusarium wilt fungi on host plants. Annual Review of Phytopathology 25: 317-338.

Maraun, M., Martens, H., Migge, S., Theenhaus, A. and Scheu, S. (2003). Adding to 'the enigma of soil animal diversity': fungal feeders and saprophagous soil invertebrates prefer similar food substrates. European Journal of Soil Biology 39: 85-95. 
McGonigle, T.P. (2007). "Effects of animals grazing on fungi." In: Environmental and Microbial Relationships (C. Kubicek, I. S. Druzhinina) Springer, Berlin, Heidelberg, pp 201-212.

Molnar, I., Gibson, D. and Krasnoff, S. (2010). Secondary metabolites from entomopathogenic hypocrealean fungi. Natural Product Reports 27: 1241-1275.

Mora, M.A.E., Castilho, A.M.C. and Fraga, M.E.F. (2017). Classification and infection mechanism of entomopathogenic fungi. Agricultural Microbiology 84: 1-10.

Morath, S.U., Hung, R. and Bennett, J.W. (2012). Fungal volatile organic compounds: a review with emphasis on their biotechnological potential. Fungal Biology Reviews 26: 73-83.

Mueller, U.G. and Gerardo, N. (2002). Fungus-farming insects: multiple origins and diverse evolutionary histories. Roceedings of the National of the United States of America 99: 15247-15249.

Paine, T.D., Raffa, K.F. and Harrington, T.C. (1997). Interactions among scolytid bark beetles, their associated fungi, and live host conifers. Annual Review of Entomology 42: 179-206.

Paterson, R.P.M., Simmonds, M.S.J. and Blaney, W.M. (1987). Mycopesticidal effects of characterized extracts of Penicillium isolateds and purified secondary metabolites (including mycotoxins) on Drosophila melanogaster and Spodoptora littoralis. Journal of Invertebrate Pathology 50 (2): 124-133.

Pedrini, N., Ortiz-Urquiza. A., Bonnet, C.H., Fan Y., Juarez M.P. and Keyhani, N.O. 2015. Tenebrionid secretions and a fungal benzoquinone oxidoreductase form competing components of an arms race between a host and pathogen. Proceedings of the National Academy of Sciences of the United States of America 112: 3651-3660.

Pflug, A. and Wolters, V. (2002). Collembola communities along a European transect. European Journal of Soil Biology 38: 301-304.

Putri, D.A., Radjasa, O.K. and Pringgenies, D. (2015). Effectiveness of marine fungal symbiont isolated from soft coral sinularia sp. from Panjang island as antifungal. Procedia Environmental Sciences 23: 351-357.

Reboul, J. and Ewbank, J.J. (2016). GPCRs in invertebrate innate immunity. Biochemical Pharmacology 114: 82-87. 
Renker, C., Otto, P., Schneider, K., Zimdars, B., Maraun, M. and Buscot, F. (2005). Oribatid Mites as potential vectors for soil microfungi: study of mite-associated fungal species. Microbial Ecology 50: 518-528.

Rohlfs, M., Albert, M., Keller, N.P. and Kempken, F. (2007). Secondary chemicals protect mould from fungivory. Biology Letters 3: 523-525.

Rohlfs, M. and Churchill, C. (2011). Fungal secondary metabolites as modulators of interactions with insects and other arthropods. Fungal Genetics and Biology 48: $23-34$.

Rohlfs, M. (2015). Fungal secondary metabolite dynamics in fungus-grazer interactions: novel insights and unanswered questions. Frontiers in Microbiology: 5: doi:10.3389/fmicb.2014.00788

Rotheray, T.D., Chancellor, M., Jones, T.H. and Boddy, L. (2011). Grazing by collembolan affects the outcome of interspecific mycelial interactions of cordforming basidiomycetes. Fungal Ecology 4: 42-55.

Sabotic, J., Ohm, R.A. and Künzler, M. (2016). Entomotoxic and nematotoxic lectins and protease inhibitors from fungal fruiting bodies. Applied Microbiology and Biotechnology 100: 91-111.

Scheu, S. and Folger, M. (2004a). Single and mixed diets in Collembola: effects on reproduction and stable isotope fractionation. Functional Ecology 18 (1): 94-102.

Scheu, S. and Simmerling, F. (2004b). Growth and reproduction of fungal feeding Collembola as affected by fungal species, melanin and mixed diet. Oecologia 139: $347-353$.

Schigel, D.S. (2012). Fungivory and host associations of Coleoptera: a bibliography and review of research approaches. 3: 258-272.

Spiteller, P. (2008). Chemical defence strategies of higher fungi. Chemistry. 14: 91009110

Stötefeld, L., Scheu, S. and Rohlfs, M. (2012). Fungal chemical defence alters densitydependent foraging behavior and success in a fungivorous soil arthropod. Ecological Entomology 37: 323-329. 
Tayyrov, A., Schmieder, S.S., Bleuler-Martinez, S., Plaza, D.F. and Künzler, M. (2018). Toxicity of potential fungal defense proteins towards the fungivorous nemetodes Aphelenchus avenae and Bursaphelenchus okinawaensis. Applied and Environmental Microbiology doi: 10.1128/AEM.02051-18

Vannier, G. (1979). Relations trophiques entre la microfaune et la microflore du sol; aspects qualitatifs et quantitatifs. Bolletino di zoologia 46: 343-361.

Vega, F.E., Meyling, N.V., Luangsa-Ard, J.J., Blackwell, M. (2012). "Fungal entomopathogens" In Insect Pathology (F. Vega, H. Kaya, eds.) Academic Press, London, pp 171-220.

Wang, C. and St Leger, R.J. (2007). The MAD1 adhesin of Metarhizium anisopliae links adhesion with blastospore production and virulence to insects, and the MAD2 adhesin enables attachment to plants. Eukaryotic Cell 6: 808-816.

Wei, G., Lai, Y.L., Wang, G.D., Chen, H., Li, F. and Wang, S.B. (2017). Insect pathogenic fungus interacts with the gut microbiota to accelerate mosquito mortality. Proceedings of the National Academy of Sciences of the United States of America 114: 5994-5999.

Wolfarth, F., Schrader, S., Oldenburg, E. and Weinert, J. (2013). Nematode-collembolaninteraction promotest he degradation of Fusarium biomass and deoxynivalenol according to soil texture. Soil Biology and Biochemistry 57: 903-910.

Zhang, J., Jin, K. and Xia, Y.X. (2017). Contributions of $\beta$-tubulin to cellular morphology, sporulation and virulence in the insect-fungal pathogen, Metarhizium acridum. Fungal Genetics and Biology 103: 16-24

Zhao, Y.X., Ding, J.N., Yuan, W.H., Huang, J.J., Huang, W.X., Wang, Y. and Zheng, W.F. (2017). Production of a fungal furocoumarin by a polyketide synthase gene cluster confers the chemo-resistance of Neurospora crassa to the predation by fungivorous arthropods. Environmental Microbiology 19: 3920-3929. 


\title{
Chapter 2: Effect of animal predation on the biomass of mycotoxin- producing fungi
}

\author{
Yang Xu, Petr Karlovsky* \\ Molecular Phytopathology and Mycotoxin Research, Georg-August-University Göttingen \\ Grisebachstr. 6, 37077 Göttingen, Germany \\ *Corresponding author
}

\begin{abstract}
Interactions between fungi and invertebrates nearly occur in every ecosystem. Grazing affects fungal biomass density and is species-dependent. However, how invertebrates affect mycotoxin-producing fungi has not been studied yet. In our study, we exposed three well-known mycotoxin-producing Fusarium strains (F. graminearum, F. culmorum and $F$. verticillioides), two Aspergillus strains (A. nidulans and A. ochraceus) and one Penicillium strain ( $P$. viridicatum) to intense grazing by Folsomia candida and determined fungal biomass gravimetrically and by quantitative real-time PCR. Our results indicated that only $A$. nidulans and $F$. verticillioides were consumed in larger amounts by the collembolan. The mycelial biomass of $A$. ochraceus even increased after grazing commenced. Our results suggest that collembolan grazing caused variable effects on the mycelial growth of mycotoxin-producing fungal strains but no uniform reduction.
\end{abstract}

Keywords: Fusarium, Aspergillus, Penicillium, Folsomia candida, mycelial growth quantitation 


\section{Introduction}

As decomposition clears in the field, fungi provide temporary habitat and rich nutrition to the animals (Fogel \& Trappe, 1978; Scheu et al., 2004a, b). Gut content analysis revealed that collembolans consume microfungi, among other food resources (Kaneko et al., 1998; Ponge 2000). Invertebrates are abundant in natural and agricultural soils and some dependent on fungi as food source (Innocenti et al., 2009). Interaction between fungi and invertebrate is common, and the effect of small animal feeding on fungi is ambiguous. Whether it is cord persistence, compensatory or mycelium removal depends on fungal species, fungal structure, age, physiological status, nutrient content and biochemistry (Crowther et al., 2011; 2012). Ciid beetles (Octotemmus glabriculus and Cis bolete) reduce reproductive potential of their host fungus by $50 \%$ both by inhibiting mycelium growth and reproductive fitness (Guevara et al., 1999). Conversely, Hasan (1993) found that in the presence of the nematode Meloidogyne, the severity of Fusarium wilt in cotton root increased.

Previous studies suggest that, generally, low grazing can stimulate fungal development whereas intensive grazing affects the fungal biomass negatively (Crowther et al., 2012). Moreover, different invertebrate species can affect the same fungal species in different ways, and conversely, different fungi tolerate grazing by the same invertebrate species variably (Scheu \& Simmerling, 2004; Tordoff et al., 2007). The interactions have shown to even affect wood and litter decay rates in soil ecosystems (Crowther et al., 2011).

Mycotoxin-producing fungi (Fusarium spp., Aspergillus spp. and Penicillium spp.) cause diseases in plants, animals and human beings. Aspergillus spp., Penicillium spp. are known as unattractive food sources to fungal-grazing invertebrates (Scheu et al., 2004; Maraun et al., 2003). Fusarium spp. are reported to be attractive for collembolans that graze on fungi (Jorgensen et al., 2003). Cases that mycotoxin-producing fungi attract invertebrates have been reported (Schulthess et al., 2002; Guo et al., 2014). However, the effect of invertebrate grazing on fungal biomass development has been neglected so far. This study compares the mycelial growth of several mycotoxin-producing fungal strains, including $F$. graminearum, F. culmorum, $F$. verticillioides, A. nidulans, A. ochraceus and $P$. viridicatum, on mycelium development in presence and absence of $F$. candida grazing. Mycelium growth was assessed both by weight determination and real time PCR.

\section{Material and methods}




\subsection{Fungal strains and animals}

F. graminearum IFA66 was obtained from Marc Lemmens (IFA Tulln, Austria) via Thomas Miedaner (University of Hohenheim, Germany) (Miedaner et al., 2010). and $F$. culmorum 3.37 were acquired from Prof. Heinz-Wilhelm Dehne, University of Bonn (Guo et al. 2014). A. ochraceus and P. viridicatum were purchased from DSMZ (German Collection of Microorganisms and Cell Cultures, Braunschweig, Germany). The strain $F$. verticillioides Ita 1 was kindly donated by Francesca Cardinale (Turin University, Italy). The strain A. nidulans RDIT 2.3 and Folsomia candida were obtained from Prof. Dr. Marko Rohlfs (University of Göttingen, Germany). Collembola was cultured in $9 \mathrm{~cm}$ petri dishes with a substrate mixture of plaster of Paris:Charcoal (9:1). They were incubated in darkness at $15^{\circ} \mathrm{C}$ and fed on baker's yeast.

\subsection{Fungal exposure to collembolans}

Media were prepared by adding six rice kernels to $1 \mathrm{~mL}$ demineralized water were added to Erlenmeyer flasks and autoclaved. $F$. graminearum and $F$. verticillioides fungal plugs served as inoculum respectively. They were cultured at $15^{\circ} \mathrm{C}$ in the dark for 10 days. Adding proper amount of autoclaved water helped to maintain humidity. Then, $60 \mathrm{mg}$ of two-day starved $F$. candida animals were put into the flasks to consume the fungi, whilst no collembolans were added to the control. Three days later, at least four replicates of control and treatment samples were harvested.

Another medium was prepared by adding 1-3 rice kernels and 90-200 $\mu \mathrm{L}$ demineralized water into tubes, which were autoclaved. Then spore suspensions $(1000 \mathrm{spore} / \mu \mathrm{l})$ of $F$. graminearum, F. culmorum, A. ochraceus, A. nidulans and $P$. viridicatum were used as inoculum. The tube cultures were incubated at $15-18^{\circ} \mathrm{C}$ for $5-7$ days in the dark. After that, approximately $20 \mathrm{mg}$ of two-day starved $F$. candida animals were put into the falcon tube to graze for various days, respectively. Controls were kept animal-free. Loosened falcon lids provided minimal air supply. At least 3 replicates were harvested at each time points for each group.

2.3. Preparation of DNA standards for the determination of A. ochraceus, A. nidulans and $\boldsymbol{P}$. viridicatum biomass 
Fungal DNA was extracted from A. ochraceus, A. nidulans and P. viridicatum mycelium, all of which were cultured on PDA medium, by using CTAB method (Brandfass \& Karlovsky. 2008). DNA concentrations were determined by using an Epoch microplate spectrophotometer (BioTek, Vermont, US). After that, DNA extracts were diluted five times and run on fresh $0.8 \%$ agarose gels with Lambda DNA with known concentration in dilution series together. Photos were taken under in a digital imaging system (Viber Lourmat, Eberhardzell, Germany) Fungal DNA concentrations were determined by using image $J$ with $\lambda$ DNA as reference. These DNA were kept as stock solution for quantitative PCR.

Before using as a quantification standard, the fungal DNA extracts were diluted with $0.5 \times \mathrm{TE}$ buffer to $100 \mathrm{pg} / \mu \mathrm{L}$ as stock solution and stored at $-20^{\circ} \mathrm{C}$. Whenever quantification was needed, a three-fold series dilution of the stock solution was prepared, $100,33,11,3.7,1.2,0.4$ and $0.1 \mathrm{pg} / \mu \mathrm{L}$.

\subsection{DNA extraction of grazed and non-grazed fungal mycelia}

Each fungal species sample from the springtail bioassays (treatments and controls) were harvested and frozen at $-20^{\circ} \mathrm{C}$ for two days. Then the samples were freeze-dried for three days.Then fungal mycelial were ground in a mill MM 200 (Retsch, Haan, Germany) for 1$2 \mathrm{~min}$. Genomic DNA was extracted from 10-20 mg mycelial powder by using CTAB extraction method (Brandfass \& Karlovsky. 2008). DNA quality was checked in $0.8 \%$ agarose gel as described previously.

\subsection{Quantification of fungal DNA by qPCR}

Each fungal genomic DNA was subsequently diluted in the range of standard curve (0.1$100 \mathrm{pg} / \mu \mathrm{L}$ ). Real time PCR reaction were performed in 384-well plates (Kisker Biotech $\mathrm{GmbH}$, Steinfurt, Germany) with $4 \mu \mathrm{L}$ reaction system in a CFX384 thermocycler (BioRad, California, US).

F. verticillioides, $F$. culmorum, $F$. graminearum real time PCR were performed under the similar conditions as described by Dastjerdi (2008) with slightly modified conditions according to reagents manufacturer's request. A. nidulans, A. ochraceus and $P$. viridicatum PCR conditions were optimized with $\mathrm{MgCl}_{2}$ and annealing temperature was determined before any sample analysis. Real time PCR was performed with $4 \mu \mathrm{L}$ reaction system consisting of $1 \times$ buffer (New England Bioline, London, UK), 2-4.5 mM MgCl 2 (Nippon Genetics Europe GmbH, Dueren, Germany), $150 \mu \mathrm{M}$ dNTP (Bioline, Luckenwalde, 
Germany), $0.3 \mu \mathrm{M}$ respective primers, $0.1 \times$ SYBR green I (Molecular Probes, Eugene, Oregon, US), 0.025U/ $\mu \mathrm{L}$ Taq polymerase (New England Bioline, London, UK). PCR was performed with denaturation for $2 \mathrm{~min}$ at $94^{\circ} \mathrm{C}$, followed by 39 cycles of denaturation at $94^{\circ} \mathrm{C}$ for $30 \mathrm{~s}$, annealing at $58-64^{\circ} \mathrm{C}$ for $30 \mathrm{~s}$, and primer extension at $68^{\circ} \mathrm{C}$ for $30 \mathrm{~s}$, with fluorescence acquisition step at the end of the extension. Melting analysis of the PCR product was initiated by an incubation of $68^{\circ} \mathrm{C}$ for $5 \mathrm{~min}, 95^{\circ} \mathrm{C}$ for $1 \mathrm{~min}$ and cooling to $55^{\circ} \mathrm{C}$ for $1 \mathrm{~min}$ before melting curve acquisition by raising the temperature to $95^{\circ} \mathrm{C}$ at a rate of $0.5^{\circ} \mathrm{C} / \mathrm{s}$ with continuous fluorescence acquisition. A final cooling step was performed at $20^{\circ} \mathrm{C}$ for $10 \mathrm{~min}$. Table summarizes the used primers. The calibration curve $\mathrm{R}^{2}>98 \%$ was taken as quality standard.

Table 1: Primers used to quantify various fungal species

\begin{tabular}{|c|c|c|c|c|}
\hline Target species & Primer name & 5 '-3' sequence & $\begin{array}{l}\text { Fragment } \\
\text { size }\end{array}$ & $\begin{array}{c}\text { Reference/ } \\
\text { Accession No. }\end{array}$ \\
\hline F. culmorum & $\begin{array}{l}\text { Fg OPT 18F } \\
\text { Fg OPT 18R }\end{array}$ & $\begin{array}{l}\text { GATGCCAGACCAAGACGAAG } \\
\text { GATGCCAGACGCACTAAGAT }\end{array}$ & 472 bp & $\begin{array}{c}\text { Schilling et al., } \\
\text { (1996) }\end{array}$ \\
\hline F. verticillioides & $\begin{array}{l}\text { VER } 1 \\
\text { VER } 2\end{array}$ & $\begin{array}{c}\text { CTTCCTGCGATGTTTCTCC } \\
\text { AATTGGCCATTGGTATTATATATCTA }\end{array}$ & $578 b p$ & $\begin{array}{l}\text { Mulè et al., } \\
2004\end{array}$ \\
\hline F. graminearum & $\begin{array}{l}\mathrm{Fg} 16 \mathrm{NF} \\
\mathrm{Fg} 16 \mathrm{NR}\end{array}$ & $\begin{array}{l}\text { CTCCGGATATGTTGCGTCAA } \\
\text { GGTAGGTATCCGACATGGCAA }\end{array}$ & $280 \mathrm{bp}$ & $\begin{array}{l}\text { Nichloson et al. } \\
1998\end{array}$ \\
\hline A. nidulans & $\begin{array}{l}\text { AflR F } \\
\text { AflR R }\end{array}$ & $\begin{array}{l}\text { AGAGCCGCATGAGAGTATCC } \\
\text { CTCGCTTTCTCCTTCGCTTC }\end{array}$ & $206 \mathrm{bp}$ & U34740.1 \\
\hline A. ochraceus & $\begin{array}{l}\text { Ao Tub F } \\
\text { Ao Tub R }\end{array}$ & $\begin{array}{l}\text { CGCGTTTACACCCATCGAAA } \\
\text { CCACCGGAAGCCTAGAAGAT }\end{array}$ & $245 \mathrm{bp}$ & KX421550.1 \\
\hline P. viridicatum & $\begin{array}{l}\text { Pv Tub F } \\
\text { Pv Tub R }\end{array}$ & $\begin{array}{l}\text { GGGGTTTCCGGTAGATCACA } \\
\text { GGCTCCAAATCGACGAGAAC }\end{array}$ & $212 b p$ & AY674294.1 \\
\hline
\end{tabular}

\subsection{Statistical}

Bar graphs show means \pm SE. Significance of difference between means was tested by unpaired t-test by using Gaphpad Prism 5. The number of biological replicates is always more than three.

\section{Results}

\subsection{Standard curve of each fungal strain for real-time PCR}


After comparing $\mathrm{Ct}$ values of real time PCR test runs, optimized $\mathrm{MgCl}_{2}$ concentration and annealing temperature were obtained as follows: A. ochraceus $-4.5 \mathrm{mM}$ and $63^{\circ} \mathrm{C} ; P$. viridicatum $-2 \mathrm{mM}$ and $63^{\circ} \mathrm{C} ; \mathrm{A}$. nidulans $-2 \mathrm{Mm}$ and $59.1^{\circ} \mathrm{C}$. In addition, by using these $\mathrm{MgCl}_{2}$ concentrations and annealing temperature, the efficiency and $\mathrm{R}^{2}$ for all linear calibration curves were more than $98 \%$ (Figure 1). Therefore, we used these linear calibration curves to perform further analysis.
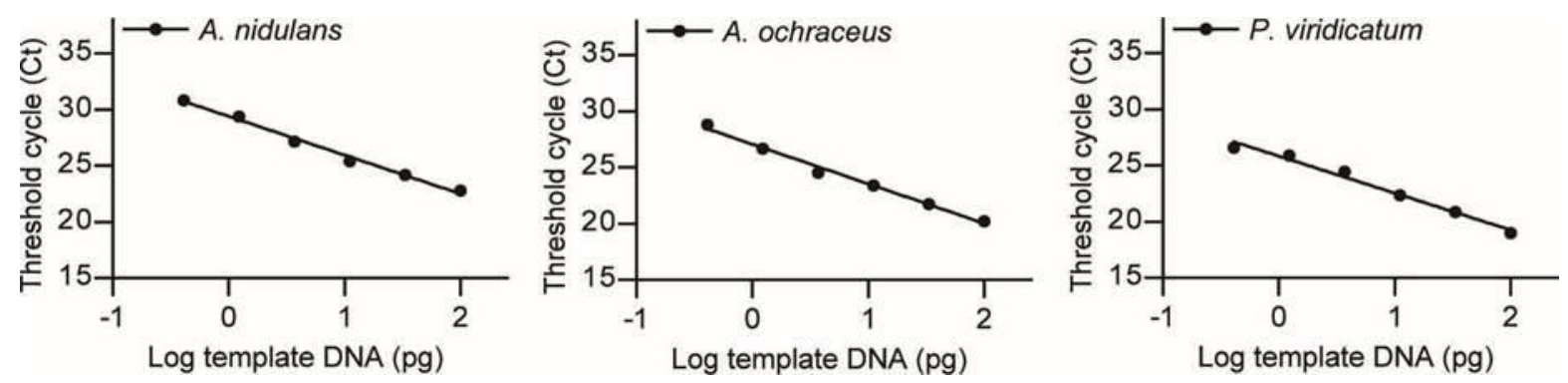

Figure 1: Standard curve for A. nidulans $\left(\mathrm{R}^{2}=99.16 \%\right)$, A. ochraceus $\left(\mathrm{R}^{2}=99.05 \%\right)$ and $P$. viridicatum $\left(\mathrm{R}^{2}=98.42 \%\right)$.

\subsection{Fungal weight after animal predation}

In general, grazing by $F$. candida caused the dry weight of the mycelium no to differ from that of the intact control group, and A. nidulans was the only exception. The dry weight of grazed A. nidulans mycelium was gradually reduced in 43 days, at most time points of analysis even present significant difference (Figure 2).

\subsection{DNA quality}

Large amounts of genomic DNA were visible on the agarose gel lacking any signs of degradation. However, the DNA quality for A. nidulans was quite poor (see supplement Figure 1). Therefore, the real time PCR analysis was continued by using genomic DNA extracted from all the fungal species except for A. nidulans. 

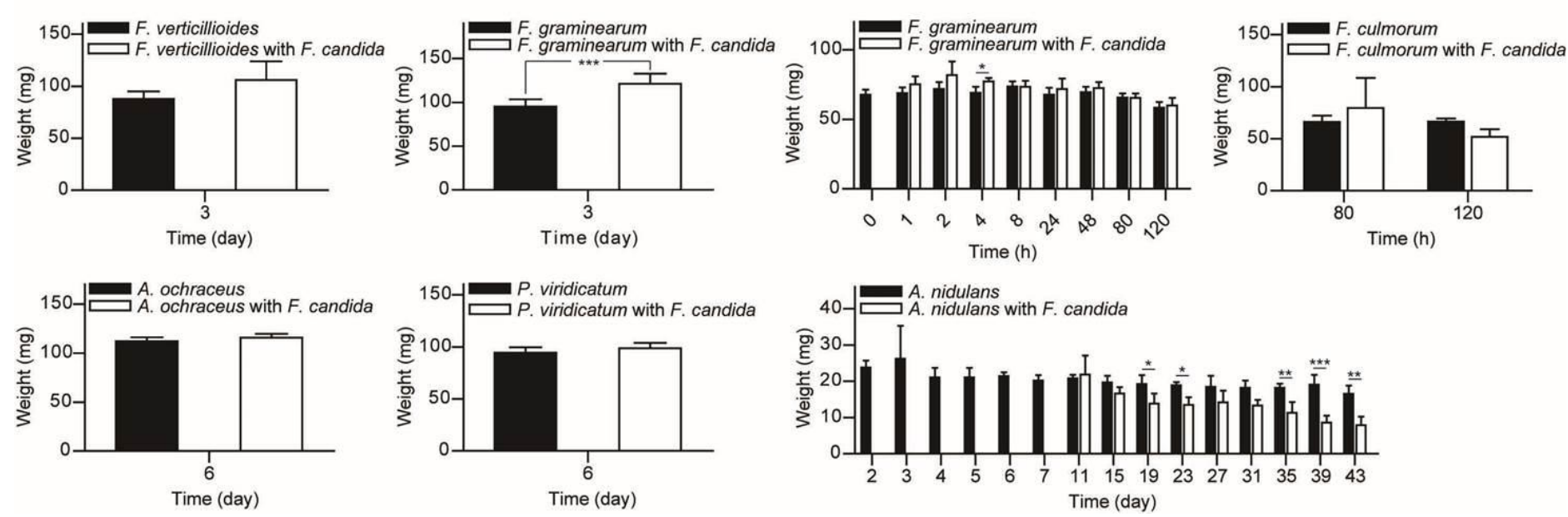

Figure 2: Mycelium weight of each fungal species when grazed by springtails and unattacked $(* 0.05<\mathrm{P}<0.01, * * 0.01<\mathrm{P}<0.001$, *** $\mathrm{P}<0.001)$ 


\subsection{Fungal biomass assessment by real-time PCR}

Due to the poor-quality genomic DNA from A. nidulans, real-time PCR test for this fungal species could not be performed. The other fungal strains (Figure 3) did not show differ in terms of DNA amount when unharmed and grated samples were compared. A. ochraceus mycelium growth appeared even to be stimulated by feeding. F. verticillioides was the only tested fungal strain that was negatively affected by feeding by producing lower amounts of mycelium in the presence of $F$. candida. 

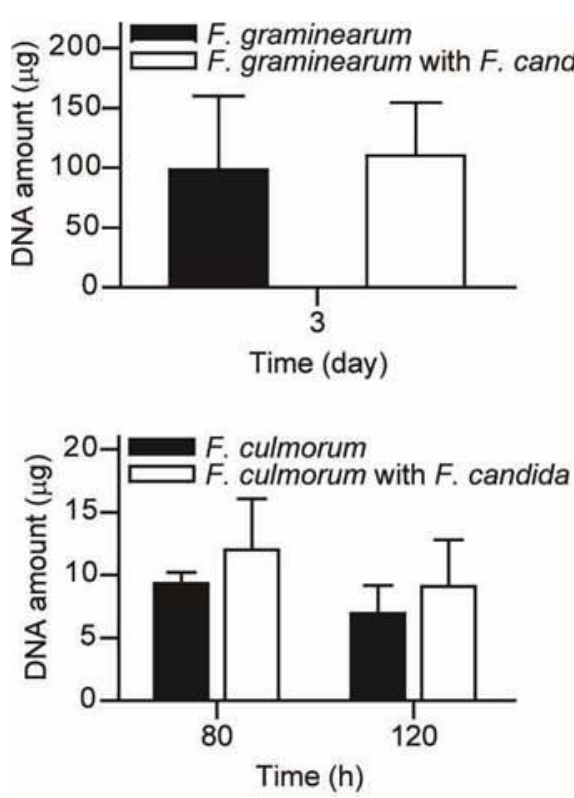
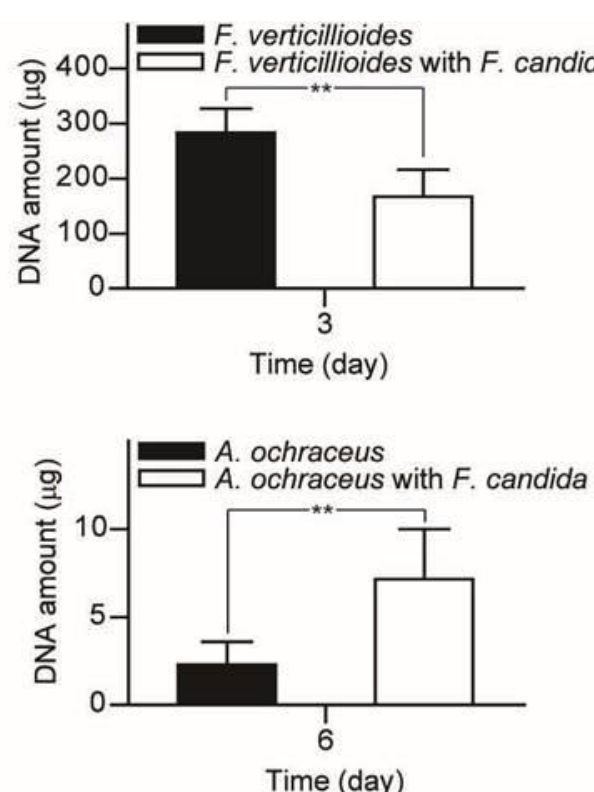
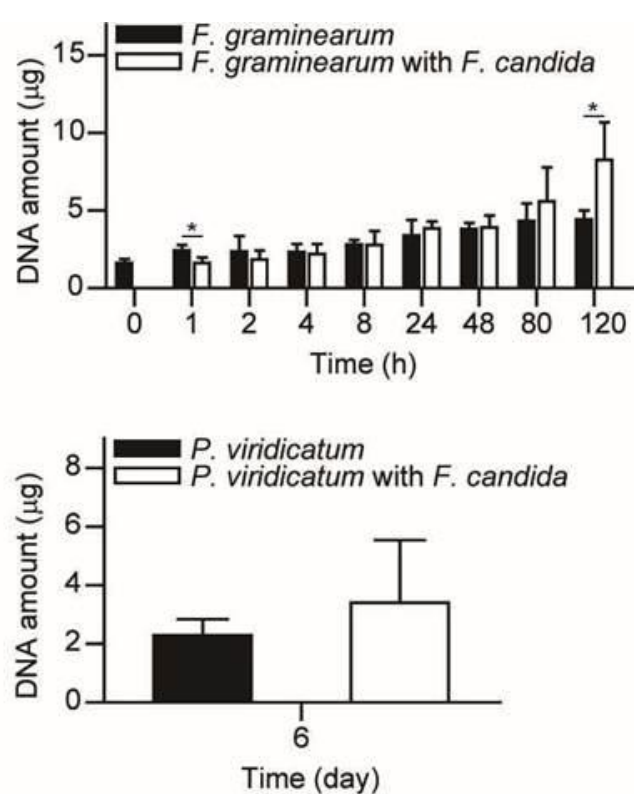

Figure 3: DNA amount of each fungal species quantified by quantitative real time PCR when treated with and without springtails $(* 0.05<\mathrm{P}<0.01, * * 0.01$ $<\mathrm{P}<0.001)$ 


\section{Discussion}

The primers used to detect the amount of A. ochraceus and $P$. viridicatum DNA were good enough to quantify the respective fungal species only. However, it should not be used in multi-fungal species samples since their specificity range has to be determined yet. Moreover, tubulin is an essential gene for all fungal species. It is possible it could amplify other unspecific Aspergillus spp. as well. On the other hand, primers for Fusarium spp.are chose from previous research, they approved to harbor well specificity.

After consumption by springtails, the dry weight of $F$. graminearum, $F$. culmorum, $F$. verticillioides, $P$. viridicatum and A. ochraceus indicated no inhibition by grazing. This might be caused by the fact that no animals were removed before weighing, thereby contributing to some portion of the weight of the treated groups. However, in case of $A$. nidulans, even without deducting the weight of $F$. candida, the dry weight of this fungal species decreased gradually.

DNA quality of $A$. nidulans was poor, which might be due to the cell wall chemistry and induced production of spores on rice medium. By contrast, for all other fungal species, the DNA extraction and real time PCR quantification worked well.

The amount of genomic DNA of $F$. graminearum, $F$. culmorum, $P$. viridicatum and $A$. ochraceus showed no reduction of mycelial weight when grazed and non-gazed treatments were compared Aspergillus and Penicillium are well known as an untractive diet source for the animals. Various secondary metabolites, peptides, proteins production might contribute to collembolans avoiding these fungi (Stötefeld et al., 2012).

Cases are known that low density grazing of collembolan can stimulate fungal growth. Conversely, under heavy grazing pressure, fungal growth can stop (Crowther et al., 2012). However, in our case, when large amount of springtails were added to fungal mycelium grown on three-rice-kernel, the fungus probably under higher stress than in the natural environment. Still, the fungal biomass was not lower when grazed.

Real time PCR indicated reduced growth of $F$. verticillioides when grazed. Which is inconsistent with weight determination results, and might due to rice substance take account large proportion of weight while fungal only make up a small percentage of that. Schulthess et al., (2002) reported that $F$. verticillioides can act as a growth promotor, for example as observed for Eldana saccharina and Cryptophlebia leucotreta, two insects that 
are attracted to $F$. verticillioides-infected maize-stems. Their survival rate and growth improved compared to uninfected stems. Bores and beetle numbers also were higher in the F. verticillioides inoculated rows than in A. flavus inoculated rows in another experiment (Cardwell et al., 2000). The potentially stimulating effects of $F$. verticillioides on grazers could explain the detected developmental impairment by grazing. Growth stimulation of insects has been reported for other fungi too (Pelizza et al., 2010).

Wolfarth et al. (2013) reported that the nematode A. saprophilus and the collembolan $F$. candida reduce the biomass of $F$. culmorum, which is the other way around with our study. The contrasting results might be explained the use of different analysis methods and different experimental condition. For example, Wolfarth et al. (2013) were using ELISA assays to quantify Fusarium protein equivalents, which might not detect spore amounts as accurately as real time PCR (Perestam et al., 2016).

In our study, total DNA amount of fungi were revealed by real time PCR. This method is not capable of distinguishing the fungal mycelium from spores. It is known that harsh growth conditions (perhaps including consumption by animals) stimulate the production of fungal spores (Su et al., 2002). The effect of spore production of mycelial growth warrants more detailed explorations.

Pathogen outbreaks in the field can be accelerated by insect as propagation vectors (Hasan, 1993), or decreased by animal consuming pathogenic fungi (Guevara et al., 1999). In this laboratory study, the effects of collembolan grazing on selected fungal strains yielded results that concurred with the line of expectations mycotoxin-producing fungi survive collembolan-grazing attacks. However, some stimulating and inhibitory effects were observed in single cases. Real time PCR proved as more sensitive than mycelial weight determination. Grazing pressure could represent an important that affects the outcome of such studies. For example, woodlice are known to exert a more extreme grazing pressure to fungal communities than collembolans (Crowther et al., 2011; 2012). Moreover, qualitative and quantitative effects of mycotoxin production have to be taken into account for a more insightful understanding of effects that fungal grazers can cause on fungal communities (Fandohan et al. 2006). 


\section{References}

Brandfass, C. and Karlovsky, P. (2008). Upscaled CTAB-based DNA extraction and realtime PCR assays for Fusarium culmorum and F.graminearum DNA in plant material with reduced sampling error. International Journal of Molecular Sciences 9: 2306-2321.

Cardwell, K.F., Kling, J.G., Maziya-Dixon, B. and Bosque-Perez, N.A. (2000). Interactions between Fusarium verticillioides, Aspergillus flavus, and insect infestation in four maize genotypes in lowland Africa. Phytopathology 90: 276284.

Crowther, T.W., Jones, T.H., Boddy, L. and Baldrian, P. (2011). Invertebrate grazing determines enzyme production by basidiomycete fungi. Soil Biology \& Biochemistry 43: 2060-2068.

Crowther, T.W. and A'bear, A.D. (2012). Impacts of grazing soil fauna on decomposer fungi are species-specific and density-dependent. Fungal Ecology 5: 277-281.

Dastjerdi, R. (2014). High fumonisin content in maize: search for source of infection and biological function. Ph. D. Thesis, University of Goettingen, Germany.

Fandohan, F., Gnonlonfin, B., Hell, K., Marasas, W.F.O. and Wingfield, M.J. (2006). Impact of indigenous storage systems and insect infestation on the contamination of maize with fumonisins. African Journal of Biotechnology 5: 546-552.

Fogel, R. and Trappe, J.M. (1978). Fungus consumption (Mycophagy) by small animals. Northwest Science. 52: 1-31.

Guevara, R., Rayner, A.M. and Reynolds, S. (1999). Effects of fungivory by two specialist ciid beetles (Octotemnus glabriculus and Cis boleti) on the reproductive fitness of their host fungus, Coriolus versicolor. New Phytologist 145: 137-144.

Guo, Z.Q., Döll, K., Dastjerdi, R., Karlovsky, P., Dehne, H.W. and Altincicek, B. (2014). Effect of fungal colonization of wheat grains with Fusarium spp. on food choice, weight gain and mortality of meal beetle larvae (Tenebrio molitor). Plos One. 9: e100112. https://doi.org/10.1371/journal.pone.0100112

Hasan, A. (1993). "The role of fungi in fungus-nematode interactions." In: Nematode Interactions (M. W. Khan, ed.) Springer, Dordrecht. pp 273-287. 
Innoceti, G., Sabatini, M.A., Ganassi, S., Montanari, M. and Branzanti, M.B. (2009). Trophic interactions between soil fungi and collembola. Multitrophic interactions in soil. IOBC Bulletin 42: 91-94.

Jorgensen, H.B., Elmholt, S. and Petrsen, H. (2003). Collembola dietary specialisation on soil grown fungi. Biology and Fertility of Soils 39: 9-15.

Maraun, M., Martens, H., Migge, S., Theenhaus, A. and Scheu, S. (2003). Adding to 'the enigma of soil animal diversity': fungal feeders and saprophagous soil invertebrates prefer similar food substrates. European Journal of Soil Biology. 39: 85-95.

Miedaner, T., Bolduan, C. and Melchinger, A.E. (2010). Aggressiveness and mycotoxin production of eight isolates each of Fusarium graminearum and Fusarium vertillioides for ear rot on susceptible and resistant early maize. European Journal of Plant Pathology 127: 113-123.

Mule, G, Susca, A, Stea, G, Moretti, A. (2004). A species-specific PCR assay based on the calmodulin partial gene for the identification of Fusarium verticillioides, $F$. proliferatum and F. subglutinans. European Journal of Plant Pathology 110: 495502.

Nicholson, P., Simpson, D.R., Weston, G., Rezanoor, H.N., Lees, A.K., Parry, D.W. and Joyce, D. (1998). Detection and quantification of Fusarium culmorum and Fusarium graminearumin cereals using PCR assays. Physiological and Molecular Plant Pathology 53: 17-37.

Pelizza, S.A., Stenglein, S.A., Cabello, M.N., Dinolfo, M.I. and Lange, C.E. (2010). First record of Fusarium verticillioides as an entomopathogenic fungus of grasshoppers. Journal of Insect Science 11: 1-8.

Ponge, J.F. (2000). Vertical distribution of Collembola (Hexapoda) and their food resources in organic horizons of beech forests. Biology and Fertility of Soils 32: $508-522$.

Perestam, A.T., Fujisaki, K.K., Nava, O., Hellberg, R.S. (2017). Comparison of real-time PCR and ELISA-based methods for the detection of beef and pork in processed meat products. Food Control 71: 346-352

Scheu, S. and Folger, M. (2004). Single and mixed diets in collembolan: effects on reproduction and stable isotope fractionation. Functional Ecology 18: 94-102 
Schulthess, F., Cardwell, F. and Gounou, S. (2002). The effect of endophytic Fusarium vertcillioides on infestation of two maize varieties by lepidopterous stemborers and coleopteran grain feeders. Phytopathology 92: 120-128.

Stötefeld, L., Scheu, S. and Rohlfs, M. (2012). Fungal chemical defence alters densitydependent foraging behavior and success in a fungivorous soil arthropod. Ecological Entomology 37: 323-329.

Su, Y.Y., Qi, Y.L. and Cai, L. (2012). Induction of sporulation in plant pathogenic fungi. Mycology 3 (3): 195-200.

Tordoff, G.M., Boddy, L. and Jones T.H. (2007). Species-specific impacts of collembola grazing on fungal foraging ecology. Soil Biology \& Biochemistry 40: 434-442.

Wolfarth, F., Schrader, S., Oldenburg, E. and Weinert, J. (2013). Nematode-collembolaninteraction promotes the degradation of Fusarium biomass and deoxynivalenol according to soil texture. Soil Biology \& Biochemistry 57: 903-910. 


\section{Extended data}

A. nidulans/ $A$. nidulans with $F$. candida $\lambda \mathrm{DNA}$

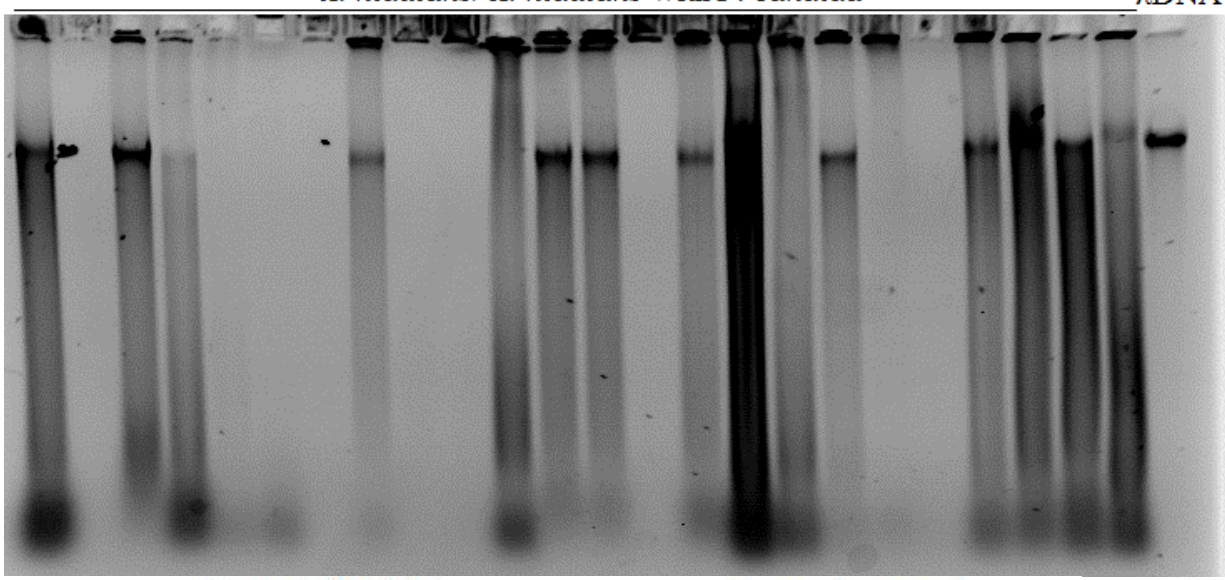

F. verticillioides/

F. graminearum/

$\lambda$ DNA $F$. verticillioides with $F$. candida $\quad F$. graminearum with $F$. candida

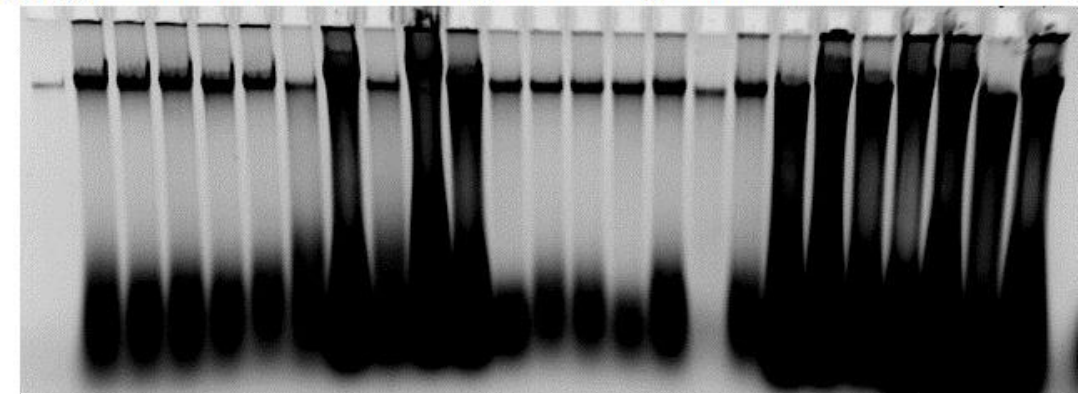

$\lambda \mathrm{DNA}$

F. culmorum/ F. culmorum with $F$. candida

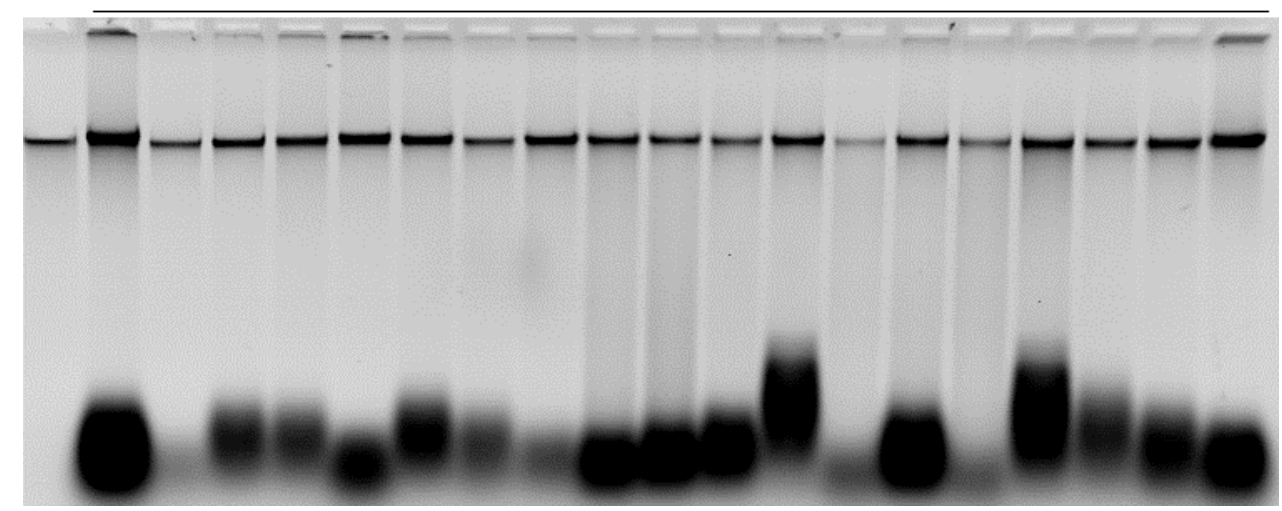

Extended Data Fig. 1 | Gel electrophoresis of DNA extraction yield from some fungal species when treated with and without springtails. $\lambda \mathrm{DNA}$ as marker. 


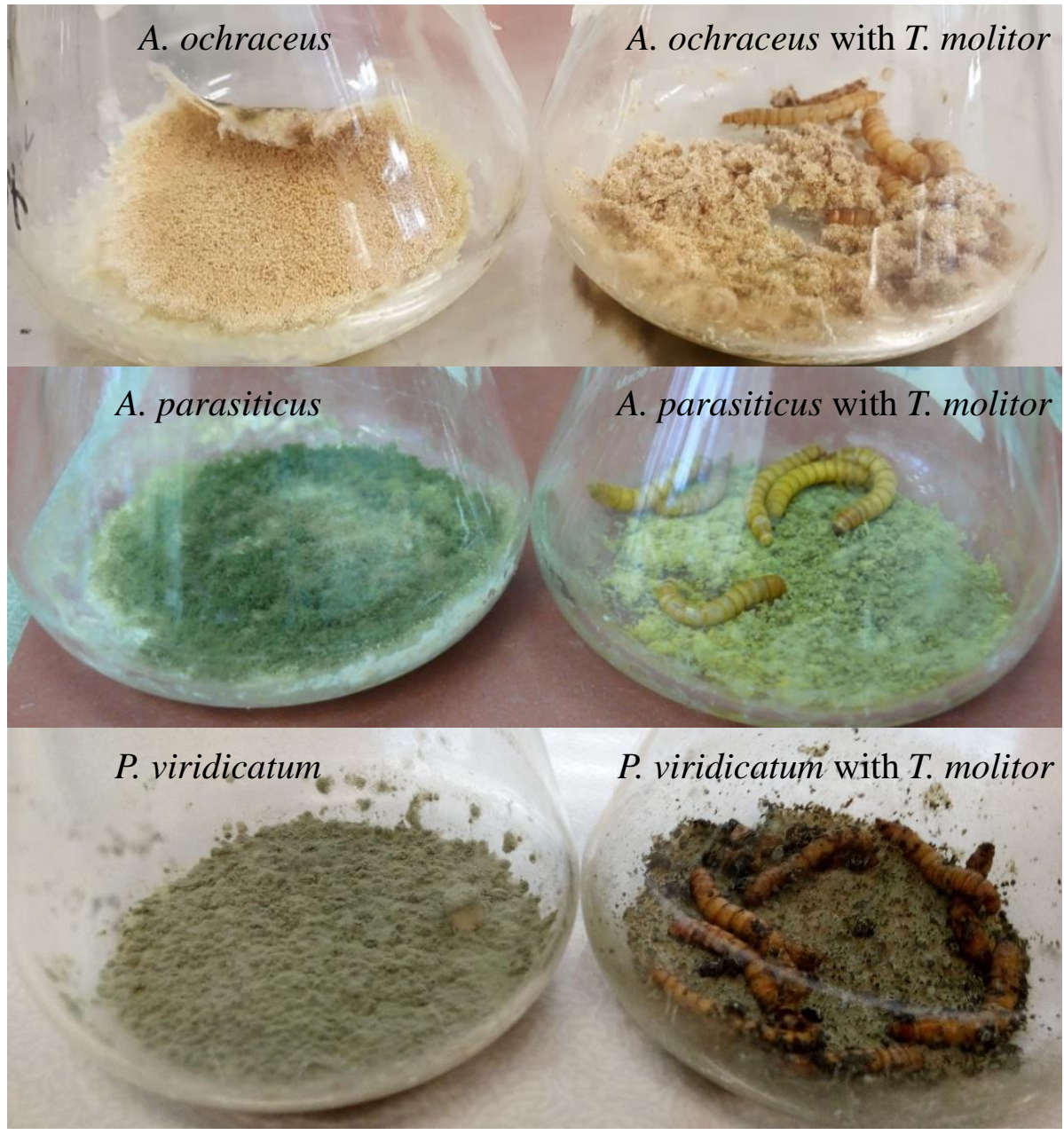

Extended Data Fig. 2 | Photos of various mycotoxin-producing fungal species 5 days after treating with and without mealworms (T. molitor). And invertebrate consumption causes no negative effect on fungal (Aspergillus spp. and Penicillium spp.) biomass. 


\title{
Chapter 3: Investigation of interactions between Fusarium graminearum, Fusarium verticillioides and Folsomia candida (Collembola)
}

\author{
Yang Xu, Petr Karlovsky* \\ Molecular Phytopathoology and Mycotoxin Research, Georg-August-University Göttingen \\ Grisebachstr. 6, 37077 Göttingen, Germany \\ *Corresponding author
}

\begin{abstract}
Collembolans are selective feeders. Their food preference contributes to shaping of fungal community composition in various ecosystems. Previous experiments showed that $F$. candida grazing caused different fungal biomass loss effect on F. verticillioides and $F$. graminearum. This is intriguing regarding to their biological similarity. In this study, we explored the food preference of the springtail Folsomia candida between F. verticillioides and $F$. graminearum in terms of various aspects: (1) which spies is more attractive, $F$. verticillioides or $F$. graminearum; (2) how attractive are the mycotoxin-deficient $F$. graminearum strains, $\triangle \mathrm{DON}$ (lacks deoxynivalenol), $\triangle \mathrm{ZEN}$ (lacks zearalenone), $\triangle \mathrm{AUR}$ (lacks aureofusarin); and (3) to which extent does mixing of cultures affect collembolan grazing. Collembolan activity was determined by eye observation and real time PCR of DNA mycelium extracts. In our assays, $F$. candida prefers $F$. verticillioides and avoids $F$. graminearum. However, the $\triangle$ AUR $F$. graminearum is attractive to $F$. candida over $F$. verticillioides. In addition, springtails prefer mixtures of two fungal strains to a single strain. Even the avoided F. graminearum is consumed in higher amounts, when another fungal strain is present. The obtained provide support for the hypothesis that fungal secondary metabolitecan contribute to the avoidance of a specific fungal species by a collembolan grazer. Additionally, a fungal strain that produces a feeding deterrent compound can be consumed in higher amounts in a mixed community than a sole food source.
\end{abstract}

Keywords: F. verticillioides, $F$. graminearum, food preference, aurofusairn, single culture, mixed culture 


\section{Introduction}

Springtails (Collembola) represent a major class of soil animals which contribute to the fragmentation of soil organic matter and are thought to exert control effects on soil microbial communities (Hopkin, 1997). Many studies have focused on their food preferences (Jorgensen et al., 2003; Kaneda \& Kaneko, 2004; Varga et al., 2002). Gut content analyses revealed that collembola consume considerable portions of fungal hyphae (Ponge, 2000). Numerous studies have reported that collembolans prefer saprophytic to mycorrhizal fungi (Bardgett et al. 1993; Schneider \& Maraun, 2005; Jonas et al., 2007).

Fungi produce diverse secondary metabolites (Turner \& Aldridge 1983). Many of these fungal secondary metabolites possess toxic properties and as mycotoxins underlie strict control regulations concerning their concentrations in food stuffs. According to general assumptions, collembola deal with this challenge by mixing their diet to be comprised of different fungal species. A combination of toxins and high-quality food seems to represent a more balanced as well as tolerable food supply (Haggle and Rowell-Rahier 1999; Bernay and Minkenberg 1997; Bernays et al., 1994; Rapport 1980; Pullman 1975; Freeland and Janzen 1974).

Studies have shown that the quality of volatile and non-volatile secondary metabolites, mycelial age and its metabolic activity determine the food preference of collembola (Bengtsson et al., 1988; Rohlfs et al., 2007; Döll et al., 2013). Preference of F. candida for certain fungal species is well established: several fungal species colonies were placed at a distance in an arena and the number of collembolans visiting the colonies monitored. However, in natural environment, colonization of organic substrates by a mixture of different fungal species is common. Therefore, determining food preference of collembola with mixed culture instead of single colony is essential to study collembolan behavior in natural environments (Kaneda \& Kaneko, 2004).

As plant pathogens, Fusarium species cause seedling blight and root, stalk and ear rot worldwide. In this context, it is interesting to note that collembolans prefer Fusarium species as diet (Innocenti et al., 2009). Deoxynivalenol (DON), zearalenone (ZEA) and fumonisin B1 (FB1) belong to the most common secondary metabolites that are produced by $F$. graminearum and $F$. verticillioides, and, consequently, are the most common mycotoxins found in corn (Marin et al. 2006). And they uaually present color, because production of aurofusarin or bikaverin. 
We hypothesize that the differential secondary metabolite production of $F$. graminearum and $F$. verticillioides influences the food preference of $F$. candida between the two species.

In addition, the food source of collembola in a natural ecosystem is very complex. Therefore, we hypothesized that collembola may not recognize their preferred food in a mixed assay as well as when the fungi are served separately. In this study, traditional food preference assay and molecular techniques (real-time PCR) were used to investigate the food selection behavior of $F$. candida between $F$. graminearum and $F$. verticillioides.

\section{Material and Method}

\subsection{Test organisms}

Folsomia candida was kindly donated by Prof. Dr. Marko Rohlfs (Georg-August University of Göttingen, Germany) and was cultured in $9 \mathrm{~cm}$ petri dishes with a substrate mixture of plaster of Paris:Charcoal (9:1). The collembola were incubated in darkness at $15^{\circ} \mathrm{C}$ and fed on baker's yeast.

Fusarium verticillioides Ita 1 was kindly donated by Francesca Cardinale (Turin University, Italy), and F. graminearum strain IFA 66 (DON-chemotype) was received from M. Lemmens, University of Hohenheim, Stuttgart, Germany (Miedaner et al., 2010). F. graminearum $\triangle \mathrm{DON}, F$. graminearum $\triangle \mathrm{ZEA}, F$. graminearum $\Delta \mathrm{AUR}$ (Malz et al., 2005), were kindly provided by W. Schäfer, University of Hamburg, Germany.

\subsection{Food preference of $F$. candida}

\subsubsection{Choice assay between Fusarium graminearum and $F$. verticillioides}

Spores $\left(10^{3}\right.$ spores/ $\left.\mu \mathrm{L}\right)$ of $F$. verticillioides Ita 1 and $F$. graminearum IFA 66 were cultured onto $20 \mathrm{~mL}$ homemade potato dextrose broth medium in a $100 \mathrm{~mL}$ flask at $25^{\circ} \mathrm{C}$ for 7 days in the dark. Mycelia were harvested from the edge using a sterile cork borer (12 $\mathrm{mm}$ diameter). Mycelial samples of both strains were placed on opposite sides of $9 \mathrm{~cm}$ petri dishes previously filled with a layer of plaster of Paris:activated charcoal (9:1). 20 two-day hundried $F$. candida individuals (starved for 2 days) were placed in the center of the petri dish and incubated at $15^{\circ} \mathrm{C}$ in dark. The number of collembolans present on each fungus was counted at different time points for 66 hours. This experiment was carried with four replicates, the experiment repeated at least two times. 


\subsubsection{Choice assay between Fusarium verticillioides, $F$. graminearum and $F$. graminearum mutants}

The same experimental setup as described above was applied. However, in this case $F$. verticillioides (Ita 1), F. graminearum (IFA 66) and F. graminearum deficient mutants $(\triangle \mathrm{DON}, \triangle \mathrm{ZEN}$ and $\triangle \mathrm{AUR}$ ) were offered as food source. Agar plugs (12 mm diameter) with mycelium were placed at the edge of each petri dish. The numbers of collembola visiting each type of mycelia at different time points during a 60-hour period was recorded. Five replicates were carried out, the experiment repeated at least two times. In the next assay, the same conditions and species as before were used. However, F. graminearum $\triangle$ AUR was excluded from the diet.

\subsubsection{Choice assay between Fusarium graminearum, $F$. verticillioides and mixed fungal cultures}

$25 \mu \mathrm{L}$ mixed spore suspension of $F$. graminearum and $F$. verticillioides ( 2 spore $\mu \mathrm{L}$ ) were inoculated on two opposite site of the biomalt maize medium (BMM) and cultured at $20^{\circ} \mathrm{C}$ in dark for 4 days.

Fungal plugs with $F$. graminearum, $F$. verticillioides and mix of themwere harvested from the plate using sterile cork borer (12 mm diameter): Agar plugs with each fungi were transferred to medium with a mixture of plaster of Paris:activated charcoal (9:1). Twenty Folsomia candida, starved for 2 days, were placed at the center of the petri dishes and the number of collembola present on each food source recorded over a 60-hour period.

\subsection{Quantitation of consumed mycelium}

\subsubsection{Food preparation}

Twenty-five $\mu \mathrm{L}$ of $F$. graminearum and $F$. verticillioides spore suspension $(\sim 2$ spores $/ \mu \mathrm{L})$ were inoculated on the opposite ends of BMM medium plates (30 replicates). While $50 \mu \mathrm{L}$ mixed spore suspension of F.graminearum and F.verticillioides (1 germination spore $/ \mu \mathrm{L}$ for each fungal strain) was spread evenly on the BMM medium plates (30 replicates). Plates were cultured at $20^{\circ} \mathrm{C}$ in dark for 4 days.

Ten replicates from each treatment were harvested immediately. To further 10 plates, $30 \pm$ 5 two-day-starved collembolans were added, the remaining 10 treatments were kept free of collembolans. Both treatments were harvested after 9 days. Harvesting was performed by using a sterile scalpel to scrap all the mycelium and transfer it to a $2 \mathrm{~mL}$ Eppendorf tube, 
to which $300 \mu \mathrm{L}$ ethanol was added and evaporated to dryness. After that, they were ground in a Tissuelyzer (MM 200, Retsch, Haan, Germany).

\subsubsection{DNA extraction}

DNA extraction of ball mill-ground mycelia was performed by the CTAB method (Brandfass \& Karlovsky, 2008). DNA quality was checked by electrophoresis in $0.8 \%$ agarose gels stained with ethidium bromide and visualized under UV light by using a digital imaging system (Vilber Lourmat, Eberhardzell, Germany). Chemicals were obtained from Carl Roth GmbH (Karlsruhe, Germany).

\subsubsection{Quantitative real-time PCR}

The primers Fg 16NF /Fg 16NR (Nicholson et al., 1998) and Ver 1 /Ver 2 (Mulè et al., $2004)$ were used to quantify the amounts of $F$. graminearum and $F$. verticillioides. The thermocycler was a CFX384 Real-time system (BioRad Laboratories, München, Germany). For the real time PCR reaction, a total mixture volume of $4 \mu \mathrm{L}$ was used in 384-well microtiter plate (company, city, country). The reaction mixture was comprised of $1 \times$ buffer, $2.5-4.5 \mathrm{mM} \mathrm{MgCl}_{2}, 100 \mu \mathrm{M}-200 \mu \mathrm{M}$ dNTP, $0.3 \mu \mathrm{M}$ of each primer, $0.1 \times$ SYBR Green, $1.0 \mathrm{mg} / \mathrm{mL}$ BSA, and $0.03 \mathrm{U} / \mu \mathrm{L}$ Taq polymerase. The cycling conditions were as follows: initialization at $94^{\circ} \mathrm{C} / 95^{\circ} \mathrm{C}$ for $2 \mathrm{~min}$, denaturation at $94^{\circ} \mathrm{C}$ for $30 \mathrm{sec} / 45$ sec, annealing at $61^{\circ} \mathrm{C} / 62.5^{\circ} \mathrm{C}$ for $30 \mathrm{sec}$, elongation at $68^{\circ} \mathrm{C}$ for $30 \mathrm{sec} / 40 \mathrm{sec}$, melting at $68^{\circ} \mathrm{C}$ for $5 \mathrm{~min} / 4 \mathrm{~min}$ and $95^{\circ} \mathrm{C}$ for $1 \mathrm{~min}$, analysis at $55-90^{\circ} \mathrm{C}$ in $10 \mathrm{sec}$ and $20^{\circ} \mathrm{C}$ for 10 $\min$.

\section{Results}

\subsection{F. candida food preference between $F$. verticillioides and $F$. graminearum}

In all experiments, the collembolans preferred the mycelia of $F$. verticillioides. Almost no springtails were found on $F$. graminearum mycelia (Figure 2).

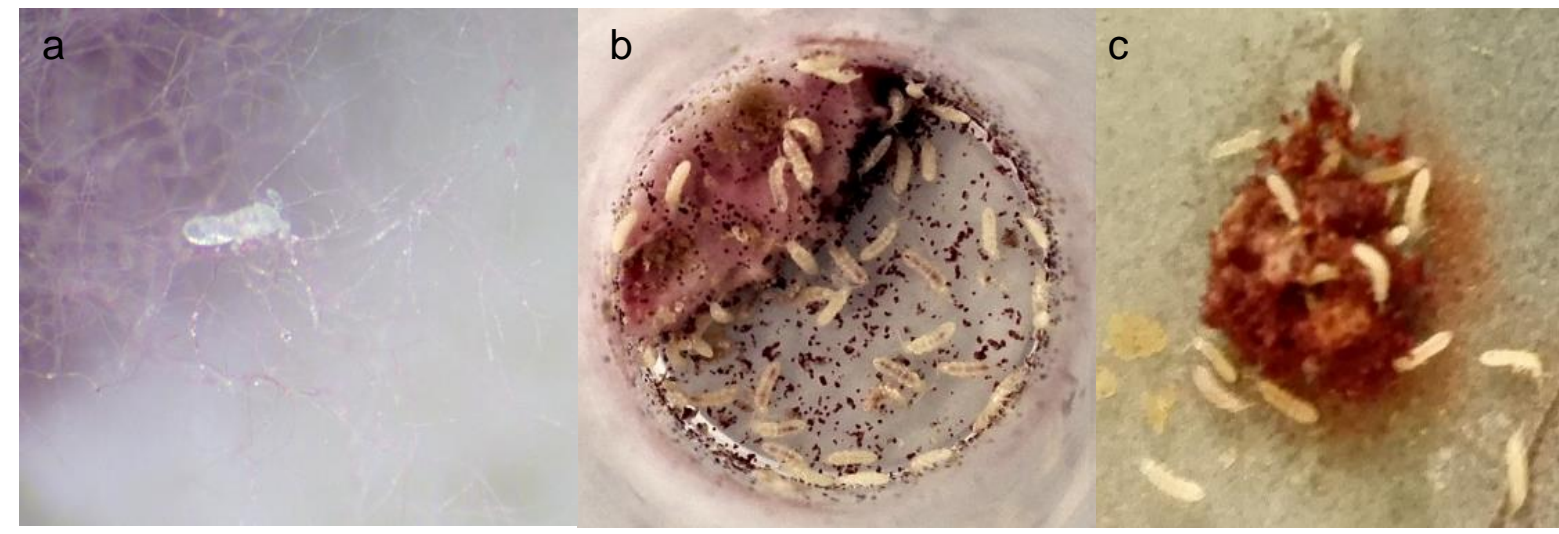


Figure 1: (a) Springtails (F. candida) feeding on Fusarium graminearum mycelium, and induction of red pigment on single mycelia string; (b) Folsomia candida feeding on Fusarium graminearum; (c) collembola are making holes to reach their desirable food ( $F$. verticillioides) in the mixed diet which consists of $F$. graminearum and $F$. verticillioides. Red mycelium is $F$. graminearum and white mycelium is $F$. verticillioides.

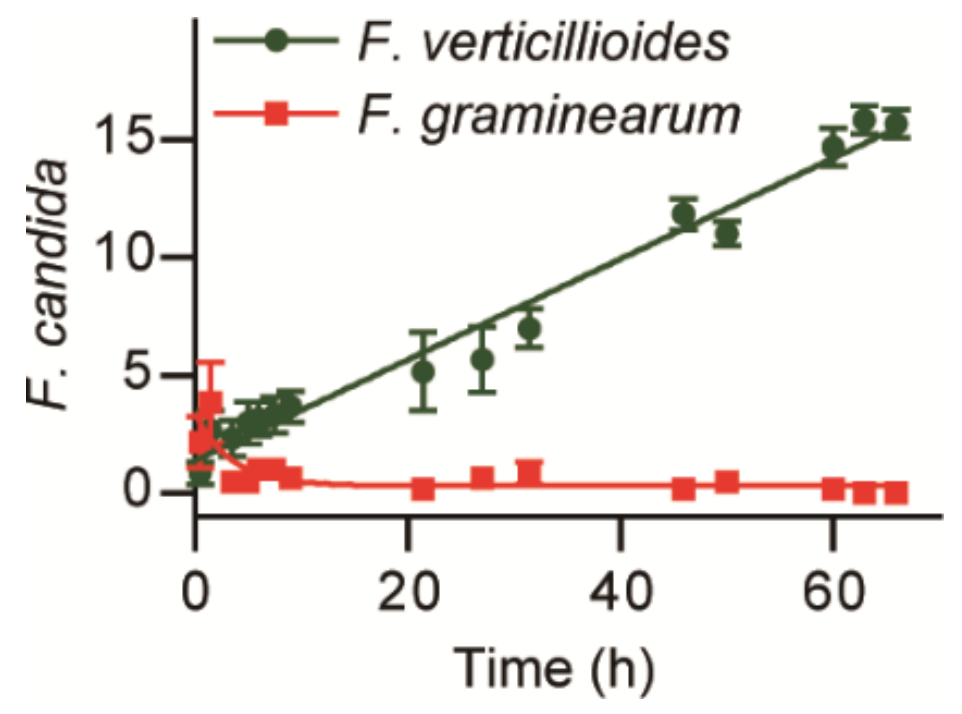

Figure $2 \mid$ Mean number of $F$. candida on mycelia of $F$. verticillioides and $F$. graminearum. Bars represent Mean \pm SE.

\subsection{Food choice of $F$. candida offered $F$. verticillioides and $F$. graminearum mutants impared in secondary metabolite synthesis}

When $F$. verticillioides and $F$. graminearum were offered in a choice assay, $F$. verticilloides was preferred by Folsomia (Figure 2). However, when various F. graminearum mutants $(\triangle \mathrm{AUR}, \triangle \mathrm{DON}, \triangle \mathrm{ZEA})$ were provided as well, collembola only attracted to by $F$. graminearum $\Delta \mathrm{AUR}$. There were no more than five $F$. candida individuals on the mycelia of $F$. verticillioides (Figure 3A). Two or less collembola were present on the mycelia of $F$. graminearum mutants $\triangle \mathrm{DON}$ and $\triangle \mathrm{ZEA}$, even these mutants could not produce the corresponding mycotoxins - deoxynivalenol and tearalenone.

According to above observation, we begin with another similar experiment except for removing of $F$. graminearum $\Delta$ AUR. In this case, most of the arthropods were found on $F$. verticillioides mycelia (Figure 3B), and nearly none of them can be found on the other strains. 
As seen in Figure 2-3, collembolan feeding was very slow at the beginning of the experiment but increased from the 3rd hour. From the 3rd hour till the end of the experiment, significantly more collembola were found on their preferred mycelia.

Fungal strains

$\longrightarrow$ F. verticillioides

- - F. graminearum

$\longrightarrow$ F.graminearum $\triangle \mathrm{DON}$

$\longrightarrow$ F.graminearum $\triangle \mathrm{ZEA}$

$\longrightarrow$ F.graminearum $\triangle \mathrm{AUR}$
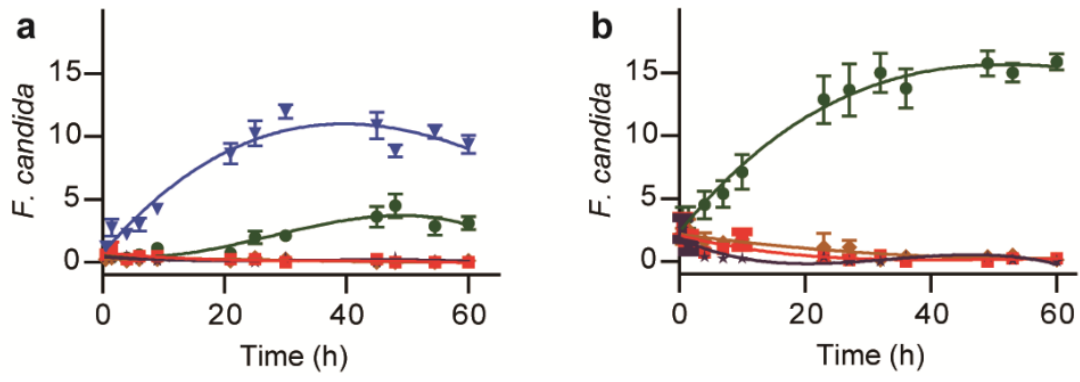

Figure 3 | Multiple choice assays with different fungal strains as food source (a) Folsomia candida prefers the $\triangle$ AUR $F$. graminearum mutant even more than $F$. verticillioides in a multiple choice experiment that also included the totally unattractive wild type of $F$. graminearum; (b) $F$. verticillioides is attractive to collembola, when $\triangle$ AUR $F$. graminearum mutant is absent, the wild type $F$. graminearum and its $\triangle \mathrm{DON}$ and $\triangle \mathrm{ZEN}$ mutants are similarly unattractive.

\subsection{Food preference between $F$. graminearum, $F$. verticillioides, and their mixed culture}

$F$. candida preferred feeding on the mixed diet of $F$. verticillioides and $F$. graminearum compared to single diet of $F$. verticillioides. $F$. graminearum diet was the least attractive for the springtails (Figure 4).

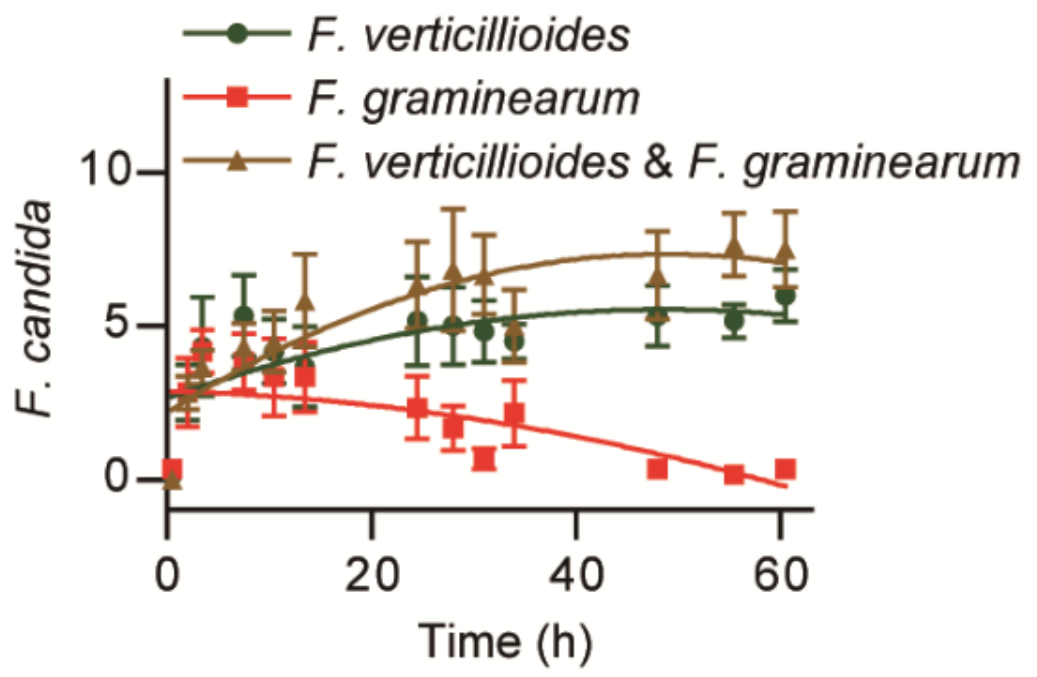

Figure 4: The mean number of $F$. candida recorded on mycelium of $F$. graminearum, $F$. verticillioides and a mix of both fungal species. Bars represent mean $\pm \mathrm{SE}$. 


\subsection{Comparison between food selection and food preference}

According to Figure 5, in the separated assay with animals consuming, mycelia amount of $F$. verticillioides is been largely reduced, and mycelia biomass of $F$. graminearum is induced. And in the mixed assay, $F$. graminearum amount is decrease while the $F$. verticillioides in decline as well. However, in mixed assay, the $F$. verticillioides reduction is not as much as in the separate assay.

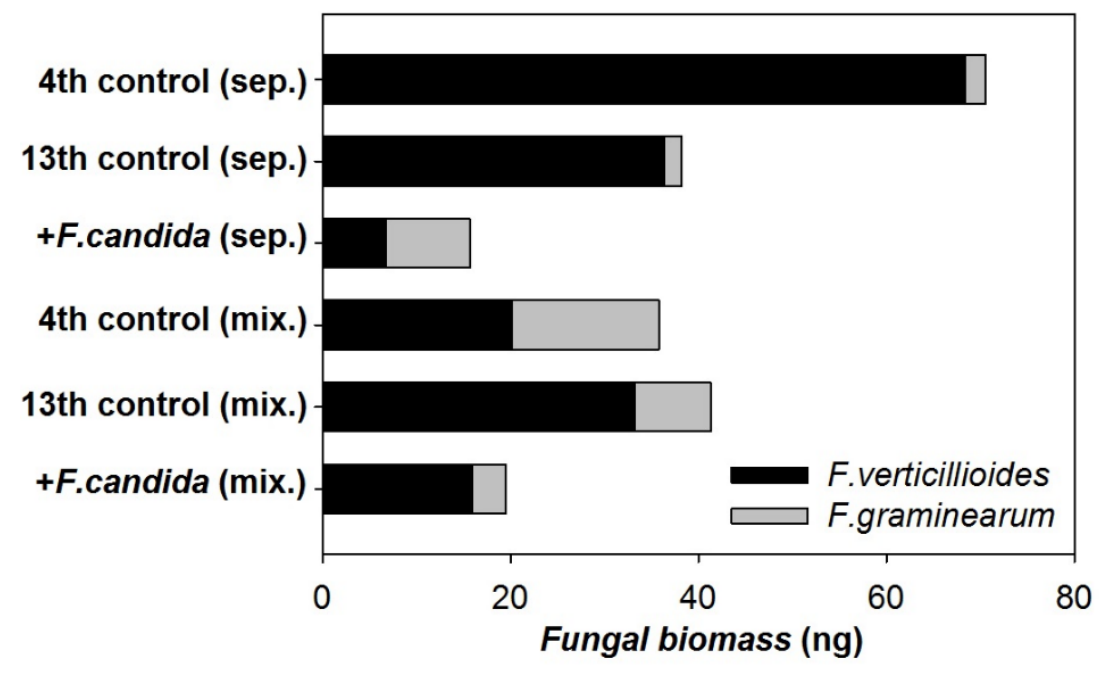

Figure 5. Folsomia candida consumes higher amounts of $F$. graminearum when offered mixed assay (mix.) compared to pure cultures (sep.). Fungal biomass was quantified by real time PCR.

\section{Discussion}

According to the food preference experiment, Fusarium verticillioides turned out to be a preferred food source for springtails, while colonies of $F$. graminearum were avoided (Figure 2). This is consistent with results reported in chapter 2 of this thesis that DNA amount of $F$. verticillioides was significantly reduced after collembolan grazing which was not observed for $F$. graminearum. Documentation exists that Fusarium verticillioides promotes growth of invertebrates in laboratory and field assays (Schulthess et al., 2001; Cardwell et al., 2000). In addition, Munkvold (2003) pointed out that insect disperse $F$. verticillioides more efficiently than $F$. graminearum, which agrees with the observed and known results.

Even though $F$. graminearum and $F$. verticillioides belong to the same genus, they differentially attract collembola (Figure 2). Nevertheless, collembola prefer the $\triangle$ AUR $F$. 
graminearum, the mutant that lacks the biosynthetic pathway that producing aurofusarin. This indicates that aurofusarin protects the fungus from predation. In our study, however, the tested mutants derive from different $F$. graminearum wild-type strains, which, to some extent, could have affected the results. At least, $\triangle \mathrm{AUR}$ and $\triangle \mathrm{ZEN} F$. graminearum share the same wildtype.

Several studies demonstrated that fungal secondary metabolites deter invertebrates. Döll et al. (2013) reported that F candida preferred ungrazed to grazed Aspergillus nidulans. Secondary metabolites, such as austinol, dehydroaustinol, emericellamide and sterigmatocystin, were induced after grazing. A. nidulans $\Delta$ lae which is in deficient of producing various secondary metabolites, was consumed in higher amounts than the wild type strain (Rohlfs et al., 2007). $\triangle P k s P$ A. fumigatus, whose melanin biosynthesis pathway had been disrupted, was more attractive to collembola (Scheu and Folger, 2004).

Generally, F. candida preferred mixed species to single species mycelium. This is consistent with Scheu \& Folger (2004) who suggested collembolans benefit from mixed diet as a consequence of better reproduction and increased fitness. When the animals fed on a diet comprising a mixture of $F$. graminearum and $F$. verticillioides, $F$. candida still preferred $F$. verticillioides, but the consumption of $F$. graminearum was much higher under conditions of mixed colonies. This might be because collembola are not able to distinguish their preferred diet as well when the Fusarium spp. were offered separately.

According to Picot et al. (2012), F. verticillioides can inhibit the growth of co-cultured $F$. graminearum. Even if the $F$. graminearum infection was higher on maize, $F$. verticillioides could still out-compete it. In this study, with the addition of $F$. candida whose preferred food choice is $F$. verticillioides, the dominance of $F$. verticillioides is affected. Although in the mixed diet is still competitively dominant.

Our results with mixed cultures indicate that traditional food choice experiments with spatially separated colonies of fungal isolates do not adequately reflect the behavior of frugivorous arthropods in natural ecosystems. Crowther et al. (2012) reported that, compared to collembolans, isopods ware more aggressive feeders. This supports the notion that feeding experiments should therefore involve co-cultures of several fungal species as well as different animal species. 


\section{References}

Bardgett, R.D., Whittaker, J.B. and Frankland, J.C. (1993). The diet and food preferences of Onychiurus procampatus (Collembola) from up land grassland soils. Biology and Fertility of Soils 16: 296-298.

Bengtsson, G., Hedlund, K. and Rundgren, S. (1991). Selective odor perception in the soil collembola Onychiurus armatus. Journal of Chemical Ecology 17: 2113-2125.

Brandfass, C. and Karlovsky, P. (2008). Upscaled CTAB-based DNA extraction and realtime PCR assays for Fusarium culmorum and F.graminearum DNA in plant material with reduced sampling error. International Journal of Molecular Sciences 9: 2306-2321.

Crowther, T.W., Boddy, L. and Jones, T.H. (2012). Functional and ecological consequences of saprotrophic fungus-grazer interactions. The ISME Journal 6: $1992-2001$

Crowther, T.W. and Donald, A'Bear. (2012). Impacts of grazing soil fauna on decomposer fungi are species-specific and density-dependent. Fungal Ecology 5: 277-281.

Döll, K., Chatterjee, S., Scheu, S., Karlovsky, P. and Rohlf, M. (2013). Fungal metabolic plasticity and sexual development mediate induced resistance to arthropod fungivory. Proceedings of the Royal Society B: Biological Sciences 280: 20131219

Hopkin, S.P. (1997). Biology of the Springtails (Insecta: Collembola). Oxford University Press, Oxford.

Innocenti, G., Sabatini, M.A., Ganassi, S., Montanari, M. and Branzanti, M.B. (2009). Trophic interactions between soil fungi and collembola. Multitrophic interactions in soil. IOBC Bulletin 42: 91-94.

Jonas, J.L., Wilson, G.W.T., White, P.M. and Joern, A. (2007). Consumption of mycorrhizal and saprophytic fungi by Collembola in grassland soils. Soil Biology \& Biochemistry 39: 2594-2602.

Jorgensen, H.B., Elmholt, S. and Petrsen, H. (2003). Collembola dietary specialisation on soil grown fungi. Biology and Fertility of Soils 39: 9-15. 
Kaneda, S. and Kaneko, N. (2004). The feeding preference of a collembolan (Folsomia candida Willem) on ectomycorrhiza (Pisolithus tinctorius (Pers.)) varies with mycelial growth condition and vitality. Applied Soil Ecology 27: 1-5.

Maier, F. J., Miedaner, T., Hadeler, B., Felk, A., Salomon, S., Lemmens, M., Kassner, H. and Schäfer, W. (2006). Involvement of trichothecenes in fusarioses of wheat, barley and maize evaluated by gene disruption of the trichodiene synthase (Tri5) gene in three field isolates of different chemotype and virulence. Molecular Plant Pathology 7: 449461.

Malz, S., Grell, M.N., Thrane C., Maier, F.J., Rosager, P., Felk, A., Albertsen, K.S., Salomon, S., Bohn, L., Schäfer, W. and Giese, H. (2005). Identification of a gene cluster responsible for the biosynthesis of aurofusarin in the Fusarium graminearum species complex. Fungal Genetics and Biology 42: 420-433.

Marin, S., Ramos, A.J., Cuevas, D. and Sanchis V. (2006). Fusarium verticillioides and Fusarium graminearum infection and fumonisin B1 and zearalenone accumulation in resveratrol-treated corn. Food Science Technology International 12: 353-359.

Miedaner, T., Bolduan, C. and Melchinger, A.E. (2010). Aggressiveness and mycotoxin production of eight isolates each of Fusarium graminearum and Fusarium vertillioides for ear rot on susceptible and resistant early maize. European Journal of Plant Pathology 127: 113-123.

Mule, G, Susca, A, Stea, G, Moretti, A. (2004). A species-specific PCR assay based on the calmodulin partial gene for the identification of Fusarium verticillioides, $F$. proliferatum and F. subglutinans. European Journal of Plant Pathology 110: 495502.

Munkvold, G.P. (2003). Epidemiology of Fusarium diseases and their mycotoxins in maize ears. European Journal of Plant Pathology. 109: 705-713.

Nicholson, P., Simpson, D.R., Weston, G., Rezanoor, H.N., Lees, A.K., Parry, D.W. and Joyce, D. (1998). Detection and quantification of Fusarium culmorum and Fusarium graminearumin cereals using PCR assays. Physiological and Molecular Plant Pathology 53: 17-37.

Picot, A., Hourcade-Marcolla, D., Barreau, C., Pinson-Gadais, L., Caron, D., RichardForget, F. and Lannou, C. (2012). Interactions between Fusarium verticillioides 
and Fusarium graminearum in maize ears and consequences for fungal development and mycotoxin accumulate. Plant Pathology 61: 140-151.

Ponge, J.F. (2000). Vertical distribution of Collembola (Hexapoda) and their food resources in organic horizons of beech forests. Biology and Fertility of Soils 32: $508-522$.

Rohlfs, M., Albert, M., Keller, N.P. and Kempken, F. (2007). Secondary chemicals protect mould from fungivory. Biology Letters 3:523-525.

Scheu, S. and Folger, M. (2004). Single and mixed diets in collembolan: effects on reproduction and stable isotope fractionation. Functional Ecology 18: 94-102.

Schneider, K. and Maraun M. (2005). Feeding preferences among dark pigmented fungal taxa ("Dematiacea") indicate limited trophic niche differentiation of oribatid mites (Oribatida, Acari). Pedobiologia 49: 61-67.

Schulthess, F., Cardwell, F. and Gounou, S. (2001). The effect of endophytic Fusarium vertcillioides on infestation of two maize varieties by lepidopterous stemborers and coleopteran grain feeders. Phytopathology 92: 120-128.

Varga, J., Naar, Z. and Dobolyi, C. (2002). Selective feeding of collembolan species Tomocerus longicornis (Müll.) and Orchesella cincta (L.) on moss inhabiting fungi. Pedobiologia 46: 526-538. 


\title{
Chapter 4: Nontoxic naphthopyrone pigment protects filamentous fungi from a wide range of animal predators
}

\author{
Yang Xu, Maria Vinas, Albatol Alsarrag, Ling Su, Katharina Pfohl, \\ Wei Chen, Petr Karlovsky*
}

\section{Main text}

Soil fungi play a key role in nutrient cycling by degrading recalcitrant plant biomass (McGonigle 2007). Predators feeding on fungi influcence fungal network architecture (Boddy et al. 2010) and modulate the composition (McGonigle 2007) and activity (A'Bear et al. 2014) of fungal communities, affecting their contribution to ecosystem services (Crowther et al. 2012). As a defence against predators, fungi produce toxic proteins and metabolites. The role of lectins and other proteins in fungal defence have recently been established (Schubert 2012, Künzler 2015, Wohlschlager 2014, Plaza et al. 2016) but evidence for defence metabolites remains inconclusive, though efforts to establish the role of mycotoxins in fungal defence date three decades back (Wicklow 1988, Dowd 1992). Here we show that nontoxic pigment aurofusarin, rather than mycotoxins, protects fungi from a range of phylogenetically distant predators. Springtails (collembolans), woodlice (crustaceans) and mealworms (insects) avoided cultures of fungus Fusarium graminearum accumulating aurofusarin and mealworms were repelled by wheat flour amended with aurofusarin. Transcription of aurofusarin synthesis genes was stimulated by feeding of springtails, woodlice and nematodes, causing accumulation of high amount of aurofusarin in mycelia ( $>1 \%$ dry weight). Forced-feeding on fungi accumulating aurofusarin did not cause mortality of springtails and woodlice and purified aurofusarin exerted low toxicity to mealworms and insect cells. Thus contrary to previous views (Wicklow 1988, Dowd 1992, Rohlfs 2015) a nontoxic antifeedant rather than mycotoxins protected $F$. graminearum from a wide range of animal predators. Bis-naphthopyrone pigments similar to aurofusarin are ubiquitous among filamentous Ascomycota. Together with the magnitude of the repellent effect of aurofusarin and its consistency across diverse predators this suggests that bis-naphthopyrone pigments are major defence metabolites of Ascomycota against grazing invertebrates. 
The role of mycotoxins in defence of fungi against predation by has been proposed decades ago and many mycotoxins were shown to possess insecticidal activities (Wicklow 1988, Dowd 1989, Dowd 1992) but efforts to prove their defence function in ecologically relevant context remained largely inconclusive (Ruess \& Lussenhop, 2005; Rohlfs 2015). Circumstantial support for the defence role of mycotoxins was provided by stimulation of mycotoxin synthesis by arthropod grazing (Döll 2013) and mechanical injury (Brandt et al. 2017) and by accumulation of toxic metabolites in reproductive structures and sclerotia (Calvo \& Cary 2015). Challenging arthropods with fungal strains impaired (Rohlfs et al. 2007, Trienens et al. 2010) and constitutively stimulated (Yin et al. 2012) in secondary metabolism generated encouraging results but pleiotropic character of these mutations prevented assignment of effects to causes. Mycotoxin sterigmatocystin was most often implicated in defence but pathway-specific mutants failed to confirm its role (Rohlfs 2015). The only mycotoxins proven to act as defence metabolites are asparasone protecting sclerotia of Aspergillus flavus from sap beetles (Cary et al. 2014) and gliotoxin protecting A. flavus from soil amoeba (Hillmann 2015). Despite extensive efforts, the role of other mycotoxins in defence has not been substantiated.

Assuming that defence metabolites are synthesized on demand, transcriptome of fungus Fusarium graminearum exposed to springtail Folsomia candida was investigated to reveal which pathways were induced by grazing. RNA was extracted from fungal cultures subjected to grazing and intact cultures and mRNA levels for all genes were compared by RNA sequencing (RNAseq). Grazing stimulated transcription of pathways for metabolites aurofusarin, fusarin $\mathrm{C}$ and fusaristatin A (Fig. 1), several gene clusters putatively involved in secondary metabolite synthesis (Extended Data Fig. 1), and seven genes encoding small cystein-rich proteins (Extended Data Fig. 2). 


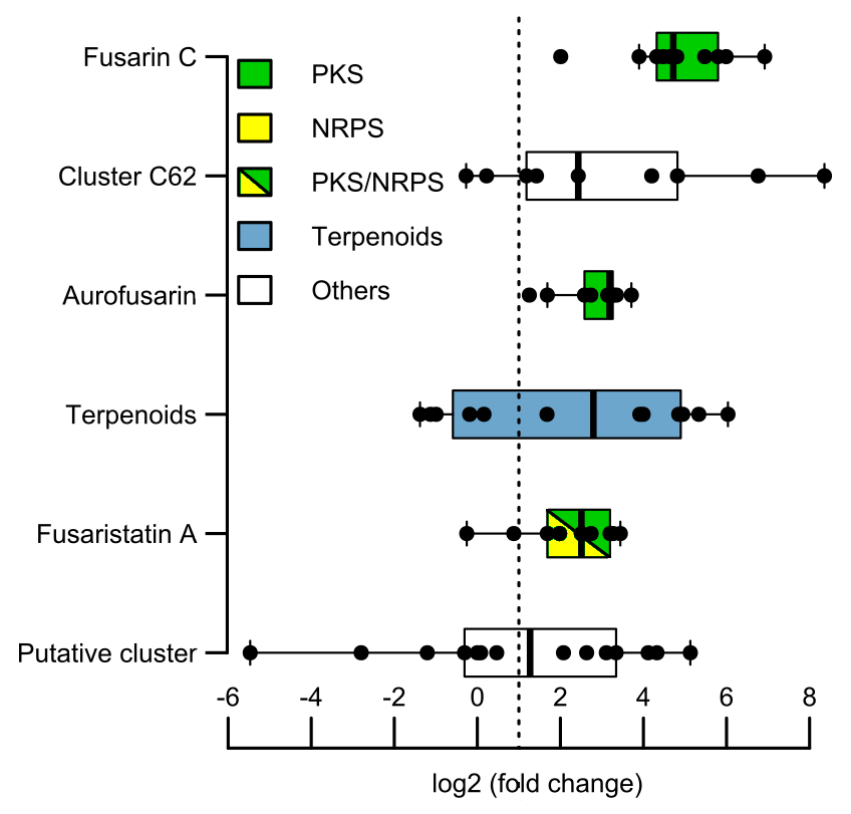

Figure 1 | Gene clusters for secondary metabolites in Fusarium graminearum up-regulated by springtail grazing. F. graminearum culture was exposed to springtail Folsomia candida for 48 hours. RNA was extracted and mRNA levels were determined by RNA sequencing (RNAseq) and compared. Black points show log2(fold change) values for each gene. Up-regulated clusters were identified based on the criteria that more than $50 \%$ of the genes in the cluster and/or the gene encoding a signatures enzyme were significantly induced [ $\log 2$ FPKM (fold change) higher than 1.0 (dotted line) and q value was lower than 0.01]. Accession numbers: fusarin C (FGSG_07798, FGSG_07800 to FGSG_07805, FGSG_13222 to FGSG_13224), cluster C62 (FGSG_10606, FGSG_10608, FGSG_10609, FGSG_10611 to FGSG_10614, FGSG_10616, FGSG_10617), aurofusarin (FGSG_02320 to FGSG_02329), terpenoids (FGSG_01737 to FGSG_01749), fusaristatin A (FGSG_08204 to FGSG_08210, FGSG_08213, FGSG_08214), putative cluster (FGSG_10557 to FGSG_10560, FGSG_10562 to FGSG_10567, FGSG_10569 to FGSG_10571, FGSG_10573). RNAseq analysis was carried out on four biological replicates. Boxplots show the median for each cluster and lower and upper quartiles (Q1 and Q3). Whiskers show the largest (smallest) observation or 1.5-fold of the interquartile range, whichever is smaller (larger) [Q1$1.5 *(\mathrm{Q} 3-\mathrm{Q} 1)$ and $\mathrm{Q} 3+1.5 *(\mathrm{Q} 3-\mathrm{Q} 1)]$. 
We selected aurofusarin for further work because it is produced by many fungal species belonging to several genera (Malz et al. 2005; Põldmaa 2011). Aurofusarin is a dimeric naphtho- $\gamma$-pyrone (Fig. 2f) causing colorizing of maize cobs infected with $F$. graminearum (Fig. 2a). All genes of the aurofusarin biosynthetic cluster except one were induced by springtail grazing (Fig. 2c). To examine whether aurofusarin is also induced by other predators, cultures of $F$. graminearum and $F$. culmorum were subjected to springtail $F$. candida and fungivorous nematodes Aphelenchoides saprophilus and Bursaphelenchus mucronatus for different time periods, fungal RNA was extracted and relative mRNA levels for five genes of aurofusarin synthesis were estimated using RT qPCR (Fig. $2 \mathrm{j}$ to k). All five genes of aurofusarin pathway were strongly induced in both Fusarium species 48 $\mathrm{h}$ after exposure to springtails and $24 \mathrm{~h}$ after exposure to nematodes. Aurofusarin was extracted from mycelia of both fungi exposed to springtail grazing and its content was estimated by HPLC (Fig. 2d, e). In line with the induction of gene transcription, strong increase of aurofusarin levels was found in grazed culture as compared to controls in both fungi. In axenic cultures, production of aurofusarin in F. graminearum was stimulated by shaking (Fig. 2b). We assume that mechanical damage caused by shaking triggered defence responses that are normally activated by predation.

To find out whether aurofusarin synthesis is induced by grazing in other Fusarium species, cultures of $F$. poae, $F$. venenatum and $F$. avenaceum growing on agar media were subjected to feeding by spring tail Folsomia candida and F. venenatum and F. sporotrichioides were subjected to grazing by woodlice Porcellio scaber. Mycelia affected by grazing turned red, indicating that feeding damage stimulated aurofusarin synthesis in all four Fusarium species (Extended Data Fig. 3).

The key criterion for the involvement of a metabolite in defence against predation is that it reduces feeding damage. To test whether aurofusarin protected its producers from predation, food choice experiments with a F. graminearum strain accumulating aurofusarin and isogenic gene-disruption mutants unable to synthesize aurofusarin were carried out. Three independently obtained mutants were used to exclude effects of unaccounted for changes in the genome during gene disruption. Predators representing phylogenetically distant arthropod lineages were used: collembolan $F$. candida (Entognatha), woodlice Trichorhina tomentosa (Crustacea) and mealworm Tenebrio molitor (Insecta). Cultures of aurofusarin-producing and non-producing strains were offered to the predators on opposite sides of Petri dishes and the number of animals on or 

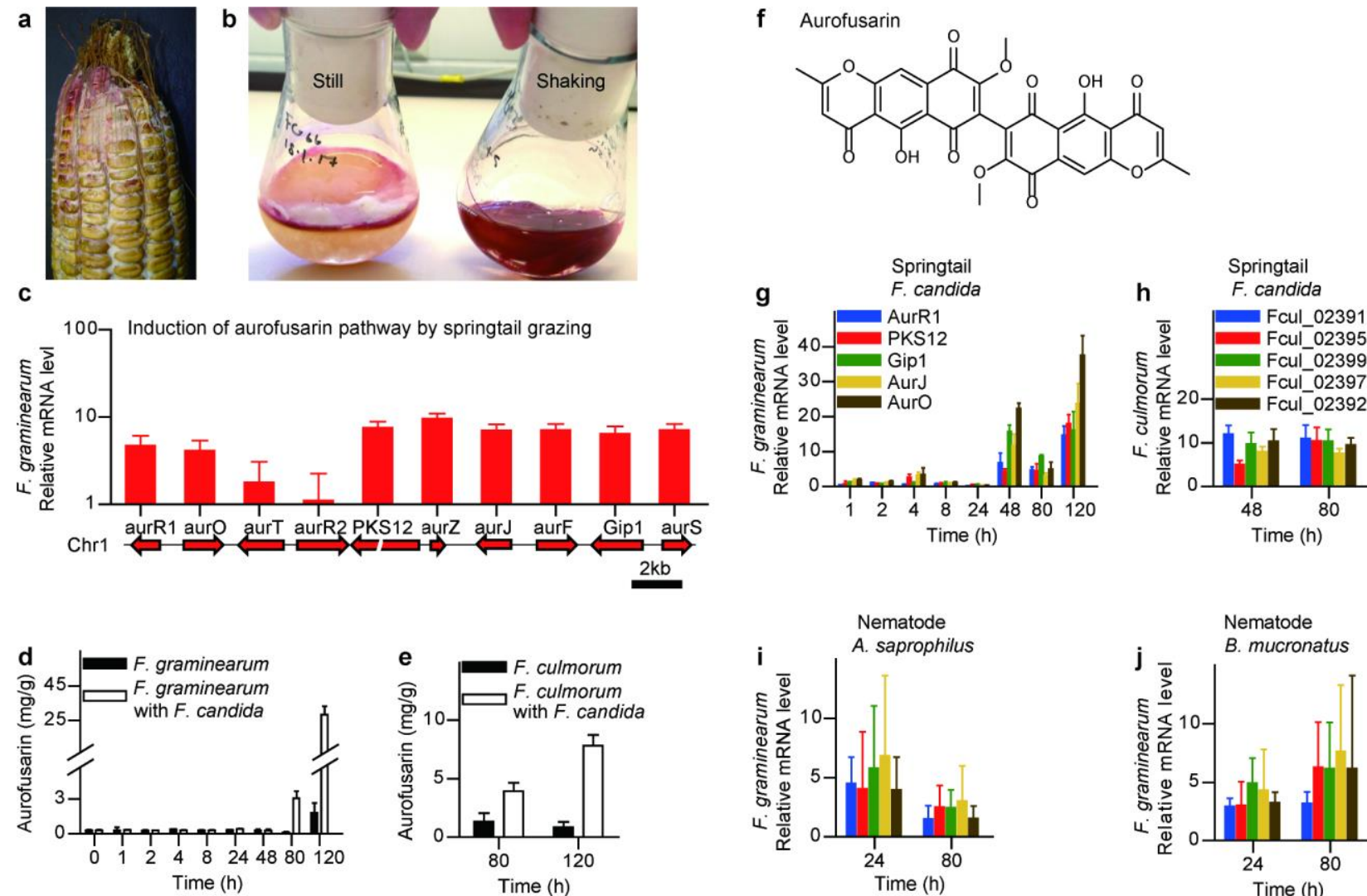

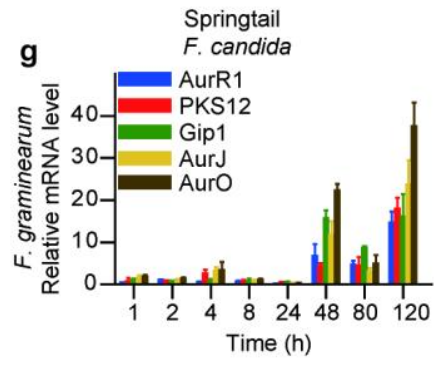

f Aurofusarin<smiles>COC1=C(C2=C(OC)C(=O)c3cc4oc(C)cc(=O)c4c(O)c3C2=O)C(=O)c2c(cc3oc(C)cc(=O)c3c2O)C1=O</smiles>

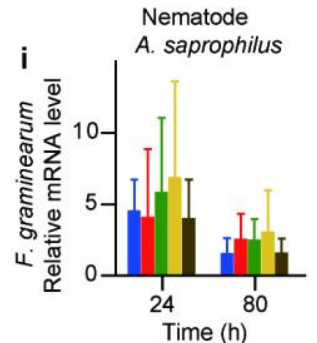

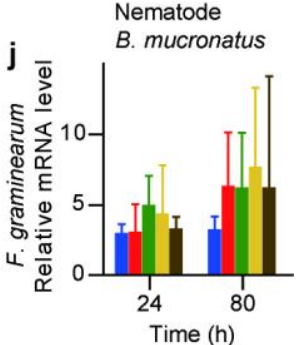

Figure 2 Aurofusarin synthesis in $F$. graminearum and $F$. culmorum is stimulated by springtail and nematode feeding. a, Corn ear inoculated with $F$. graminearum (photo by courtesy of Dr. Belinda J. van Rensburg, ARC South Africa). b, F. graminearum cultures in potato infusion broth with and without shaking for two weeks. c, Up-regulation of genes of aurofusarin biosynthetic in $F$. graminearum after exposure to grazing by springtail $F$. candida for $48 \mathrm{~h}$ (RNAseq, see Fig. 1). d,e, Time course of aurofusarin accumulation in F. graminearum and $F$. culmorum cultures exposed to grazing by springtail $F$. candida. f, Molecular structure of aurofusarin. g,h, Time course of the up-regulation of genes of aurofusarin synthesis in $F$. graminearum and $F$. culmorum after grazing by $F$. candida (RT qPCR). i,j, Up-regulation of genes of aurofusarin synthesis after exposure to fungivorous nematodes A. saprophilus and $B$. mucronatus (RT qPCR). Means are shown as bars with whiskers showing standard error. Three to four biological replicates were used in all experiments. 
in the vicinity of each culture was recorded for $8 \mathrm{~h}$ (springtails and woodlice) or $12 \mathrm{~h}$ (mealworms). All three predators exhibited a strong preference for aurofusarinnonproducing mutants (Fig. 3). For comparison, disruption of biosynthetic pathways for mycotoxins deoxynivalenol and zearalenone in F. graminearum has not affected food choice of the animals (Chapter 3) though both mycotoxins were shown to be toxic to caterpillars (Dowd, Miller and Greenhalgh, 1989; Dowd 1992).

Disruption of aurofusarin pathway may have indirectly affected the synthesis of other metabolites. Can the preference of arthropods for aurofusarin mutants be caused by these differences, rather than by the lack of aurofusarin? Preference of mealworms for wheat flour with and without aurofusarin was investigated to answer this question (Fig. 4e). Strong preference of the larvae for unamended flour confirmed that aurofusarin acted as an antifeedant. The magnitude of the deterrence of predators by aurofusarin-containing cultures indicated that aurofusarin was the major-if not the only-defence compound responsible for the deterrence of predators by F. graminearum in these experiments (Fig. $3)$.

If aurofusarin is toxic, the ability of arthropods to sense and avoid aurofusarin-containing food may have developed as an adaptation reducing their exposure to the toxin. No data on toxicity of aurofusarin to animals are available. Toxicity of aurofusarin to poultry was reported (Dvorska et al. 2001) but because an aurofusarin-containing fungal culture rather than purified aurofusarin was used in this work, the animals were likely exposed to mycotoxins not accounted for in the study. To determine whether aurofusarin is toxic to arthropods, springtail F. candida and isopod T. tomentosa were fed on fungal cultures accumulating aurofusarin and on aurofusarin-free mutants for 5 weeks. Mortality of animals was monitored and the length and width of their bodies were determined at the end of the experiment (Fig. 4a, b). All animals were alive at the end of the experiment. Both arthropods grew faster on aurofusarin-nonproducing mutants. The differences were significant but small; we therefore assume that growth suppression on aurofusarincontaining cultures was caused by reduced food consumption rather than by toxicity. Toxicity of aurofusarin to insects was studied by feeding mealworms on wheat flower amended with purified aurofusarin for $10 \mathrm{~d}$ and comparing the weight gain of the larvae on flour with and without aurofusarin (Fig. 4c). No effect of aurofusarin on mealworms 
a
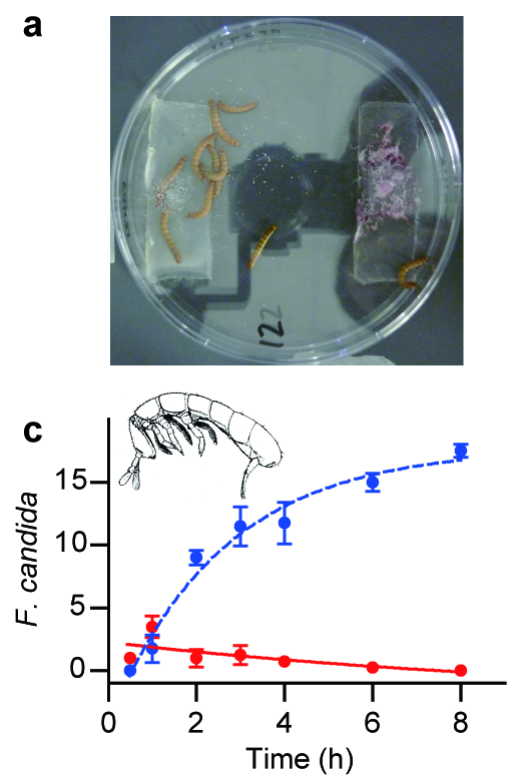

e
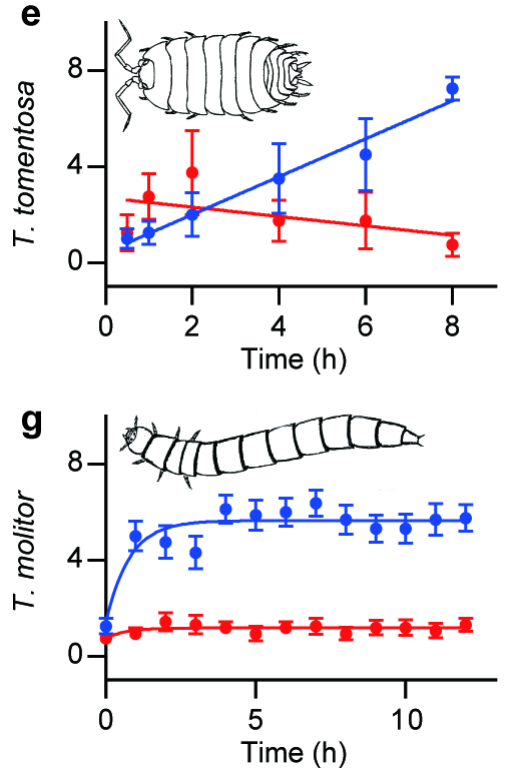
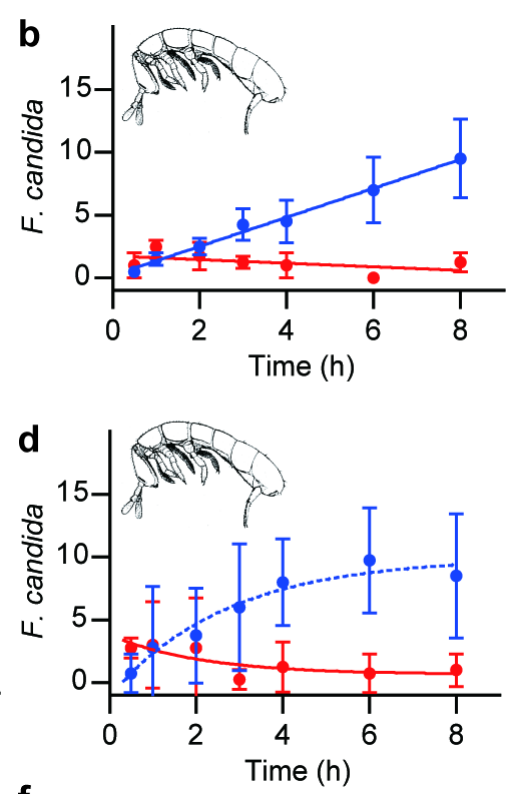

f

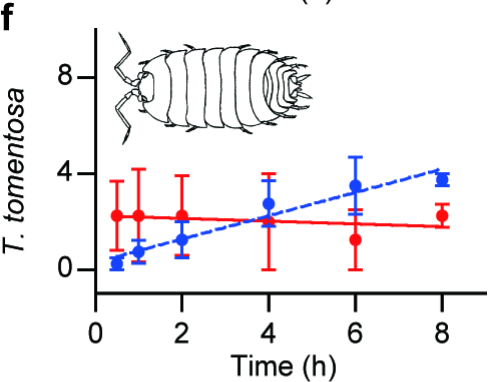

$\longrightarrow \quad$ F. graminearum WT

$\longrightarrow \quad$ F. graminearum $\triangle$ AUR1

---- F. graminearum $\triangle \mathrm{AUR} 2$

---•.. Framinearum $\triangle$ AUR3

Figure 3 | Predators avoid fungal cultures accumulating aurofusarin. a, Mealworms in a Petri dish with cultures of $F$. graminearum accumulating aurofusarin (WT, right) and aurofusarinnonproducing mutant ( $\triangle \mathrm{AUR}$, left). b-d, Number of $F$. candida individuals on $F$. graminearum WT and $\triangle$ AUR mutants. Springtails starved for two days were placed into the center of Petri dish containing fungal cultures and animals on each mycelium were counted (20 animals per plate; 4 replicates). e,f, Food preference of isopod T. tomentosa was tested in the same way with 8 animals per arena and 4 replicates. g, Food choice of mealworm T. molitor was examined by placing ten individuals in a Petri dish containing fungal cultures on microscope slides placed into opposite sides of the dish. The number of animals visiting each culture was recorded by taking photos and counting animals inside rectangles $13 \times 6 \mathrm{~cm}$ drawn around each slide. Ten animals per plate with 16 replicates were used. Bars represent 95\% CI. 

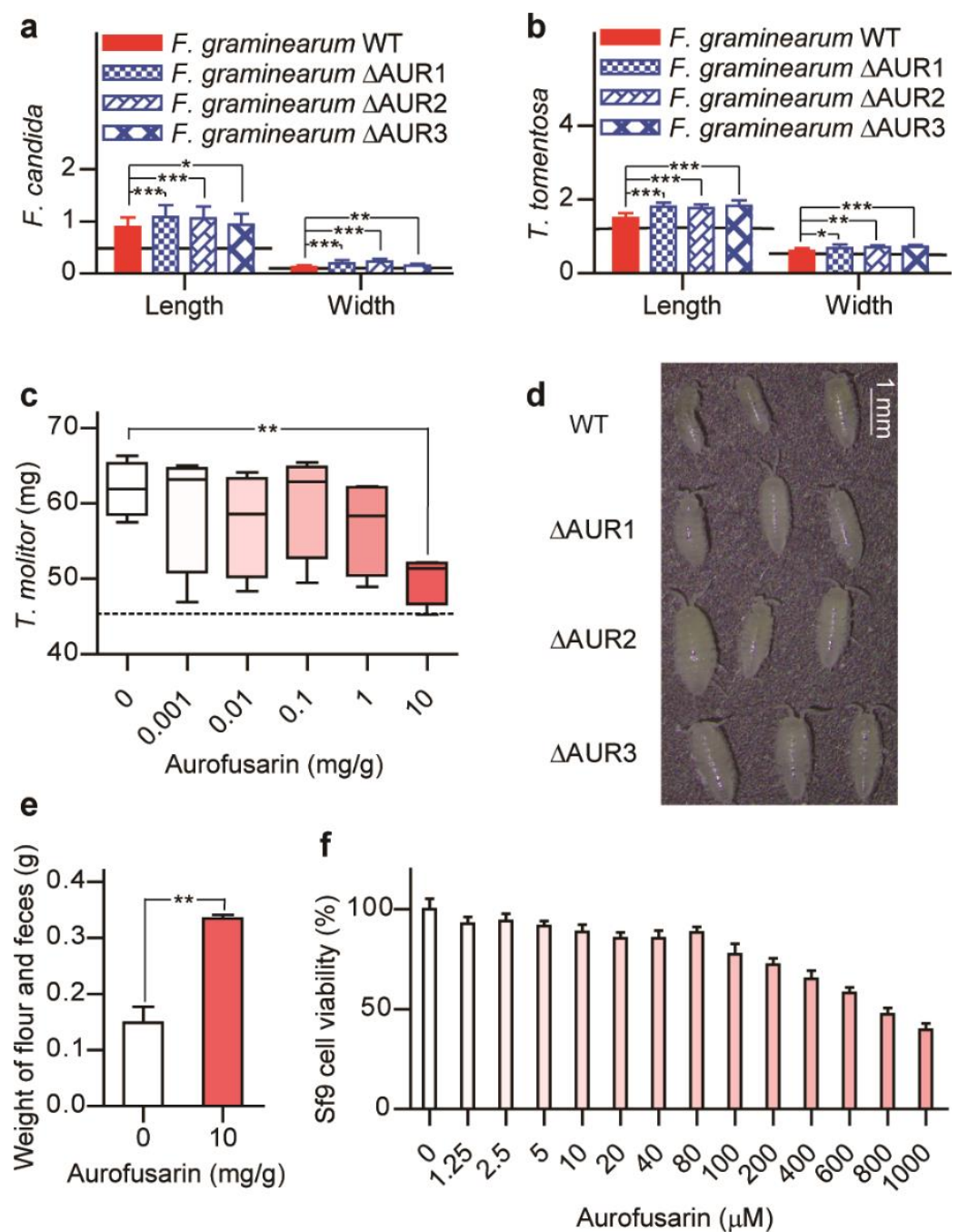

Figure $4 \mid$ Aurofusarin is not toxic to arthropods. a, b, Length and width of $F$. candida and $T$. tomentosa after feeding on $F$. graminearum and three aurofusarin-deficient mutants. Ten $F$. candida or five $T$. tomentosa individuals were placed into Petri dishes with fungal cultures. Animals' length and width were measured at the beginning (horizontal lines) and after 5 weeks. Significance of differences between means was tested by un-paired t-test $(n=4, * 0.05<\mathrm{P}<0.01$, $* * 0.01<\mathrm{P}<0.0001, * * * \mathrm{P}<0.0001)$. c, The weight of $T$. molitor (10 individuals) after 10 days feeding on wheat amended with aurofusarin. Dashed line indicates the average of the initial weight. Box plots show lower and upper quartiles (Q1 and Q3) and the median with whiskers for the smallest and largest values. Data were analysed by un-paired t-test $(\mathrm{n}=4, \mathrm{p}=0.0027)$. d, $T$. tomentosa growth status after feeding on each fungal diet for five weeks. e, Weight of wheat flour and faeces left by T. molitor larvae after $4 \mathrm{~d}$ feeding on unamended wheat flour and flour containing $10 \mathrm{mg} / \mathrm{g}$ aurofusarin. Significance of the difference was analysed by un-paired t-test $(n=3, p=0.0033)$. f, Sf9 cell viability after 24 hours incubation with different concentrations of aurofusarin, $\mathrm{Sf} 9$ cells were seeded into 96 -well cell culture plates at $5 \times 10^{3}$ and $8 \times 10^{3}$ cells per well respectively $(n=3)$. Bars represent means with standard errors. 
was apparent up to $1 \mathrm{mg}$ aurofusarin per $\mathrm{g}$ flour. This concentration is two orders of magnitude higher that concentrations at which typical mycotoxins exert toxicity to insects (Dowd at al., 1989; Dowd 1992). Growth suppression was observed on flour with a very high concentration of aurofusarin (Fig. 4c). It should be noticed that aurofusarin content in fungal mycelia and especially in mycelia damaged by grazing (Fig. 2i) reached even higher concentrations.

Relatively low toxicity of aurofusarin to arthropods can be accounted for by inefficient absorption, detoxification in the digestion track, or by fast clearance. Toxicity assays with cell cultures circumvent these effects. We therefore investigated the effect of aurofusarin on cell culture of fall armyworm (Spodoptera frugiperda), which served as a model for toxicity of mycotoxins to insects (Dowd at al., 1992; Dowd et al., 1989). Aurofusarin exhibited very low toxicity to insect cells (Fig. 4f); concentrations higher than $800 \mu \mathrm{g} / \mathrm{ml}$ were needed for the inhibition of tetrazolium-reducing exceeding 50\% (Fig. 4f). Low solubility of aurofusarin prevented us from recording the entire dose-response curve.

Low toxicity of aurofusarin to arthropods contradicts the current view that metabolites of fungal defence against predators are mycotoxins. The magnitude of the deterrence effect exhibited by aurofusarin and its efficacy against phylogenetically distant arthropods indicate that dimeric naphthopyrones are major defence metabolites of filamentous fungi. This may explain why attempts to substantiate the role of mycotoxins in defence against arthropods remained inconclusive in spite of decades-long efforts. While traditional mycotoxins comprise metabolites of extraordinary chemical diversity and each mycotoxin is produced by a single or few fungal species, aurofusarin is produced by many species of several genera, which inhabit temperate as well as tropical climates (Extended Data Fig. 4). Dimeric naphthopyrones similar to aurofusarin are produced by many genera of filamentous Ascomycetes (Extended Data Fig. 4). Viomellein was selected to determine whether our findings can be extended to dimeric naphthopyrones different from aurofusarin. Cultures of viomellein producer Aspergillus ochraceus were subjected to grazing of springtail $F$. candida and mealworm T. molitor and viomellein concentration was determined by HPLC. Grazing of both arthropods stimulated the accumulation of viomellein (Extended Data Fig. 5), indicating that viomellein may act as a defence metabolite, too. Food-choice experiments will be needed to determine whether viomellein deters arthropods from feeding. 
The concentration of aurofusarin in fungal mycelia exceeds concentrations of typical mycotoxins by several orders of magnitude (Fig. 2e). Oh the other hand aurofusarin synthesis incurs substantial fitness costs, as evident from markedly increased growth rate and conidia production of aurofusarin-nonproducing mutants (Malz et al. 2005). We speculate that high concentrations of aurofusarin in mycelia prevented adaptation of predators to its antifeedant effect. Insects are notorious for rapid development of resistance to insecticides due to the ability of their P450 oxidases to adapt to new substrates (Scott 1999). High concentrations of aurofusarin and other dimeric naphthopyrones in fungi may saturate detoxification activities or other compensatory changes in arthropods, while accumulation of mycotoxins to comparably high concentrations would severely reduce the fitness of their producers by self-poisoning. Synthesis of nontoxic defence chemical at very large amounts typifies a new concept in fungal ecological chemistry with aurofusarin serving the first example. Re-focusing from traditional mycotoxins to naphthopyrone pigments opens new perspective for research in fungal chemical defence and chemical ecology of soil fauna.

\section{Materials and Methods}

\subsection{Animals}

Culture of springtail Folsomia candida (Hexapoda: Collembola) was obtained from Dr. Marko Rohlfs (University of Goettingen, Germany). Larvae of beetle Tenebrio molitor (Insecta: Coleoptera) and isopod Trichorhina tomentosa (Crustacea: Oniscidea) were purchased from Zoo \& Co. Zoo-Busch and b.t.b.e. Insektenzucht GmbH (Schnürpflingen, Germany). Culture of nematode Aphelenchoides saprophilus was obtained from Prof. Liliane Ruess, Humboldt University Berlin, Germany; nematode Bursaphelenchus mucronatus was obtained from Prof. Jiafu $\mathrm{Hu}$, Zhejiang Agriculture and Forestry Universities, China.

\subsection{Fungal strains}

Fusarium graminearum strain IFA 66 (DON chemotype) was obtained from Marc Lemmens (IFA Tull, Austria) via Thomas Miedaner (University of Hohenheim, Germany) (Miedaner et al. 2010). F. graminearum 1003 and its aurofusarin-deficient mutants generated by disruption of the polyketide synthase gene PKS12 (Malz et. al., 2005) were 
labelled $\triangle$ AUR1, $\triangle$ AUR2, and $\triangle$ AUR3. F. culmorum 3.37 was a gift from Prof. HeinzWilhelm Dehne, University of Bonn (Guo et al. 2014).

\subsection{Purification of aurofusarin}

Aurofusarin was extracted $F$. graminearum IFA 66 grown in potato medium which prepared by boiling $200 \mathrm{~g}$ potato with peel in 1 Liter tap water for $20 \mathrm{~min}$ and autoclaving the filtrate with $20 \mathrm{~g}$ glucose. The cultures were grown for two weeks at $25^{\circ} \mathrm{C}$ with shaking (200 rpm). Mycelium was harvested, freeze-dried, ground and extracted with 50 $\mathrm{ml}$ of chloroform-methanol $(80: 20, \mathrm{v} / \mathrm{v})$ per gram mycelium. The extract was cleared by centrifugation and evaporated to dryness. Aurofusarin was purified by ethanol precipitation from phenol at $50^{\circ} \mathrm{C}$ (Gray at al. 1967) and crystallization from hot glacial acetic acid (Ashley et al. 1937). Aurofusarin standard was purchased from Bioviotica Naturstoffe GmbH (Göttingen, Germany).

\subsection{Exposure of fungi to predation}

5,000 fungal spores in $5 \mu \mathrm{L}$ water were inoculated onto three rice kernels autoclaved with $200 \mu \mathrm{l}$ demineralized water (for arthropods) or onto $40 \mathrm{mg}$ rice flour autoclaved with 150 $\mu 1$ demineralized water (for nematodes) in $15 \mathrm{ml}$ Falcon tubes and incubated at $15^{\circ} \mathrm{C}$ in dark. After 7-8 days, $20 \mathrm{mg}$ (around 200 individual animal) of sprintgtail F. candida starved for two days, 2,000-3,000 individuals of nematode A. saprophilus or 1,000-2,000 individuals of nematode $B$. mucronatus in $100 \mu \mathrm{l}$ water were placed into mycelia and the incubation was continued. Controls were incubated under the same conditions without animals. Each group consisted of at least 4 replicates.

\subsection{Transcriptome analysis by RNA sequencing (RNAseq)}

F. graminearum IFA66 was exposed to F. candida as described above. After $48 \mathrm{~h}$ grazing four cultures with predators and four controls were harvested, the animals were removed and total RNA was extracted using RNAsnap method (Stead et al. 2012) modified as follows: Suspension of mycelia in of $400 \mu \mathrm{l}$ of RNA extraction solution [95\% deionized formamide, $18 \mathrm{mM}$ EDTA, $0.025 \%$ SDS, and 1\% 2-mercaptoethanol] was disrupted by shaking with zirconia beads (2.0 mm diameter, Carl Roth, Karlsruhe, Germany) in reciprocal mill MM 200 (Retsch, Haan, Germany) for 2 min at maximum power, followed by incubation at $95^{\circ} \mathrm{C}$ for $7 \mathrm{~min}$. Cells debris was removed by centrifugation at $16,000 \mathrm{x} g$ for $5 \mathrm{~min}$ at room temperature. The supernatant was transferred into RNA precipitation mixture consisting of $800 \mu \mathrm{l}$ of isobutanol, $400 \mu \mathrm{l}$ of $5 \mathrm{M}$ guanidine thiocyanate and $5 \mu \mathrm{l}$ 
of linear polyacrylamide used as co-precipitant (Co-Precipitant Pink, Bioline, London, UK). The mixture was centrifuged at $16,000 \mathrm{x}$ g for $5 \mathrm{~min}$ at room temperature and the pellet was washed with $75 \%$ ethanol, dried and dissolved in RNAase-free water.

Strand-specific cDNA libraries were prepared using Illumina's TruSeq stranded mRNA kit (75 bp paired-end) and sequenced on Illumina NextSeq 500V2. Data was analyzed using the public server of Galaxy (Afgan et al. 2016) (https://usegalaxy.org/). Before the analysis the reads were trimmed to remove low quality sequences (the least 12 bases). Reads were mapped to the reference genome ( $F$. graminearum PH-1) using Hisat2 v2.0.5.1 (Kim et al. 2005) using the default options for single end data. Cufflinks v2.2.1 (Trapnell et al. 2010) was used to determine the abundance of transcripts in FPKM (Fragments Per Kilobase of exon per Million fragments mapped), maximum intron length was set to $1000 \mathrm{nt}$ and the last annotated genome ASM24013v3 was used as reference. Cuffdiff v2.2.1.3 (Trapnell et al. 2010) was used to determine the changes in gene expression compared to the control using an FDR (false discovery rate) of 0.05. Only genes with a $\log 2$ FPKM (fold change) higher that 1.0 and a q value lower than 0.01 were considered as significantly induced.

To identify up-regulated secondary metabolite gene clusters, accession numbers of all upregulated genes were searched in the National Center for Biotechnology Information (NCBI) and in the European Bioinformatics Institute, EMBL-EBI (UniProt) databases to look for signature enzymes encoding genes for polyketide synthetases (PKS), nonribosomal peptide synthetases (NRPS) and terpenoid synthetases (TPS). Once more than $50 \%$ of the genes in the cluster and/or the signature enzyme were significantly induced ( $\log 2$ FPKM (fold change) higher than 1.0 and a q value lower than 0.01), literature related to the cluster was consulted to identify associated secondary metabolites. In this way, gene clusters for the biosynthesis of aurofusarin (Frandsen et al 2006), C62 (Sieber et al. 2014), fusarin C (Connolly et al. 2013, Niehaus et al. 2013, Sieber et al. 2014), fusaristatin A (Sieber et al. 2014, Harris et al. 2016), and terpenoids (Sieber et al. 2014) were identified. The results were corroborated with AntiSMASH 3.0 (Weber et al. 2015). Upregulated genes located immediately before and after the signature enzyme were also considered part of the cluster. Putative clusters without known signature enzymes were identified using AntiSMASH 3.0 (Weber et al. 2015) and corroborated manually. Accession numbers of the genes: fusarin C (FGSG_07798, FGSG_07800 to FGSG_07805, FGSG_13222 to FGSG_13224), cluster C62 (FGSG_10606, FGSG_10608, FGSG_10609, FGSG_10611 to FGSG_10614, FGSG_10616, FGSG_10617), aurofusarin (FGSG_02320 
to FGSG_02329), terpenoids (FGSG_01737 to FGSG_01749), fusaristatin A (FGSG_08204 to FGSG_08210, FGSG_08213, FGSG_08214), putative cluster (FGSG_10557 to FGSG_10560, FGSG_10562 to FGSG_10567, FGSG_10569 to FGSG_10571, FGSG_10573).

\subsection{Transcription of genes of aurofusarin biosynthetic pathway after predation}

After exposure to $F$. candida grazing for $0,1,2,4,8,24,48,80$ and $120 \mathrm{~h}(F$. graminearum), 80 and $120 \mathrm{~h}$ (F. culmorum) and nematode feeding for 24 and $80 \mathrm{~h}(F$. graminearum), fungal mycelia were harvested, frozen in liquid nitrogen and ground. RNA was extracted using guanidinium thiocyanate-phenol-chloroform method (Chomczynski and Sacchi, 2006) and precipitated with $4 \mathrm{M} \mathrm{LiCl}$ for 3 hours on ice and reversed transcribed with RevertAid Reverse Transcriptase (Thermo Fisher Scientific, California, USA) and random primers according to instructions of the manufacturer using $400 \mathrm{ng}$ RNA in $20 \mu \mathrm{l}$ reactions. cDNA was used as a template for PCR which contained ThermoPol Reaction Buffer (20 mM Tris-HCl, 10 mM (NH4)2SO4, $10 \mathrm{mM} \mathrm{KCl,} \mathrm{0.1 \%}$ Triton-X-100, $\mathrm{pH} 8.8$ at $25{ }^{\circ} \mathrm{C}$ ) with $2.5-4.5 \mathrm{mM} \mathrm{MgCl} 2,200 \mu \mathrm{M}$ dNTP, $0.3 \mu \mathrm{M}$ forward and reverse primers (Supplymentary File 4), SYBR Green I (Invitrogen, Karlsruhe, Germany), $1 \mathrm{mg} / \mathrm{ml}$ of bovine serum albumin, $0.03 \mathrm{U} / \mu 1$ of Taq polymerase (New England Biolabs, UK) and $1 \mu \mathrm{l}$ of cDNA as a template. PCR conditions were as follows: $95^{\circ} \mathrm{C}$ for 2 min, 35 cycles of $94^{\circ} \mathrm{C}$ for 20 to $30 \mathrm{~s}, 59^{\circ} \mathrm{C}$ for 30 to $40 \mathrm{~s}$ and $68^{\circ} \mathrm{C}$ for $30 \mathrm{~s}$, with a final extension of $68^{\circ} \mathrm{C}$ for $15 \mathrm{~min}$ followed by melting curve analysis beginning at $95^{\circ} \mathrm{C}$ with decrement of $0.5^{\circ} \mathrm{C}$ to $55^{\circ} \mathrm{C}$.

GAPDH (glyceraldehyde 3-phosphate dehydrogenase) and EFIA (elongation factor 1alpha) were used as reference genes (Extended data 6). Amplification efficiency for each gene was calculated determined with the help of serial dilutions (Pfaffl 2001). Four biological replicates were analyzed.

\subsection{Food preference of fungivorous arthropods}

Springtails ( $F$. candida) were cultivated on Petri dishes (92 $\mathrm{mm}$ diameter) filled with a mixture of solidified plaster of Paris and activated charcoal (9:1). The cultures were maintained in dark at $15^{\circ} \mathrm{C}$ and fed with baker's yeast. Fungal cultures were grown on PDA medium (made the same as 2.3. Purification of aurofusarin) at $25^{\circ} \mathrm{C}$ for $7-8$ days (until red pigment in wide-type strain could be seen) in dark. Agar plagues were cut from the edge of fungal colonies using a sterile cord borer (12 $\mathrm{mm}$ in diameter) and placed on 
opposite sides of Petri dishes filled with plaster of Paris - activated charcoal mixture. Twenty $F$. candida individuals starved for two days were placed into the center of the Petri dish and the plates were incubated at $15^{\circ} \mathrm{C}$ in dark. The number of collembolans on each mycelium was counted at several time points. The same conditions were used for isopod $T$. tomentosa but eight animals were used.

Feeding experiments with $T$. molitor were carried out with fungal mycelia as well as with wheat flour amended with aurofusarin. In the first experiment $F$. graminearum strains were cultured on glass slides covered with PDA as above. After $7-8$ days at $25^{\circ} \mathrm{C}$, the slides were placed on the opposite sides of Petri dishes $150 \times 20 \mathrm{~mm}$ and eight T. molitor larvae were placed in the middle of the plates. The number of animals visiting each fungal culture was recorded by taking photos at time intervals for $12 \mathrm{~h}$ and counting animals inside rectangles $13 \times 6 \mathrm{~cm}$ drawn around each slide. At least 4 replicates were used for each treatment and each experiment was repeated at least twice. In the second experiment wheat flour was amended with aurofusarin to a concentration of $10,000 \mu \mathrm{g} / \mathrm{g}$. Portions of $0.4 \mathrm{~g}$ of wheat flour with aurofusarin and pure wheat flour were placed put on opposite sides of three Petri dishes. Five larvae of T. molitor were added to each Petri dish and the cultures were kept for 4 days at room temperature. Then the animals were removed and the weight of remaining wheat flour with and without aurofusarin, mixed with fecal pellets left by the animals, was determined.

\subsection{Effect of aurofusarin on the fitness of fungivorous arthropods}

\subsubsection{Springtails and white isopods}

Ten $F$. candida or five $T$. tomentosa individuals were placed into a Petri dish filled with a layer of Plaster of Paris. 7-8 days old mycelium of F. graminearum 1003 or aurofusarindeficient mutants were taken from PDA medium using a cork borer of $12 \mathrm{~mm}$ diameter and placed in the center of the Petri dish with animals. New mycelium was provided every 10 days (for F. candida) and every week (for T. tomentosa). Tap water was added when necessary to keep humidity. F. candida was maintained at $18^{\circ} \mathrm{C}$ and $\mathrm{T}$. tomentosa at $25^{\circ} \mathrm{C}$. At the beginning of the experiment and after 5 weeks, all animals were transferred to a Petri dish containing a layer of plaster with charcoal for size measurement. A digital photograph was taken and the length and width of each animal were determined using Image J (Maryland, USA). Each treatment consisted of at least four replicates.

\subsubsection{Mealworms}


Wheat flour were amended with aurofusarin dissolved in chloroform and the solvent was evaporated, leading to aurofusarin concentrations of $0,1,10,100,1,000$ and 10,000 $\mu \mathrm{g} / \mathrm{g}$. Ten larvae of $T$. molitor were weighed individually and placed on $1 \mathrm{~g}$ of wheat flour amended with aurofusarin. After 10 days at $18^{\circ} \mathrm{C}$ in dark, the weight of the animals was determined again. Each treatment consisted of four replicates; two independent experiments were carried out.

\subsection{Toxicity of aurofusarin to insect cells in tissue culture}

Spodoptera frugiperda 9 (Sf9) cell line was maintained on Sf-900 II medium (Thermo Fisher Scientific China, Shanghai, China) and grown at $28^{\circ} \mathrm{C}$. The cells were seeded into 96-well cell culture plates at a concentration of $8 \times 103$ cells per well and treated with aurofusarin $[0,1.25,2.5,5,10,20,40,80,100,200,400,600,800$ and $1000 \mu \mathrm{M}$ dissolved in dimethyl sulfoxide (DMSO) for 24 h. MTT (3-(4,5-dimethylthiazol-2-yl)-2,5diphenyltetrazolium bromide) solution $(0.5 \mathrm{mg} / \mathrm{mL})$ was added to each well and incubation was continued for $4 \mathrm{~h}$. Formazan precipitate was dissolved in $150 \mu \mathrm{L}$ of dimethyl sulfoxide (DMSO) and the absorbance of the solution at $490 \mathrm{~nm}$ was determined. The test was performed in triplicate.

\subsection{Determination of aurofusarin and viomellein by HPLC}

$F$. graminearum and $F$. culmorum cultures were treated with springtail $F$. candida and mealworm T. molitor as described in section 2.4. The animals were removed and remaining material was freeze-dried and weighed. Aurofusarin was extracted into $2 \mathrm{ml}$ of chloroform/methanol (80:20) overnight. After centrifugation at 14,000 rpm for $20 \mathrm{~min}$, $600 \mu \mathrm{l}$ of the supernatant were dried and the residue was dissolved in $80 \mu 1$ DMSO. Aurofusarin content was determined by HPLC with a diode-array detector (Varian Prostar), using polar modified RP-18-column (Polaris 3 C18-Ether, 100 x 2.0 mm; Varian, Darmstadt, Germany) kept at $40^{\circ} \mathrm{C}$. Gradient elution with solvent A (water with $0.05 \%$ acetic acid and 5\% acetonitrile) and B (methanol with $0.05 \%$ acetic acid) was used as follows: $0.0-0.1 \mathrm{~min} 60 \% \mathrm{~B}, 0.1-12 \mathrm{~min}$ increase to $98 \% \mathrm{~B}, 12-14 \mathrm{~min} 98 \% \mathrm{~B}, 14-15 \mathrm{~min}$ decrease to $60 \% \mathrm{~B}, 15-25 \mathrm{~min} 60 \% \mathrm{~B}$. Light absorption was monitored at $243 \mathrm{~nm}$ for aurofusarin determination detection and at $270 \mathrm{~nm}$ for viomellein analysis.

\subsection{Statistics and reproducibility}

Bar graphs show means $\pm \mathrm{SE}$; line graph shows means $\pm 95 \% \mathrm{CI}$; box plots show medians and lower and quartiles (Q1 and Q3) with whiskers showing the largest (smallest) 
observation or 1.5-fold of the interquartile range, whichever is smaller (larger) [Q1$1.5 *(\mathrm{Q} 3-\mathrm{Q} 1), \mathrm{Q} 3+1.5 *(\mathrm{Q} 3-\mathrm{Q} 1)]$. Significance of difference between means was tested by un-paired t-test. The number of biological replicates is listed in figure captures and the reproducibility of the data is all listed in Extended data 7.

\section{References}

Afgan, E., Baker, D., van den Beek, M., Blankenberg, D., Bouvier, D., Cech, M., Chilton, J., Clements, D., Coraor, N., Eberhard, C., Grüning, B., Guerler, A., HillmanJackson, J., Von Kuster, G., Rasche, E., Soranzo, N., Turaga, N., Taylor, J., Nekrutenko, A. and Goecks, J. (2016). The galaxy platform for accessible, reproducible and collaborative biomedical analyses: 2016. Nucleic Acids Research 8: 3-10.

Ashley, J.N., Hobbs, B.C. and Raistrick, H. (1937). Studies in the biochemistry of microorganisms: The crystalline colouring matters of Fusarium culmorum (W. G. Smith) Sacc. and related forms. Biochemical Journal 31: 385-397.

A'Bear, A.D., Jones, T.H. and Boddy, L. (2014). Size matters: What have we learnt from microcosm studies of decomposer fungus-invertebrate interactions? Soil Biology and Biochemistry 78: 274-283.

Bleuler-Martínez, S., Butschi, A., Garbani, M., Wälti, M.A., Wohlschlager, T., Potthoff, E., Sabotic, J., Pohleven, J., Lüthy, P., Hengartner, M.O., Aebi, M. and Künzler, M. (2011). A lectin-mediated resistance of higher fungi against predators and parasites. Molecular Ecology 20: 3056-3070.

Boddy, L., Wood, J., Redman, E., Hynes, J. and Fricker, M.D. (2010). Fungal network responses to grazing. Fungal Genetics and Biology 47: 522-530.

Brandt, P., García-Altares, M., Nett, M., Hertweck, C. \& Hoffmeister, D. (2017) Induced chemical defense of a mushroom by a double-bond-shifting polyene synthase. Angewandte Chemie, International Edition 56, 5937-941.

Brimble, M.A., Duncalf, L.J. \& Nairn, M.R. Pyranonaphthoquinone antibiotics-isolation, structure and biological activity. Natural Products Reports. 16, 267-281 (1999). 
Calvo, A. M. \& Cary, J. W. Association of fungal secondary metabolism and sclerotial biology. Frontiers in Microbiology 6, 62 (2015).

Cary, J.W., Harris-Coward, P.Y., Ehrlich, K.C., Di Mavungu, J.D., Malysheva, S.V., De Saeger, S., Dowd, P.F., Shantappa, S., Martens, S.L. and Calvo, A.M. (2014). Functional characterization of a veA-dependent polyketide synthase gene in Aspergillus flavus necessary for the synthesis of asparasone, a sclerotium-specific pigment. Fungal Genetics and Biology 64: 25-35.

Chomczynski, P. \& Sacchi, N. (2006). The single-step method of RNA isolation by acid guanidinium thiocyanate-phenol-chloroform extraction: twenty-something years on. Nature Protocols 1: 581-585.

Choque, E., Rayess, Y.E. \& Raynal, J. Fungal naphtho- $\gamma$-pyrones-secondary metabolites of industrial interest. Applied Microbiology and Biotechnology 99, 1081-96 (2015).

Connolly, L.R., Smith, K.M. \& Freitag, M. (2013). The Fusarium graminearum histone H3 K27 methyltransferase KMT6 regulates development and expression of secondary metabolite gene clusters. Plos Genetics 9: e1003916 (2013).

Crowther, T.W., Boddy, L. and Hefin Jones, T. (2012). Functional and ecological consequences of saprotrophic fungus-grazer interactions. The ISME Journal 6: 1992-2001.

Crump, C.E., Rollins, B.S. \& Hayden, F.G. (1990). In vitro antiviral activity and cytotoxicity of aspirin: lack of selective activity against influenza a virus or rhinovirus. Antiviral Chemistry and Chemotherapy. 1: 217-221.

Donner, C.D. (2015) Naphthopyranones-isolation, bioactivity, biosynthesis and synthesis. Natural Products Reports. 32, 578-604 (2015).

Dowd, P.F. (1992). "Insect interaction with mycotoxin-producing fungi and their hosts. " Handbook of Applied Mycology. pp 137-155.

Dowd, P.F., Miller, J.D. and Greenhalgh, R. (1989). Toxicity and interactions of some Fusarium graminearum metabolites to caterpillars. Mycologia. 81, 646-650.

Dvorska, J.E., Surai, P.F., Speake, B.K. and Sparks, N.H. (2001). Effect of the mycotoxin aurofusarin on the antioxidant composition and fatty acid profile of quail eggs. British Poultry Science 42: 643-649. 
Frandsen, R.J, Nielsen, N.J., Maolanon, N., Sorensen, J.C., Olsson, S., Nielsen, J. and Giese, H. (2006). The biosynthetic pathway for aurofusarin in Fusarium graminearum reveals a close link between the naphthoquinones and naphthopyrones. Molecular Microbiology 61: 1069-1080.

Gray, J.S., Martin, G.C.J. and Rigby, W. (1976). Aurofusarin. Journal of the Chemical Society C: Organic Chemistry: 2580-2587.

Guo, Z.Q., Döll, K., Dastjerdi, R., Karlovsky, P., Dehne, H.W. and Altincicek, B. (2014). Effect of fungal colonization of wheat grains with Fusarium spp. on food choice, weight gain and mortality of meal beetle larvae (Tenebrio molitor). Plos One 9: e100112.

Harris, L.J., Balcerzak, M., Johnston, A., Schneiderman, D. \& Ouellet, T. (2016). Hostpreferential Fusarium graminearum gene expression during infection of wheat, barley, and maize. Fungal Biology 120, 111-123.

Hillmann, F., Novohradska, S., Mattern, D.J., Forberger, T., Heinekamp, T., Westermann, M., Winckler, T. and Brakhage, A.A. (2015). Virulence determinants of the human pathogenic fungus Aspergillus fumigatus protect against soil amoeba predation. Environmental Microbiology 17: 2858-2869.

Kim, D., Langmead, B. and Salzberg, S.L. (2015). HISAT: a fast spliced aligner with low memory requirements. Nature Methods 12: 357-360.

Künzler, M. (2015). Hitting the sweet spot-glycans as targets of fungal defense effector proteins. Molecules 20: 8144-8167.

Malz, S., Grell, M.N., Thrane, C., Maier, F.J., Rosager, P., Felk, A., Albertsen, K.S., Salomon, S., Bohn, L., Schäfer, W. and Giese, H. (2005). Identification of a gene cluster responsible for the biosynthesis of aurofusarin in the Fusarium graminearum species complex. Fungal Genetics and Biology 42, 420-433.

Mapari, S.A., Meyer, A.S., Thrane, U. and Frisvad, J.C. (2009). Identification of potentially safe promising fungal cell factories for the production of polyketide natural food colorants using chemotaxonomic rationale. Microbial Cell Factories 8: 24. 
McGonigle, M.C. (2007). Effects of animals grazing on fungi. In: The Mycota IV: Environmental and Microbial Relationships (C P. Kubicek, I. S. Druzhinina, eds.) Springer, Berlin, Heidelberg, pp 201-212.

Medentsev, A.G. \& Akimenko, V.K. (1998). Naphthoquinone metabolites of the fungi. Phytochemistry. 47, 935-959.

Miedaner, T., Bolduan, C. and Melchinger. (2010). Aggressiveness and mycotoxin production of eight isolates each of Fusarium graminearum and Fusarium verticillioides for ear rot on susceptible and resistant early maize inbred lines. European Journal of Plant Pathology 127: 113-123.

Niehaus, E.M., Kleigrewe, K., Wiemann, P., Studt, L., Sieber, C.M., Connolly L.R., Freitag, M., Güldener, U., Tudzynski, B. and Humpf, H.U. (2013). Genetic manipulation of the Fusarium fujikuroi fusarin gene cluster yields insight into the complex regulation and fusarin biosynthetic pathway. Chemistry \& Biology 20: $1055-1066$.

Pfaffl, M.W. (2001). A new mathematical model for relative quantification in real-time RT-PCR. Nucleic Acids Research. 29: e45 .

Plaza, D.F., Schmieder, S.S., Lipzen, A., Lindquist, E. and Künzler, M. (2015). Identification of a novel nematotoxic protein by challenging the model mushroom Coprinopsis cinerea with a fungivorous nematode. G3: Genes, Genomes, Genetics 6: 87-98.

Põldmaa, K. (2011). Tropical species of Cladobotryum and Hypomyces producing red pigments. Studies in Mycology 68: 1-34.

Rohlfs, M. (2015). Fungal secondary metabolite dynamics in fungus-grazer interactions: novel insights and unanswered questions. Frontiers in Microbiology 5, 788.

Rohlfs, M. and Churchill, A.C. (2011). Fungal secondary metabolites as modulators of interactions with insects and other arthropods. Fungal Genetics and Biology 48: 23-34.

Ruess L, Lussenhop J. (2005). Trophic interactions of fungi and animals. In: The Fungal Community, $3^{\text {rd }}$ Ed. (J. Dighton J, J. F. White, P. Oudemans, eds.) CRC Press: Boca Raton, pp. 581-598. 
Sabotič, J., Ohm, R.A. \& Künzler, M. (2016). Entomotoxic and nematotoxic lectins and protease inhibitors from fungal fruiting bodies. Applied Microbiology and Biotechnology 100: 91-111.

Schubert, M., Bleulaer-Martinez, S., Butschi, A., Wälti, M.A., Egloff, P., Stutz, K., Yan, S., Wilson, I.B.H., Hengartner, M.O., Aebi, M., Allain, F.H.T. and Künzler, M. (2012). Plasticity of the $\beta$-trefoil protein fold in the recognition and control of invertebrate predators and parasites by a fungal defence system. Plos Pathogens 8: e1002706.

Scott, J. G. (1999). Cytochromes P450 and insecticide resistance. Insect Biochemistry and Molecular Biology 29: 757-777.

Schwenk, D., Nett, M., Dahse, H.M., Horn, U., Blanchette, R.A. and Hoffmeister, D. (2014). Injury-induced biosynthesis of methyl-branched polyene pigments in a white-rotting basidiomycete. Journal of Natural Products 77: 2658-2663.

Sieber, C.M., Lee, W., Wong, P., Münsterkötter, M., Mewes, H.W., Schmeitzl, C., Varga, E., Berthiller, F., Adam, G. and Güldener, U. (2014). The Fusarium graminearum genome reveals more secondary metabolite gene clusters and hints of horizontal gene transfer. Plos One 9, e110311.

Sørensen, J.L., Nielsen, K.F. and Sondergaard, T.E. (2012). Redirection of pigment biosynthesis to isocoumarins in Fusarium. Fungal Genetics and Biology 49: 613618.

Staaden, S., Milcu, A., Rohlfs, M. and Scheu, S. (2010). Fungal toxins affect the fitness and stable isotope fractionation of Collembola. Soil Biology and Biochemistry 42: $1766-1773$.

Stead, M.B., Agrawal, A., Bowden, K.E., Nasir, R., Mohanty, B.K., Meagher, R.B. and Kushner, S.R. (2012). RNAsnap ${ }^{\mathrm{TM}}$ : a rapid, quantitative and in expensive, method for isolating total RNA from bacteria. Nucleic Acids Research. 20: e156-e165.

Stötefeld, L., Scheu, S. \& Rohlfs, M. (2012). Fungal chemical defence alters densitydependent foraging behaviour and success in a fungivorous soil arthropod. Ecological Entomology 37: 323-329.

Trapnell, C., Williams, B.A., Pertea, G., Mortazavi, A., Kwan, G., van Baren, M.J., Salzberg, S.L., Wold, B.J. and Pachter, L. (2010). Transcript assembly and 
quantification by RNA-Seq reveals unannotated transcripts and isoform switching during cell differentiation. Nature Biotechnology 28: 511-515.

Trienens M., Keller N.P. and Rohlfs M. (2010). Fruit, flies and filamentous fungi experimental analysis of animal-microbe competition using Drosophila melanogaster and Aspergillus mould as a model system. Oikos 119: 1765-1775.

Trienens, M. and Rohlfs, M. (2012). Insect-fungus interference competition - The potential role of global secondary metabolite regulation, pathway-specific mycotoxin expression and formation of oxylipins. Fungal Ecology 5: 191-199.

Wicklow, D.T. (1988). "Metabolites in the coevolution of fungal chemical defence systems. In: Coevolution of Fungi with Plants and Animals (K. Pyrozynski, ed.) Academic Press, London. pp 173-201.

Weber, T., Blin, K., Duddela, S., Krug, D., Kim, H.U., Bruccoleri, R., Lee, S.Y., Fischbach, M.A., Müller, R., Wohleben, W., Breitling, R., Takano, E. and Medema, H.M. (2015). antiSMASH 3.0-a comprehensive resource for the genome mining of biosynthetic gene clusters. Nucleic Acids Research 43, W237W243.

Wohlschlager, T., Butschi, A., Grassi, P., Sutov, G., Gauss, R., Hauck, D., Schmieder, S.S., Knobel, M., Titz, A., Dell, A., Haslam, S.M., Hengartner, M.O., Aebi, M. and Künzler, M. (2014). Methylated glycans as conserved targets of animal and fungal innate defense. Proceedings of the National Acadeny of Sciences of the United States of America. 111: 2787-2796.

Yin, W.B., Amaike S., Wohlbach, D.J., Gasch, A.P., Chiang, Y.M., Wanf, C.C., Bok, J.W., Rohls, M., Keller, N.P. (2012). An Aspergillus nidulans bZIP response pathway hardwired for defensive secondary metabolism operates through aflR. Molecular Microbiology 83, 1024-1034. 


\section{Extended data}

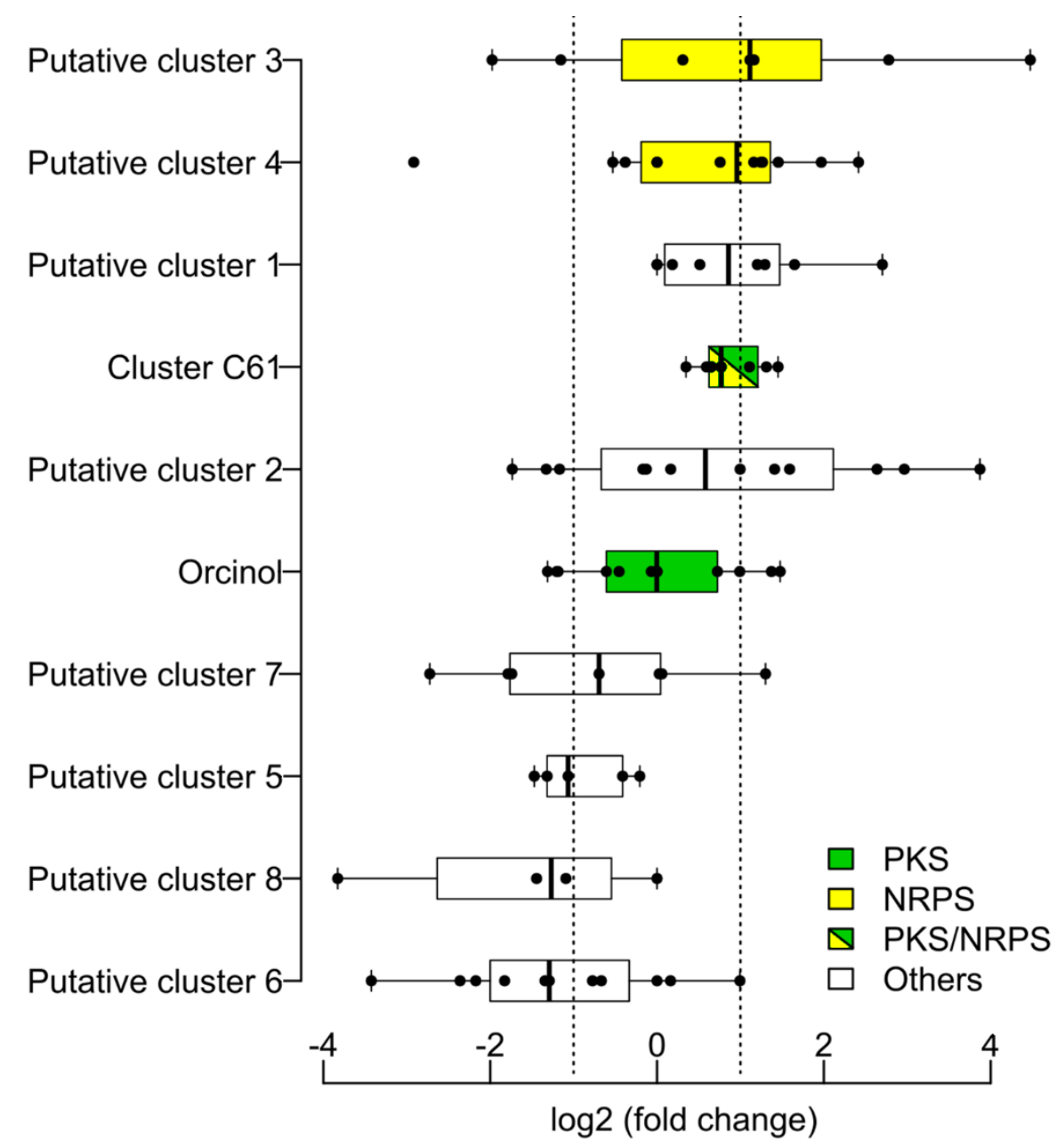

Extended Data Fig. 1 | Further gene clusters for secondary metabolites in Fusarium graminearum up-regulated by springtail grazing. $F$. graminearum was exposed to springtail F. candida for 48 hours and mRNA levels were determined by RNAseq. Up-regulated clusters were identified as having $>50 \%$ of the genes and/or the gene encoding the signatures enzyme induced [ $\log 2$ FPKM (fold change) higher than 1.0 (dotted line) and a q value lower than 0.01]. Accession numbers: fusarin C (FGSG_07798, FGSG_07800 to FGSG_07805, FGSG_13222 to FGSG_13224), cluster C62 (FGSG_10606, FGSG_10608, FGSG_10609, FGSG_10611 to FGSG_10614,FGSG_10616, FGSG_10617), aurofusarin (FGSG_02320 to FGSG_02329), terpenoids (FGSG_01737 to FGSG_01749), fusaristatin A (FGSG_08204 to FGSG_08210, FGSG_08213, FGSG_08214), putative cluster(FGSG_10557 to FGSG_10560, FGSG_10562 to FGSG_10567, FGSG_10569 to FGSG_10571, FGSG_10573). RNAseq analysis was carried out on four biological replicates. Box plots show the median for each cluster and lower and upper quartiles (Q1 and Q3). Whiskers show the largest (smallest) observation or 1.5-fold of the interquartile range, whichever is smaller (larger) $\left[\mathrm{Q} 1-1.5^{*}(\mathrm{Q} 3-\mathrm{Q} 1)\right.$ and $\left.\mathrm{Q} 3+1.5^{*}(\mathrm{Q} 3-\mathrm{Q} 1)\right]$. 

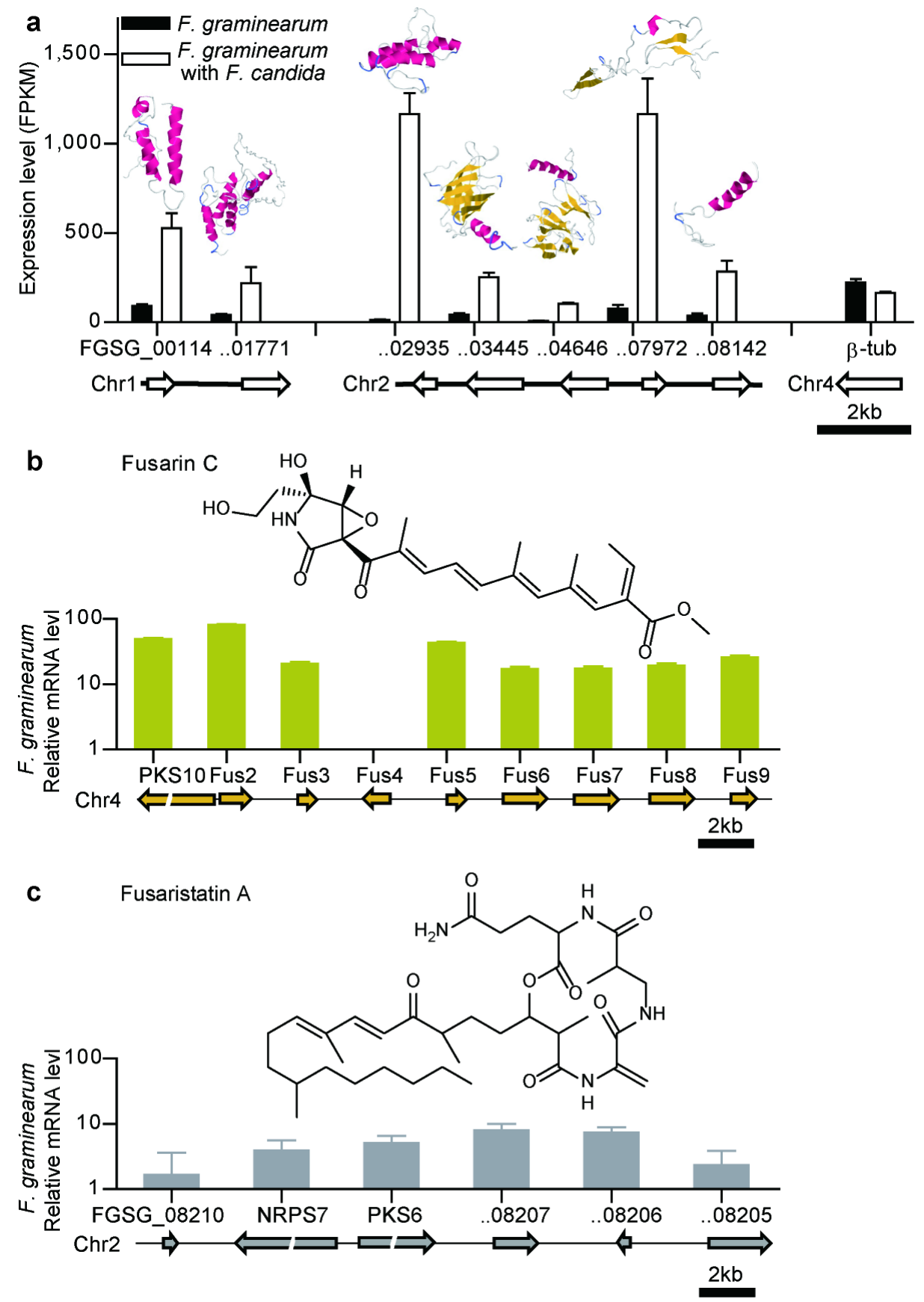

Extended Data Fig. 2 | Springtails grazing induces cysteine-rich proteins-codifying-genes, fusarin $\mathrm{C}$ and fusaristatin A biosynthetic pathways in $\boldsymbol{F}$. graminearum. a, Fragments per kilobase of mapped reads (FPKM) generated by Cufflinks of 7 most up-regulated small secreted cysteine-rich proteins-codifying-genes in $F$. graminearum when feeding by springtail $F$. candida for 48 hours according to RNAseq data and their protein structure, $\beta$-tubulin gene is presented as reference. b,c, Up-regulation of fusarin $\mathrm{C}$ and fusaristatin A biosynthetic pathways in $F$. graminearum after exposure to springtail $F$. candida for $48 \mathrm{~h}$. RNAseq analysis was carried out with four biological replicates; the whiskers show standard errors. 


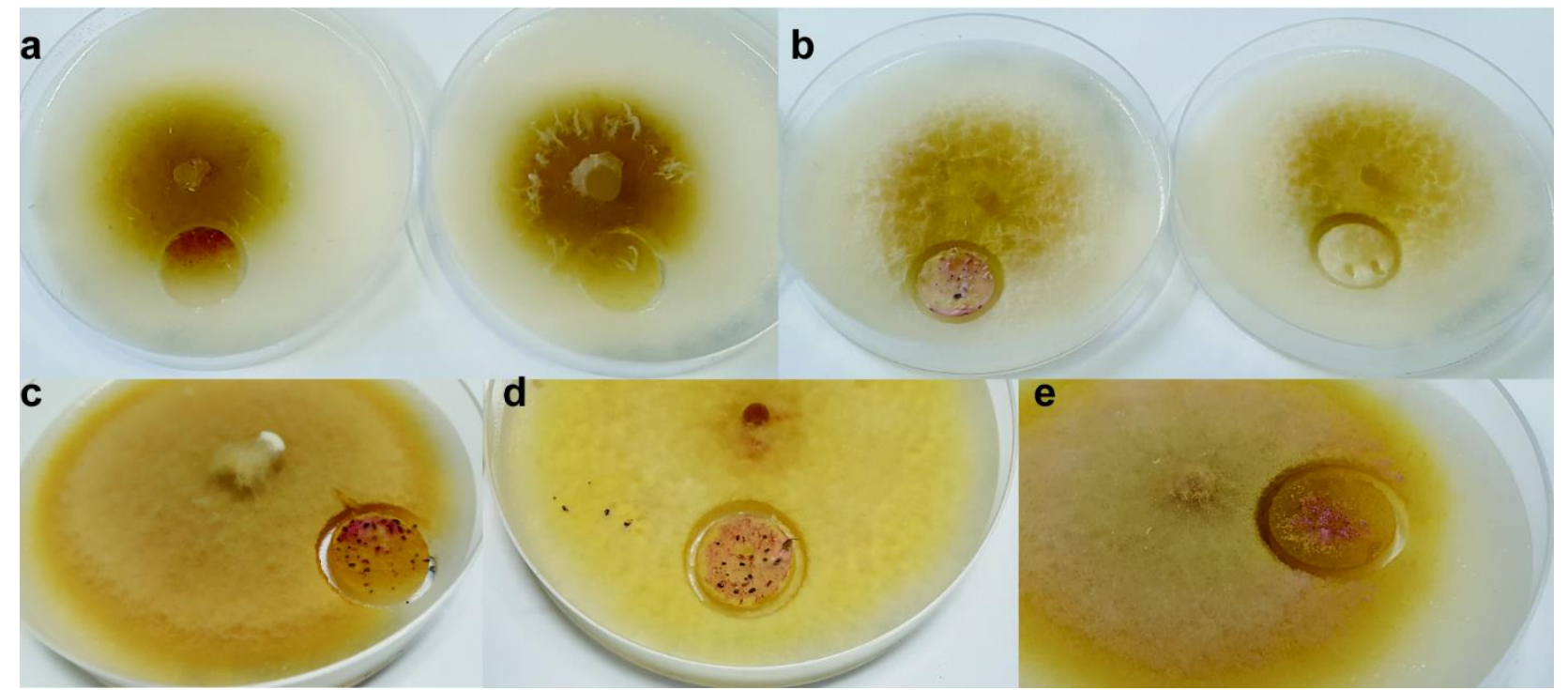

Extended Data Fig. 3 | Stimulation of the synthesis red pigment in Fusarium spp. by grazing of predators. a, Left: F. poae DSMZ 62376 culture on rice agar with arena exposure to $F$. candida grazing for 2 days; right: control. b, $F$. venenatum RD15 culture with arena exposure to $F$. candida grazing for 2 days; right: control. c, F. sporotrichoides IPP 0249 culture with arena exposed to isopod Porcellionides pruinosus for 2 days. d, F. venenatum RD 90 culture with arena exposure to isopod Porcellionides pruinosus for 2 days. e, F. avenaceum BBA 92013 culture with arena exposure to $F$. candida for 2 days. The animals were confined to arenas of $15 \mathrm{~mm}$ diameter with the help of plastic cylinders and removed immediately before the photos were taken. 


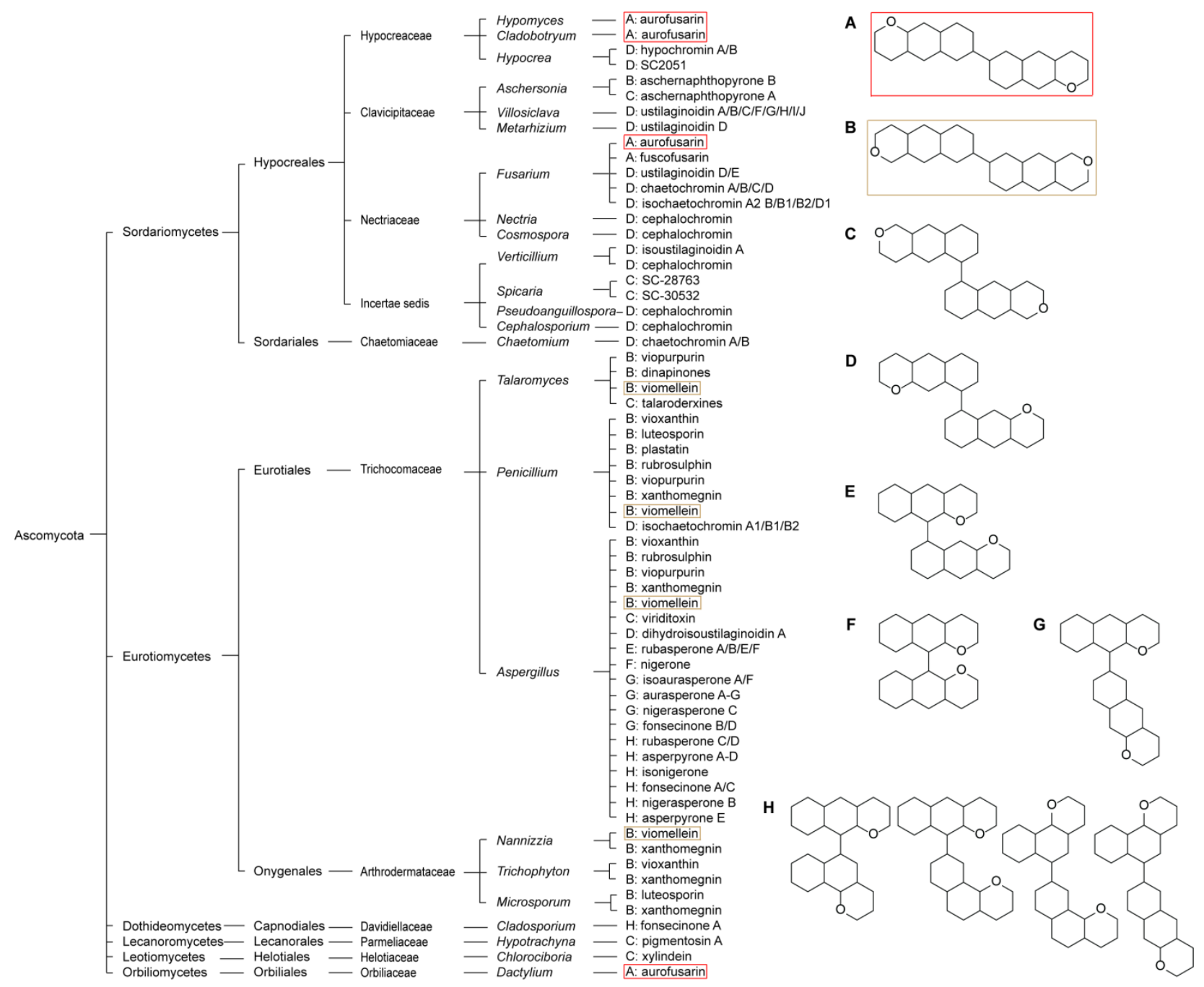

Extended Data Fig. 4 | Production of dimeric napththopyrones by ascomycetous fungi. Taxonomic affiliation of selected fungal genera that produce dimeric napththopyrones is shown on the left. Schematic structures of fungal bis-naphthopyrones are shown on the right. Structure classes A to I contain dimeric naphtho- $\alpha$-pyrones and naphtho- $\gamma$-pyrones consisting of linear heptaketides; the classes differ by the location of links between monomers and by the presence of $\alpha$-pyrone or $\gamma$ pyrone. Metabolites of class J consist of a linear and an angular heptaketides; all metabolites of class $\mathrm{J}$ listed here contain a $\gamma$-pyrone moiety. 


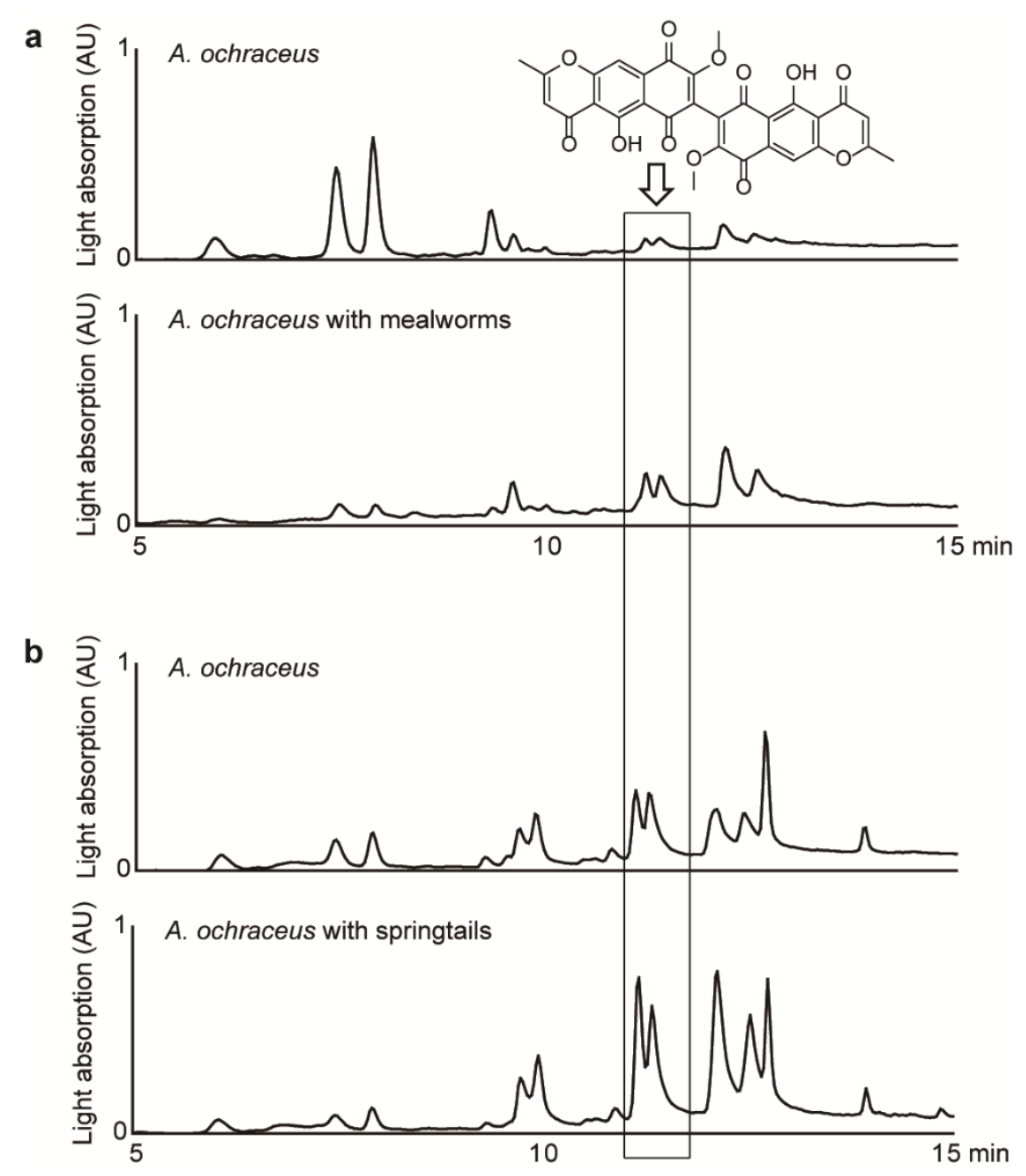

Extended Data Fig. 5 | Invertebrate grazing stimulates viomellein production by Aspergillus

ochraceus. a, Aspergillus ochraceus was grown on maize flour medium for 5 days. Eight larvae of T. molitor were added to the cultures and kept on fungal cultures for 6 days. Cultures without animals served as controls. $\mathrm{n}=5 \mathbf{b}$, A. ochraceus 6692 were grow on rice medium for 5 days. $20 \mathrm{mg}$ of $F$. candida starved for two days were added and allowed to feed on the culture for 6 days. $n=6$ Extracts of the cultures into chloroform-methanol (80:20) were analysed by HPLC with a light absorption detector set to $270 \mathrm{~nm}$. Viomellein appears as two peaks because it consists of two diastereomers (atropisomers) due to restricted rotation around the bond connecting the monomers. 
Extended Data Fig. 6 | Primers were used for qRT-PCR analysis.

\begin{tabular}{|c|c|c|c|c|}
\hline Target sequence & $5^{\prime}$ to $3^{\prime}$ sequence & $\begin{array}{c}\text { Amplicon } \\
\text { size }\end{array}$ & Application & $\begin{array}{l}\text { Related } \\
\text { reference }\end{array}$ \\
\hline GAPDH F & CTACATGCTCAAGTACGACTCTTCC & & & Hallen-adams et \\
\hline GAPDH R & GCCGGTCTCGGACCACTTG & & परा-क & al. 2011 \\
\hline EF1A F & AAAGACCCTCCTTGAGGCCATTGA & & $a \mathrm{RT}$ TPCR & Hallen-adams et \\
\hline EF1A R & ACTTCAGTGGTGACGTTGGAAGGA & & परा-1 & al. 2011 \\
\hline AurR1 F & TACAGATGACGCACTGCTGA & \multirow{2}{*}{196} & \multirow{2}{*}{ qRT-PCR } & FGSG_02320/ \\
\hline AurR1 R & CGACAACTGTGCCAAGTCAA & & & Fcul_02391 \\
\hline PKS12 F & CAGACTACGCCCAATGACTAC & \multirow{2}{*}{186} & \multirow{2}{*}{ qRT-PCR } & FGSG_02324/ \\
\hline PKS12 R & GTCTCCATAAACACCAACCACA & & & Fcul_02395 \\
\hline Gip1 F & CCGGAGTTGGAGAGTGGA & \multirow{2}{*}{145} & \multirow{2}{*}{ qRT-PCR } & FGSG_02328/ \\
\hline Gip2 R & TCAAACCACGGATCAAAGTCT & & & Fcul_02399 \\
\hline AurJ F & CTGTGTCCTCAGCAGCTAAG & \multirow{2}{*}{201} & \multirow{2}{*}{ qRT-PCR } & \multirow{2}{*}{ FGSG_02326 } \\
\hline AurJ R & TGTTGGGTAGAAGCTCGCT & & & \\
\hline AurJ F* & ACAGTTTCAGGGCTGCTTCAG & \multirow{2}{*}{170} & \multirow{2}{*}{ qRT-PCR } & \multirow{2}{*}{ Fcul_02392 } \\
\hline AurJ R* & TGCGAATGCTGTGTCGTCAG & & & \\
\hline AurO F & GACTCTGTCACCATCGACAT & \multirow{2}{*}{223} & \multirow{2}{*}{ qRT-PCR } & FGSG_02321/ \\
\hline AurO R & CTACGCCTAGACCGACATAGT & & & Fcul_02392 \\
\hline
\end{tabular}


Extended Data Fig. 7 | Details of statistical test in Figure 4, statistical was done by Graphpad Prism 5.

\begin{tabular}{|c|c|c|c|}
\hline Figure & Sample size & Statistical test & Values \\
\hline 4a length $\mathrm{Fg} / \mathrm{Fg} \Delta \mathrm{AUR} 1$ & $\mathrm{n}=4$, each contains at least 10 animals at beginning & unpaired two-tailed t-test & $\mathrm{P}<0.0001$ \\
\hline 4a length $\mathrm{Fg} / \mathrm{Fg} \Delta \mathrm{AUR} 2$ & as above & as above & $\mathrm{P}<0.0001$ \\
\hline 4a length $\mathrm{Fg} / \mathrm{Fg} \Delta \mathrm{AUR} 3$ & as above & unpaired one-tailed t-test & $\mathrm{P}=0.01$ \\
\hline 4a width $\mathrm{Fg} / \mathrm{Fg} \Delta \mathrm{AUR} 1$ & as above & unpaired two-tailed t-test & $\mathrm{P}=0.0001$ \\
\hline 4a width $\mathrm{Fg} / \mathrm{Fg} \Delta \mathrm{AUR} 2$ & as above & as above & $\mathrm{P}<0.0001$ \\
\hline 4a width $\mathrm{Fg} / \mathrm{Fg} \Delta \mathrm{AUR} 3$ & as above & as above & $\mathrm{P}=0.0085$ \\
\hline 4b length $\mathrm{Fg} / \mathrm{Fg} \Delta \mathrm{AUR} 1$ & $\mathrm{n}=4$, each contains 5 animals at beginning & as above & $\mathrm{P}<0.0001$ \\
\hline 4b length $\mathrm{Fg} / \mathrm{Fg} \Delta \mathrm{AUR} 2$ & as above & as above & $\mathrm{P}<0.0001$ \\
\hline 4b length $\mathrm{Fg} / \mathrm{Fg} \Delta \mathrm{AUR} 3$ & as above & as above & $\mathrm{P}<0.0001$ \\
\hline 4b width $\mathrm{Fg} / \mathrm{Fg} \Delta \mathrm{AUR} 1$ & as above & as above & $\mathrm{P}=0.0481$ \\
\hline 4b width $\mathrm{Fg} / \mathrm{Fg} \Delta \mathrm{AUR} 2$ & as above & as above & $\mathrm{P}=0.0029$ \\
\hline $4 \mathrm{~b}$ width $\mathrm{Fg} / \mathrm{Fg} \Delta \mathrm{AUR} 3$ & as above & as above & $\mathrm{P}=0.0001$ \\
\hline $4 \mathrm{c} 0 / 0.001 \mathrm{mg} / \mathrm{g}$ aurofusarin containing wheat & $\mathrm{n}=4$, each contains at least 10 animals at beginning & as above & $\mathrm{P}=0.6282$ \\
\hline $4 \mathrm{c} 0 / 0.01 \mathrm{mg} / \mathrm{g}$ aurofusarin containing wheat & as above & as above & $\mathrm{P}=0.2906$ \\
\hline $4 \mathrm{c} 0 / 0.1 \mathrm{mg} / \mathrm{g}$ aurofusarin containing wheat & as above & as above & $\mathrm{P}=0.6810$ \\
\hline $4 \mathrm{c} 0 / 1 \mathrm{mg} / \mathrm{g}$ aurofusarin containing wheat & as above & as above & $\mathrm{P}=0.2231$ \\
\hline $4 \mathrm{c} 0 / 10 \mathrm{mg} / \mathrm{g}$ aurofusarin containing wheat & as above & as above & $\mathrm{P}=0.0027$ \\
\hline $4 \mathrm{e} 0 / 10 \mathrm{mg} / \mathrm{g}$ aurofusarin containing wheat & $\mathrm{n}=3$, each with 5 mealworms consuming & as above & $\mathrm{P}=0.0033$ \\
\hline
\end{tabular}



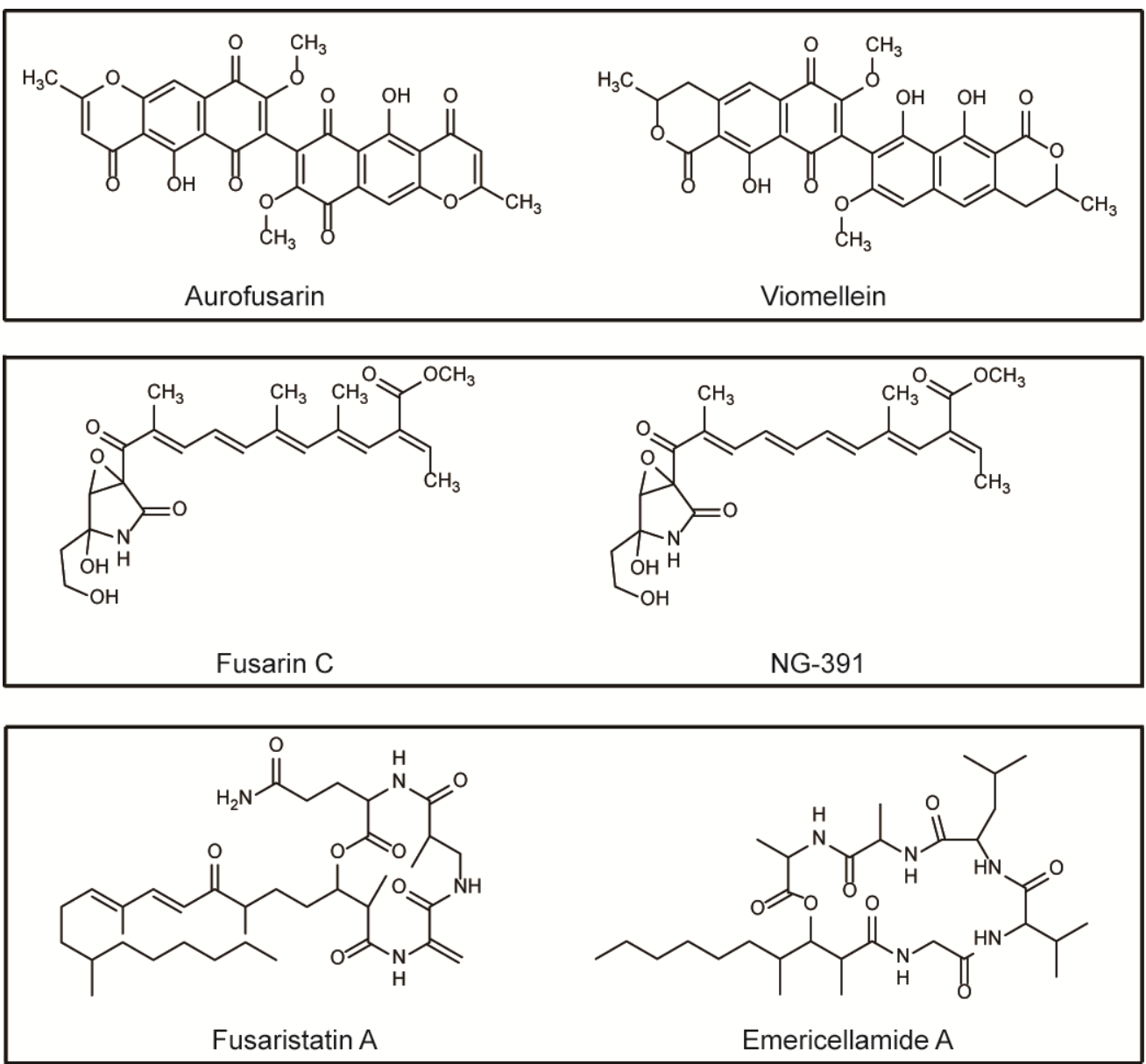

Extended Data Fig. 8 | Induced secondary metabolites (auorufsarin, viomellein and fusaristatin A) in $F$. graminearum when predated by $F$. candida, and their similar compounds. 


\section{Chapter 5: General discussion}

Small animals interact with various fungal species in soil ecosystems. In our study, we focused on mycotoxin-producing fungi of the genera Fusarium, Aspergillus and Penicillium. Some species of these genera cause diseases in plants and animals, contaminate crop products, lead to reduction of crop yield and economic loss. They are well-known to produce diverse toxic secondary metabolites, such as deoxynivalenol, zearalenone, nivalenol, fumonisin, ochratoxin A, citrinin, and penicillic acid (Karlovsky, 1999). Interactions between mycotoxin-producing fungi and invertebrates have rarely been studied.

Our study revealed that grazing of the collembolan Folsomia candida on F. graminearum, F. culmorum, A. ochraceus, and $P$. viridicatum did not negatively affected fungal biomass to a large extent. The effects of invertebrate grazing on fungi are known to be speciesspecific and density-dependent (Crowther et al. 2012). At low density, invertebrates may even stimulate fungal growth; inhibition appears when the predator population exceeds a certain density threshold. However, the specific situation still depends on the combination of fungal and invertebrate species.

Invertebrates do not prefer Aspergillus and Penicillium spp. as food (Scheu \& Simmerling, 2004; Maraun et al., 2003). The crude extract of five Penicillium strains caused weight loss and mortality of Spodoptora littorals (Paterson et al., 1987). The development of the sap beetle Carpophilus stagnated while feeding on Aspergillus flavus as much as by starvation (Wicklow et al. 1988).

Wolfarth et al. (2012) reported that the nematode Aphelenchoides saprophilus and collembolan Folsomia candida caused reduction of Fusarium culmorum biomass. Different experimental conditions and methods used to quantify fungal biomass might explain the contrasting results that were obtained in our study with $F$. culmorum. For example, Wolfarth et al. (2012) were using ELISA to quantify Fusarium protein equivalents, which might not able to take into account spores as accurately as real time PCR. In the field, the relationships might be further complicated by the effect of both partners on the plant host. For instance, Meloidogyne spp. increased the severity of Fusarium wilt in cotton roots (Hasan, 1993). Weakening plant defense or even vectoring the pathogen could have been the cause. 
Strikingly, only the biomass of $F$. verticillioides was reduced by collembolans in our studies. F. verticilliodes is reported to be dispersed by various insects (Munkvold, 2003). It promotes the growth of various invertebrates (Schulthess et al. 2001; Cardwell et al. 2000). Conversely, the same fungus was reported as an entomopathogen, causing the death of invertebrates (Pelizza et al. 2010). Fornelli et al. (2004) reported that fumonisin B1, which is a mycotoxin produced by $F$. verticillioides, show low toxicity effect to SF-9 insect cells. Many studies demonstrated that $F$. verticillioides represents a relatively attractive food source for fungal grazers (see Schulthess et al. 2001 and work referred to within).

Our research demonstrated that Folsomia candida prefers $F$. verticilliodes over $F$. graminearum, which is consistent with the observed larger biomass loss of $F$. verticilliodes than $F$. graminearum in single and mixed cultures. The finding also indicates that insects probably interact more often with $F$. verticilliodes and explains why the insect can spread F. verticillioides better compared to F. graminearum (Munkvold, 2003).

Interestingly, $F$. candida prefers the $\triangle$ AUR $F$. graminearum instead of $F$. verticillioides, which is not able to produce aurofusarin. This indicates that aurofusarin plays an important role for the host to avoid predation by small animals, and acts as an important defense agent for the fungus. The effect is similar to known antifeedants, except that aurofusarin synthesis was proved to be induced by grazing. Similar antifeedant compounds have been well-investigated in plants (Thoison et al. 2004; Simmonds, 2006). So far are only three antifeedant compounds have been detected in fungi (A. flavus) (Wicklow et al., 1988). However, the effect of these compounds on predation by fungi is uncertain.

Regarding F. graminearum strains deficient in the synthesis of deoxynivalenol, zearalenone (chapter 3), aflatoxins (unpublished), fumonisins (Sal 2018) and phosphonates (Vinas, 2018), animals were not cable of distinguishing them from their wild types. Many studies indicated that toxic secondary metabolites can protect fungi from predators (Rohlfs, 2007; 2011), but conclusive prove was missing for all proposed defense metabolites. Our studies illustrated that most of the major mycotoxins appear not play a role in deterring fungal grazers. This contradicts the hypothesis that was held for three decades (Dowd et al., 1989; Dowd et al., 1992).

Folsomia candida preferred mixed diet consisting of both $F$. verticilliodes and $F$. graminearum mycelia instead of single cultures of each fungal species. This is consistent 
with the results that were reported by Scheu \& Folger (2004). These authors suggested that feeding on mixed diet benefits collembolan through better reproduction and fitness. They argue that this might be due to diluted toxic metabolites and a more balanced nutrition. However, the underlying mechanisms are still unclear. Some Fusarium spp. have been shown to attract insects to infested kernels (Guo et al. 2014). Whether the increased preference of collembolans for mixed $F$. verticillioides and $F$. graminearum diet was due to the release of some volatile compound that attracted $F$. candida during the interaction or for other reasons needs further investigation.

$F$. candida recognized and preferred $F$. graminearum mutants that do not produce aurofusarin. Other invertebrates, the mealworms Tenebrio tomentosa and T. molitor preferred non-aurofusarin-containing diet as well ( $F$. graminearum $\triangle$ AUR and wheat). In adddition, the growth of the animals was reduced when fed on aurofusarin-containing diet (F. graminearum wild type train and wheat containing high amounts of aurofusarin). The observed growth reduction, however, could have been also caused the reduction of food intake instead of toxic effects of aurofusarin (chapter 4).

qRT-PCR and HPLC-DAD analysis showed that aurofusarin production in F. graminearum was largely induced after grazing by springtails. In addition, the red pigment induction can be clearly seen in cultures of $F$. poae, F. venenatum, $F$. sporotrichioides and F. avenaceum after exposure to springtails and the isopod Porcellio scaber. Synthesis of secondary metabolites costs energy, and fungi are expected to produce potential chemical weapons only when under pressure from fungal grazers. Thus, the induction of aurofusarin synthesis by predator feeding provides further support for the hypothesis that aurofusarin has evolved as an inducible defense compound in some Fusarium spp.

Viomellein is a dimeric naphtho- $\gamma$-pyrone pigment with chemical structure that is similar to aurofusarin. It is produced by many fungal species, including A. ochraceus. We hypothesize that this secondary metabolite possesses a similar ecological function as aurofusarin. Accordingly, after exposure of Aspergillus ochraceus to $F$. candida and $T$. molitor, HPLC-DAD analysis of the grazed mycelium showed that viomellein was induced. However, the induced amounts of viomellein in A. ochraceus were not as high as aurofusarin in F. graminearum under similar conditions. We hypothesize that this compound might be efficient in smaller amounts than aurofusarin, but this needs further 
investigation. In addition, the deterrence of small animals by viomellein has not been demonstrated yet.

Our study also showed that aurofusarin displayed no or low toxicity and deterred invertebrates only at very high concentration, for example $10 \mathrm{mg} / \mathrm{g}$. According to Steinberg's terminology, aurofusarin might function as quantitative defense agent, similar to herbivore deterrents in plants (Steinberg, 1988). Many Fusarium spp. are able to produce aurofusarin in a large quantity up to $5 \%$ of their dry weight. According to our observation, this compound is unpalatable to small animals, and thus leads to starvation if animals are not provided alternative food. Aurofusarin is a pigment of red or yellow color, depending on $\mathrm{pH}$. A common fungal pigment is melanin. This tyrosine polymer that is hard to digest and its quantity in fungal mycelia is high (dry weight in fungal mycelium up to $43.9 \%$ ) (Allam \& El-Zaher, 2012). Scheu \& Simmerling (2004) also revealed that a melanin-deficient strain of A. fumigatus is slightly more attractive to $F$. candida and $P$. armata than the wild type strain which accumulates melanin.

We also performed RNAseq analysis for $F$. graminearum IFA66 treated with $F$. candida for 48 hours and for a control culture without grazing. The results showed for the first time that, compared with $F$. graminearum control, treatment with $F$. candida results in the upregulation of pathways for various proteins and secondary metabolites, which were in general very different from chemicals known to be involved in interactions of herbivores with plants. Apart from aurofusarin, we also found that fusarin $\mathrm{C}$ and fusaristatins A have been induced by collembolan grazing. Fusarin $\mathrm{C}$ belongs to mycotoxins and can be produced by various Fusarium spp., including $F$. graminearum, $F$. moniliforme, $F$. verticillioides, $F$. fujikuroi and $F$. venenatum. Until now, no biological function of fusarin $\mathrm{C}$ is known and no report about the role of fusarin $\mathrm{C}$ in interaction with invertebrate has been published. However, the secondary metabolite NG-391 produced by Metarhizium robertsii is very similar to fusarin $\mathrm{C}$ (the only difference is a methyl group on the side chain of one of the residues of the nonribosomal peptide moiety), suggesting that both metabolites may be toxic to invertebrates. However, no role of NG-391 in the virulence of M. robertsii on Spodoptera exigua larvae could be shown (Donzelli et al. 2010).

Fusaristatin A belongs to acyl tetramic acid natural products and shows potential anticancer activity. Its biosynthesis pathway is similar to emericellamide of A. nidulans and has been well-studied (Sørensen et al., 2014). Döll et al. (2013) revealed that, after 
grazing by $F$. candida, production of emericellamide in A. nidulans was induced. This suggests that this group of compounds might contribute to defense against springtails in different fungal species.

The RNAseq analysis also pointed out induction of many small secreted cysteine-rich proteins (SSCPs), which usually function as effector proteins of plant pathogens (Qi et al., 2016). There were 197 SSCPs in F. graminearum, 34 of which were expressed during wheat infection. Among them, 15 SSCP were suggested as candidate effectors assisting in the pathogenesis of devastating disease (Fusarium head blight) of wheat (Lu \& Edwards. 2015). It is reported that effectors can suppress plant immunity (Qi et al., 2016). We enumerated 8 most up-regulated SSCP during $F$. graminearum interaction with $F$. candida (Chapter 4), which possibly play an important role in fungal defense against springtails. None of the 8 peptides induced by grazing, however, belonged to the group of effectors induced during fungal plant colonization.

Consistent with the low variation of grazing preferences by collembolans between wildtypes and mutants of Fusarium spp with present or absent production of deoxynivalenol, zearalenone and phosphonates, induction of the transcription of genes responsible for their synthesis by small animal's grazing was not detected in RNAseq data. Concurringly, Blaney et al. (1986) reported that zearalenone content in maize grains was not affected by the extent of damage of maize ears by ear worms (Heliothis sp.).

Many RNAseq data from $F$. graminearum interacting with host plants have been published (Puri et al. 2016; Hofstad et al., 2016). The RNAseq dataset for interaction with $F$. candida is the first report from an interaction with a fungal grazer and provides valuable insights into the biosynthetic pathways affected by predator grazing.

Volatile compounds are considered to be responsible for the food preference of animals in many cases (Morath et al., 2012; Bengtsson et al., 1991; Hedlund et al., 1995). Other studies demonstrated that non-volatile secondary metabolites contribute to deterring phenomena (Wicklow et al., 1988; Rohlfs et al., 2007; 2011), but as compared to VOCs these cases are rare. In our study, we found that aurofusarin, possibly together with other metabolites of the dimeric naphthopyrones in other fungal species, can contribute to the protection of fungi from predators.

In conclusion, reduction of the biomass of mycotoxin-producing fungi by collembolan grazing appears to be species-dependent. Selective feeding of collembolans on fungi is 
likely to shape the communities of mycotoxin-producing fungi in the ecosystem. Accumulation of aurofusarin may account for these differences in feeding preference because aurofusarin is a strong antifeedant, protecting fungal mycelia from predators. Regarding the other metabolites induced by feeding such as viomellein, fusarin $\mathrm{C}$, fusaristatin $\mathrm{A}$ and various proteins, further investigations are needed to elucidate their biological function.

\section{References}

Allam, N.G. and El-Zaher, E.A. (2012). Protective role of Aspergillus fumigatus melanin against ultraviolet (UV) irradiation and Bjerkandera adusta melanin as a candidate vaccine against systemic candidiasis. African Journal of Biotechnology 11: 65666577 .

Bengtsson, G., Hedlund, K. and Rundgren, S. (1991). Selective odor perception in the soil collembola Onychiurus armatus. Journal of Chemical Ecology 17: 2113-2125.

Blaney, B.J., Ramsey, M.D. and Tyler, A.L. (1986). Mycotoxins and toxigenic fungi in insect-damaged maize harvested during 1983 in far North Queensland. Crop \& Pasture Science. 37: 235-244.

Cardwell, K.F., Kling, J.G., Maziya-Dixon, B. and Bosque-Perez, N.A. (2000). Interactions between Fusarium verticillioides, Aspergillus flavus, and insect infestation in four maize genotypes in lowland Africa. Phytopathology 90: 276284.

Crowther, T.W. and Donald, A'Bear. (2012). Impacts of grazing soil fauna on decomposer fungi are species-specific and density-dependent. Fungal Ecology 5: 277-281.

Donzelli, B.G.G., Krasnoff, S.B., Churchill, A.C.L., Vandenberg, J.D. and Gibson, D.M. (2010). Identification of a hybrid PKS-NRPS required for the biosynthesis of NG391 in Metarhizium robertsii. Current Genetics 56: 151-162.

Dowd, P. F. (1992) Insect interaction with mycotoxin-producing fungi and their hosts. In: Handbook of Applied Mycology. Vol. 4. Fungal Biotechnology (D. K. Arora, R. P. Elander, K. G. Mukerji. Eds.) Marcel Dekker Inc., New York, pp 137-155 (1992).

Dowd, P. F., Miller, J. D. \& Greenhalgh, R. (1989) Toxicity and interactions of some Fusarium graminearum metabolites to caterpillars. Mycologia 81, 646-650. 
Döll, K., Chatterjee, S., Scheu, S., Karlovsky, P. and Rohlf, M. (2013). Fungal metabolic plasticity and sexual development mediate induced resistance to arthropod fungivory. Proceedings of the Royal Society B: Biological Sciences 280: 20131219.

Fornelli, F., Minervini, F. and Mule, G. (2004). Cytotoxicity induced by nivalenol, deoxynivalenol, and fumonisin B1 in the SF-9 insect cell. In Vitro Cellular \& Developmental Biology 40: 166-171.

Hasan, A. (1993). "The role of fungi in fungus-nematode interactions" In: Nematode Interactions (M. W. Khan, ed.) Springer, Dordrecht, pp 273-287.

Hedlund, L., Bengtsson, G. and Rundgren, S. (1995). Fungal odour discrimination in two sympatric species of fungivorous collembolans. Functional Ecology 9: 869-875.

Hofstad, A.N., Nussbaumer, T., Akhunov, E., Shin, S., Kugler, K.G., Kistler, H.C., Mayer, K.F.X., Muehlbauer G.Y. (2016). Examining the transcriptional response in wheat Fhb1 near-isogenic lines to Fusarium graminearum infection and deoxynivalenol treatment. The Plant Genome 9 (1).

Karlovsky, P. (1999). Biological detoxification of fungal toxins and its use in plant breeding, feed and food production. Natural Toxins 7: 1-23.

Lu, S.W. and Edwards, M.C. (2015). Genome-wide analysis of small secreted cysteinerich proteins identifies candidate effector proteins potentially involved in Fusarium graminearum-wheat interactions. Phytopathology 106: 166-176.

Maraun, M., Martens, H., Migge, S., Theenhaus, A. and Scheu, S. (2003). Adding to 'the enigma of soil animal diversity': Fungal feeders and saprophagous soil invertebrates prefere similar food substrates. European Journal of Soil Biology 39: $85-95$.

Morath, S.U., Hung, R. and Bennett, J.W. (2012). Fungal volatile organic compounds: a review with emphasis on their biotechnological potential. Fungal Biology Reviews 26: 73-83.

Munkvold, G.P. (2003). Epidemiology of Fusarium diseases and their mycotoxins in maize ears. European Journal of Plant Pathology 109: 705-713. 
Paterson, R.P.M., Simmonds, M.S.J. and Blaney W.M. (1987). Mycopesticidal effects of characterized extracts of Penicillium isolateds and purified secondary metabolites (including mycotoxins) on Drosophila melanogaster and Spodoptora littoralis. Journal of Invertebrate Pathology 50: 124-133.

Pelizza, S.A., Stenglein, S.A., Cabello, M.N., Dinolfo, M.I. and Lange, C.E. (2010). First record of Fusarium verticillioides as an entomopathogenic fungus of grasshoppers. Journal of Insect Science 11: 1-8.

Puri, K., Yan, C.H., Leng, Y.Q. and Zhong, S.B. (2016). RNA-Seq revealed differences in transcriptomes between $3 \mathrm{ADON}$ and 15ADON populations of Fusarium graminearum in vitro and in planta. Plos One 11 (10).

Qi, M., Link, T.I., Müller, M., Hirschburger, D., Pudake, R.N., Pedley, K.F., Braun, E., Voegele, R.T., Baum, T.J. and Whitham, S.A. (2016). A small cysteine-rich protein from the Asian soybean rust fungus, Phakopsora pachyrhizi, suppresses plant immunity. Plos Pathogens 12 (9).

Rohlfs, M., Albert, M., Keller, N.P. and Kempken, F. (2007). Secondary chemicals protect mould from fungivory. Biology Letters 3: 523-525.

Rohlfs, M. and Churchill, C. (2011). Fungal secondary metabolites as modulators of interactions with insects and other arthropods. Fungal Genetics and Biology 48: $23-34$.

Sal N. (2018). Einfluss von Fumonisinen auf die Interaction zwischen Fusarium verticillioides und Arthropoden. Master Thesis. Univerity of Göttingen.

Scheu, S. and Folger, M. (2004). Single and mixed diets in collembolan: effects on reproduction and stable isotope fractionation. Functional Ecology 18: 94-102.

Scheu, S. and Simmerling, F. (2004). Growth and reproduction of fungal feeding collembola as affected by fungal species, melanin and mixed diet. Oecologia 139: $347-353$.

Schulthess, F., Cardwell, F. and Gounou, S. (2001). The effect of endophytic Fusarium vertcillioides on infestation of two maize varieties by lepidopterous stemborers and coleopteran grain feeders. Phytopathology 92: 120-128. 
Simmonds, M.S.J. (2006). The search for plant-derived compounds with antifeedant activity. Advances in Phytomedicine 3: 291-324.

Steinberg, P.D. (1988). Effects of quantitative and qualitative variation in phenolic compounds on feeding in three species of marine invertebrate herbivores. Journal of Exterimental Marine Biology and Ecology 120: 221-237.

Sørensen, J.L., Sondergaard, T.E., Covarelli, L., Fuertes, P.R., Hansen, F.T., Frandsen, R.J.N., Saei, W., Lukassen, M.B., Wimmer, R., Nielsen, K.F., Gardiner, D.M. and Giese, H. (2014). Identification of biosynthetic gene clusters for the lipopeptides fusaristatin A and W493 B in Fusarium graminearum and F. pseudograminearum. Journal of Natural Products 77: 2619-2625.

Thoison, O., Sevenet, T., Niemeyer, H.M. and Russell, G.B. (2004). Insect antifeedant compounds from Nothofagus dombeyi and N. pumilio. Phytochemistry 65: 21732176 .

Vinas M. 2018. Biosynthesis and biological function of phosphonates in Fusarium spp. The biological function of phosphonates in F. oxysporum. pp 62-97. Ph.D. Thesis. University of Göttingen.

Wicklow, D.T., Dowd, P.F., Tepaske, M.R. and Gloer, J.B. (1988). Sclerotial metabolites of Aspergillus flavus toxic to a detritivorous maize insect (Carpophilus Hemipterus, Nitidulidae). Transactions of British Mycological Society 91: 433-438.

Wolfarth, F., Schrader, S., Oldenburg, E. and Weinert, J. (2013). Nematode-collembolaninteraction promotes the degradation of Fusarium biomass and deoxynivalenol according to soil texture. Soil Biology and Biochemistry 57: 903-910. 


\section{Summary and outlook}

In our study, we investigated interactions between mycotoxin-producing fungi and invertebrates. First of all, we showed that Folsomia candida grazing did not reduce fungal weight of several tested Fusarium strains except for A. nidulans. In addition, quantitative real time PCR confirmed that DNA amounts of $F$. graminearum, $F$. culmorum, $A$. ochraceus and $P$. viridicatum was not reduced by collembolan grazing. Conversely, that of $F$. verticilliodes was even significantly decreased.

After that, we checked the various aspects of food quality of $F$. graminearum and $F$. verticillioides to the collembolan Folsomia candida. We could show that $F$. candida preferred $F$. verticillioides and avoided $F$. graminearum. Experiments with mutant strains revealed that the naphthopyrone aurofusarin was the reason why collembolans preferred $F$. verticillioides to $F$. graminearum. In addition, $F$. candida preferred even more a mixture of $F$. verticillioides and $F$. graminearum to single cultures of the two fungi. This promotes further future studies of invertebrate food preference of additional mycotoxin-producing fungal species.

To confirm the defensive effect of aurofusarin, we studied food preference between $F$. graminearum and its aurofusarin deficient strains with $F$. candida, T. tomentosa and $T$. molitor. And we proved that the induction of aurofusarin qRT-PCR of the respective genes and HPLC-UV of the produced mycotoxins. Collembolan and isopod grazers induced a red pigment in the hyphae that was aurofusarin. Aspergillus ochraceus produces viomellein, a naphthopyrone with a similar structure to aurofusarin. We observed a similar effect in assays with the same animals. However, whether it functions similarly as aurofusarin, i.e. protecting the fungus by causing feeding-deterrent effects, still needs more investigations. In the future, it might be interesting to create non-producing viomellein mutant, and continue with food choice assays.

Fusarin $\mathrm{C}$ is also induced according to the RNAseq data. According to our observations, it causes no feeding deterrence effects with consecutive animal size reduction. Our hypothesis is that this compound might contribute to fungal defense against invertebrates in a subtler way. Alternatively, the negative effect might appear only after many generations. Nematodes reproduce very fast. Fitness experiment using these invertebrates might answer these questions. 
Fusaristatin A, which is related to emericellamide produced by A. nidulans, was induced by $F$. candida grazing as well. Together with the other mentioned compounds, this might be a group of mycotoxins that possess potential to defend against fungal grazers. However, the underlying mechanisms are still not clear.

In conclusion, interactions between invertebrates and fungi have been well-studied previously in an ecological context. In our study, the inclusion of mutant strains that lacked the production of specific feeding deterrent metabolites provided more robust results in terms of an unequivocal identification of defense properties of the putative metabolites. 


\section{Curriculum vitae}

Personal Information

Name: $\quad$ Yang $\mathrm{Xu}$

Place of Birth: $\quad$ Shaanxi, China

Academic Education

Oct. $2014-$ Jul. 2018

International Ph.D. Program for Agricultural Sciences in Göttingen (IPAG)

Molecular Phytopathology and Mycotoxin Research

Department of Crop Science

Georg August University Göttingen

Sep. 2011 - Jul. 2014

Master of Agriculture

Forest Protection

College of Forestry

Northwest A \& F University

Sep. 2007 - Jul. 2011

Bachelor of Engineering

Biological Engineering

College of Biological Engineering

Zhejiang Chinese Medical University 


\section{Publications \& Workshops}

\section{Publications}

Xu Y., Pfohl K. and Karlovsky P. (2016). Interaction between fungivorous Cllembola and mycotoxin-producing Fusarium species. $38^{\text {th }}$ Mycotoxin Workshop. Society for Mycotoxin Research. Berlin - Germany. Book of Abstracts: pp. 157.

Xu Y., Nan X.N., Wei C., He H. (2016). Seasonal characteristics of gut bacterial communities associated with carpenter ant Camponotus japonicus (Hymenoptera: Formicidae). Acta Entimologica Sinica 59: 632-640

Xu Y., Pfohl K. and Karlovsky P. (2015). Protection of fungivorous collembola against poisioning by mycotoxins. $37^{\text {th }}$ Mycotoxin Workshop. Society for Mycotoxin Research. Bratislava-Slovakia. Book of Abstracts: pp. 157.

Xu Y., Ge L.J., Zhu Z.H. (2010). Practice and reform on teaching biotechnology. Research Progress of Higher Medical Education in China 1: 134-136. (In Chinese) 


\section{Workshops \& Conferences}

01 - 03 June 2015

$37^{\text {th }}$ Mycotoxin Workshop. Society for Mycotoxin Research, Bratislava - Slovakia.

$\underline{\text { Poster Presentation }}$

02-04 May 2016

$38^{\text {th }}$ Mycotoxin Workshop. Society for Mycotoxin Research, Berlin - Germany.

$\underline{\text { Poster Presentation }}$ 


\section{Acknowledgment}

My best compliments and sincere gratitude goes to my supervisor Prof. Dr. Petr Karlovsky-Georg August University Göttingen. Thank him for offering me opportunity to study in this great university and providing me this interesting research project. I really appreciate his invaluable academic ideas, experimental support, unconditional care and trust. I would never forget this wonderful mentorship, which supports me to finish my $\mathrm{PhD}$ work and build up my scientific confidence.

I would like to than Dr. Katharina Pfohl, Dr. Franz Hadaceck and Dr. Anna Rathgeb for introducing me HPLC knowledge and usage, and all their valuable support, suggestions. I am thankful to Ruth Pilot and Heike Rollwage for their constant technical support and unconditional care. My gratitude particularly goes to Ruth Pilot for her involvement of my mealworms food choice experiment.

I acknowledge Maria Vinas, Albatol Alsarrag for helping me finish my project with RNAseq, HPLC-DAD, HPLC-MS analysis. And I am very grateful to Vicent Ayugi, who helped me to check my thesis carefully. I also really appreciate their valuable friendship.

A lot of thanks to my scholarship CSC (China schplarship council) for offering me financial support to study here, which assistant me finishe my $\mathrm{PhD}$ degree and opens my mind a lot in Europe.

Many thanks to all my friend in our Lab: Rasoul Abousaeedi, Riyan Anggriawan, Elika, Zhqing Guo, Monhammad Sherif, Minhammad Alhussein, Shushe Zhang, Nuo Jin, Viviana, Ling Su, Wanying He, Rosine, Pervin, Lukas, Simon, Katharina Henze, Isabella Reutz, Jia Lai, Zana Jamal Kareem, Zahaar, Makus, Aulia, Nilay and Jessica, for the nice environment and pleasure moment we spent together. And special thanks to my friend Xuying Zhang, we spend a lot of spare time together, I really appreciate the nice food, beautiful scenery enjoyed together with her.

I would like express my deepest thank to my family and my fiancé Lei $\mathrm{Xu}$, for their diverse support and love they give me for so many years... 


\section{Statutory declaration}

1. I, hereby, declare that this Ph.D. dissertation has not been presented to any other examining body either in its present or a similar form.

Furthermore, I also affirm that I have not applied for a Ph.D. at any other higher school of education.

Gottingen,

2. I, hereby, solemnly declare that this dissertation was undertaken independently and without any unauthorized aid.

Gottingen, 